University of Tennessee Health Science Center

UTHSC Digital Commons

\title{
Sleep, Fatigue and Caregiver Burden in Parents of Children with Acute Lymphoblastic Leukemia (ALL)
}

Jerithea Tidwell

University of Tennessee Health Science Center

Follow this and additional works at: https://dc.uthsc.edu/dissertations

Part of the Neoplasms Commons, and the Psychiatric and Mental Health Commons

\section{Recommended Citation}

Tidwell, Jerithea, "Sleep, Fatigue and Caregiver Burden in Parents of Children with Acute Lymphoblastic Leukemia (ALL)" (2008). Theses and Dissertations (ETD). Paper 274. http://dx.doi.org/10.21007/ etd.cghs.2008.0319.

This Dissertation is brought to you for free and open access by the College of Graduate Health Sciences at UTHSC Digital Commons. It has been accepted for inclusion in Theses and Dissertations (ETD) by an authorized administrator of UTHSC Digital Commons. For more information, please contact jwelch30@uthsc.edu. 


\title{
Sleep, Fatigue and Caregiver Burden in Parents of Children with Acute Lymphoblastic Leukemia (ALL)
}

\begin{abstract}
Parents often report that their child diagnosed with acute lymphoblastic leukemia (ALL) and receiving steroid therapy experience altered sleep patterns, fatigue, irritability, and other behavioral changes. As a consequence, parents may experience altered sleep, fatigue, and increased caregiver burden. If so, the differences seen in parental sleep efficiency and sleep duration may be attributed to the child experiencing altered sleep secondary to receiving dexamethasone. The purpose of this study was to describe, caregiver sleep, fatigue, and caregiver burden immediately before and during their child's dexamethasone pulse in continuation therapy for ALL and to compare parent quality of sleep and fatigue with their child's sleep. This descriptive comparative study is a companion study to the Sleep, Fatigue, and Dexamethasone in Childhood ALL protocol (SLEEP) and the St Jude Children's Research Hospital Total XV protocol for children with ALL. Research participants were parent(s) or guardian(s) of children enrolled on Total XV (in the treatment phase of continuation) and enrolled on SLEEP. Both mothers and fathers were eligible to participate. Research participants were asked to complete a demographic sheet, a fatigue measure, and caregiver burden instruments. Research participants were also asked to keep a sleep diary and to wear a wrist actigraph to measure sleep quality. Parent sleep quality, level of fatigue, and caregiver burden were measured and compared during two consecutive 5-day periods, (the first when their child was off dexamethasone and the second 5-day period when the child was on dexamethasone). Fatigue and caregiver burden of the participants were compared between the two time periods. Sleep quality of the parents was compared between the two time periods and compared with the sleep quality of their ill child during the same consecutive 5-day periods.

Data were analyzed using descriptive statistics, one sample $t$-test, and mixed effects models. The sample consisted of twenty-two parents, of which $77.3 \%$ were primary caregivers ( 15 mothers and 2 fathers). The majority of primary caregivers were white, female, and homemakers at the time of the study. Their average age was 38 years $(S D=6)$. These caregivers had higher sleep efficiency and sleep duration, measured by actigraphy, when their child was on dexamethasone compared to when their child was off dexamethasone. Primary caregivers had a significantly lower actual sleep time and sleep duration compared to child's actual sleep time and sleep duration. Actual sleep time for parent caregivers and their children were significantly higher when on dexamethasone $(p=.002)$

Primary caregivers reported low fatigue scores. However, higher fatigue scores were reported when the children were on dexamethasone as compared to when the children were off dexamethasone. Subjective burden scores for parents were significantly higher in week 2 (on dexamethasone) than in week 1 (off dexamethasone $(p=0.05)$. Average scores for objective burden were slightly higher during week 2 as compared to week 1 . Further research is needed to explore differences in caregiver burden and fatigue by parent sex, marital status, ethnicity, and child's phase of treatment.
\end{abstract}

\section{Document Type}

Dissertation

\section{Degree Name}

Doctor of Philosophy (PhD)

\section{Program}

Nursing

\section{Research Advisor}

Pamela S. Hinds RN, Ph.D. 


\section{Keywords}

Sleep, Caregiver Burden, Fatigue, ALL, Acute Lymphoblastic Leukemia

\section{Subject Categories}

Diseases | Medicine and Health Sciences | Mental and Social Health | Neoplasms | Psychiatric and Mental Health 


\title{
SLEEP, FATIGUE, AND CAREGIVER BURDEN IN PARENTS OF CHILDREN
} WITH ACUTE LYMPHOBLASTIC LEUKEMIA (ALL)

\author{
A Dissertation \\ Presented for \\ The Graduate Studies Council \\ The University of Tennessee \\ Health Science Center \\ In Partial Fulfillment \\ Of the Requirements for the Degree \\ Doctor of Philosophy \\ From The University of Tennessee
}

By

Jerithea Doronice Tidwell

May 2008 
Copyright (C) 2008 by Jerithea D. Tidwell All rights reserved 


\section{DEDICATION}

This dissertation is dedicated to the following people:

my awesome parents, Mr. and Mrs. Goodlow

my husband, Jose and my beautiful children, Deanna, Jordan, Jamaria and Jeremiah, my brother Tye Goodlow

my mother-in-law, Irene Tidwell

my special friends Catherine Boyce, Dr. Kim Broyles, Linda Watts-Parker, Lois Bolden and Rose Stemmons

for unconditional love and support. You inspire me. 


\section{ACKNOWLEDGEMENTS}

I would like to thank God for giving me the strength and perseverance to complete this monumental endeavor. Additionally, I would like to express my sincere gratitude to my mentors, Dr. Pamela Hinds and Dr. Mona Wicks for believing in me and providing professional and personal guidance. I would like to gratefully acknowledge the other members of my dissertation committee: Dr. Judy Martin, Dr. Deo Kumar Srivastava, Dr. David Van Brunt; consultants, Mr. James Okuma, and Dr. Ann Berger; co-investigators, Cheryl Fernadez, and Monica Ash and the magnificent staff in the Division of Nursing Research at St. Jude Children's Research Hospital for their professional insight, dedication, and support. Special thanks to Nikole Daniels for working with me through the night.

I would also like to express deep gratitude to my family, close friends, and colleagues at St. Jude Children's Research Hospital for their encouragement.

Funding for this research was funded by the Minority Supplement to NINR RO1 NR07610. 


\begin{abstract}
Parents often report that their child diagnosed with acute lymphoblastic leukemia (ALL) and receiving steroid therapy experience altered sleep patterns, fatigue, irritability, and other behavioral changes. As a consequence, parents may experience altered sleep, fatigue, and increased caregiver burden. If so, the differences seen in parental sleep efficiency and sleep duration may be attributed to the child experiencing altered sleep secondary to receiving dexamethasone. The purpose of this study was to describe, caregiver sleep, fatigue, and caregiver burden immediately before and during their child's dexamethasone pulse in continuation therapy for ALL and to compare parent quality of sleep and fatigue with their child's sleep. This descriptive comparative study is a companion study to the Sleep, Fatigue, and Dexamethasone in Childhood ALL protocol (SLEEP) and the St Jude Children's Research Hospital Total XV protocol for children with ALL. Research participants were parent(s) or guardian(s) of children enrolled on Total XV (in the treatment phase of continuation) and enrolled on SLEEP. Both mothers and fathers were eligible to participate. Research participants were asked to complete a demographic sheet, a fatigue measure, and caregiver burden instruments. Research participants were also asked to keep a sleep diary and to wear a wrist actigraph to measure sleep quality. Parent sleep quality, level of fatigue, and caregiver burden were measured and compared during two consecutive 5-day periods, (the first when their child was off dexamethasone and the second 5-day period when the child was on dexamethasone). Fatigue and caregiver burden of the participants were compared between the two time periods. Sleep quality of the parents was compared between the two time periods and compared with the sleep quality of their ill child during the same consecutive 5-day periods.
\end{abstract}

Data were analyzed using descriptive statistics, one sample $t$-test, and mixed effects models. The sample consisted of twenty-two parents, of which $77.3 \%$ were primary caregivers (15 mothers and 2 fathers). The majority of primary caregivers were white, female, and homemakers at the time of the study. Their average age was 38 years $(\mathrm{SD}=6)$. These caregivers had higher sleep efficiency and sleep duration, measured by actigraphy, when their child was on dexamethasone compared to when their child was off dexamethasone. Primary caregivers had a significantly lower actual sleep time and sleep duration compared to child's actual sleep time and sleep duration. Actual sleep time for parent caregivers and their children were significantly higher when on dexamethasone $(p=.002)$

Primary caregivers reported low fatigue scores. However, higher fatigue scores were reported when the children were on dexamethasone as compared to when the children were off dexamethasone. Subjective burden scores for parents were significantly higher in week 2 (on dexamethasone) than in week 1 (off dexamethasone $(p=0.05)$. Average scores for objective burden were slightly higher during week 2 as compared to week 1. Further research is needed to explore differences in caregiver burden and fatigue by parent sex, marital status, ethnicity, and child's phase of treatment. 


\section{TABLE OF CONTENTS}

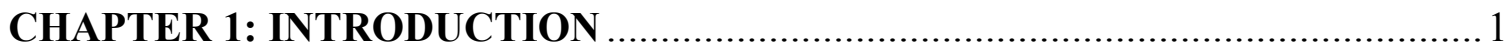

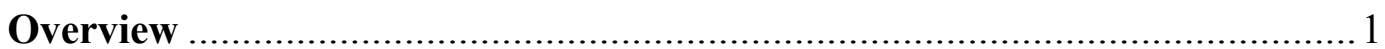

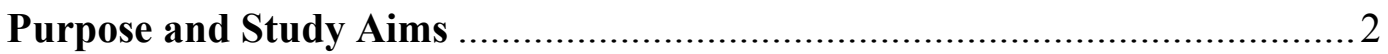

Specific Aim One - Parent Sleep..........................................................

Specific Aim Two - Parent and Child Sleep ..............................................

Specific Aim Three - Parent Fatigue .......................................................

Specific Aim Four - Parent Caregiver Burden.......................................... 3

Specific Aim Five - Relationships among Sleep, Fatigue, and

Caregiver Burden ............................................................................... 3

Specific Aim Six - Parent Sleep, Fatigue, Caregiver Burden, and

Demographic Variables .......................................................................... 3

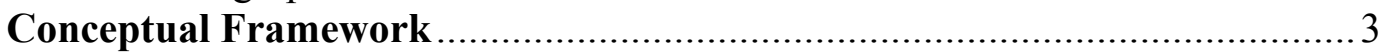

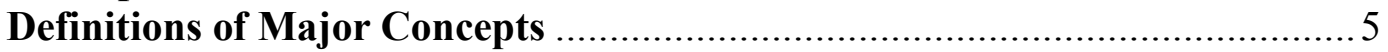

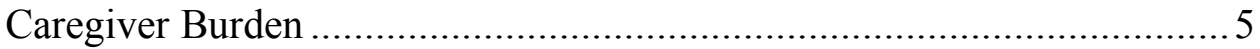

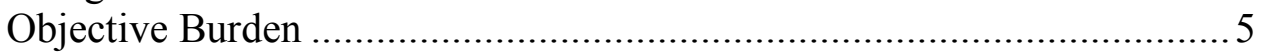

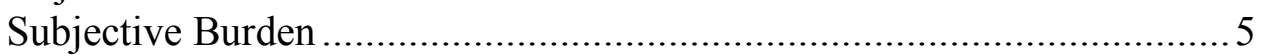

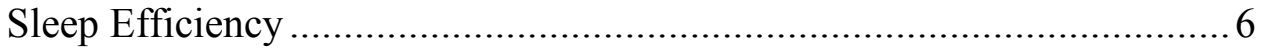

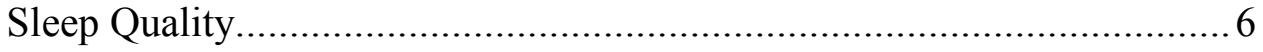

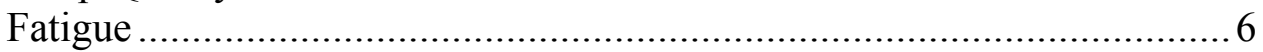

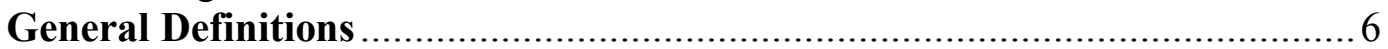

Acute Lymphoblastic Leukemia ........................................................ 6

Parental Caregivers …………………............................................

Primary Caregiver Burden..............................................................

Secondary Caregiving Burden .............................................................

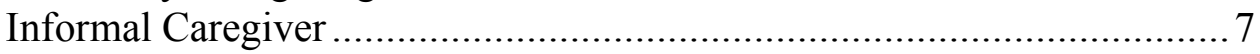

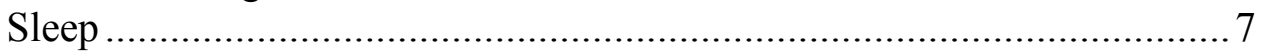

Actual Sleep Time..........................................................................

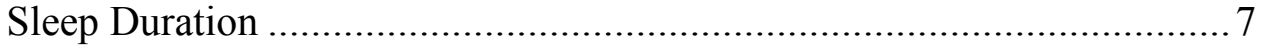

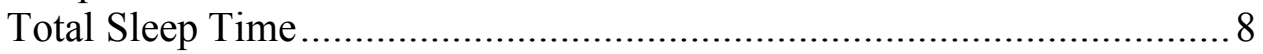

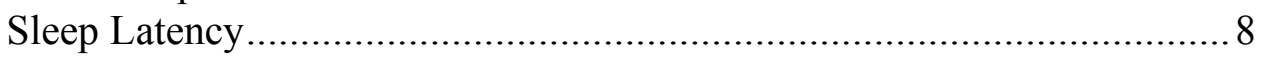

Wake after Sleep Onset .................................................................... 8

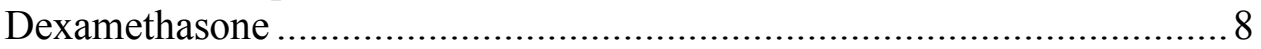

Background on Sleep, Fatigue, and Dexamethasone in Childhood ALL

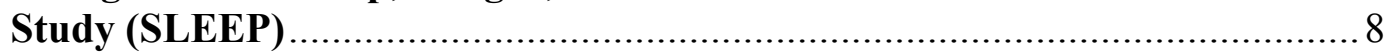

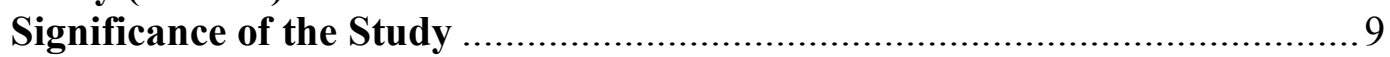

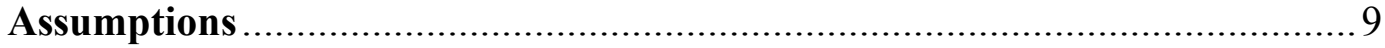

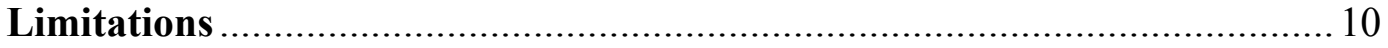

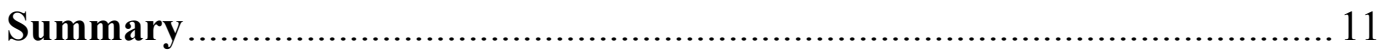

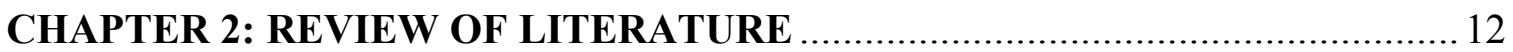

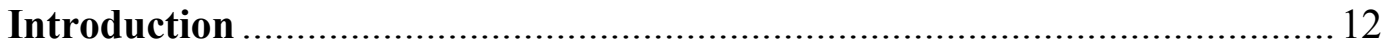

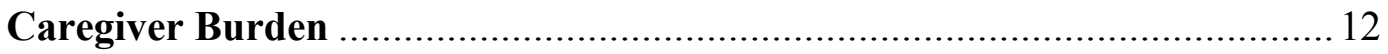


Parental Caregivers of Chronically Ill Children ................................. 13

Parental Caregivers of Children with Cancer ............................................ 16

Gender Differences in Parental Caregivers ................................................. 17

Mothers............................................................................... 18

Fathers .............................................................................. 20

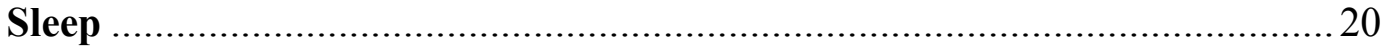

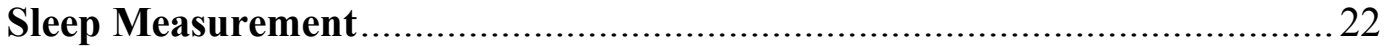

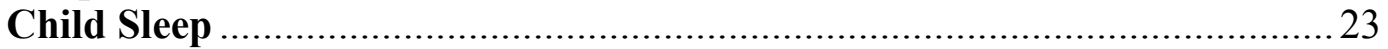

Parent and Child Sleep ..................................................................... 23

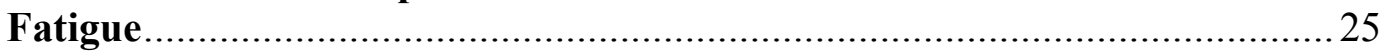

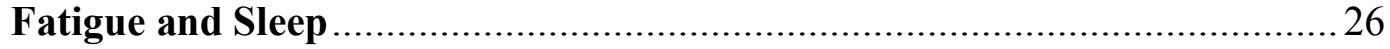

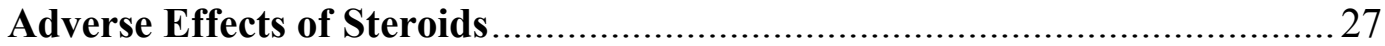

Summary of Review of Literature ................................................. 28

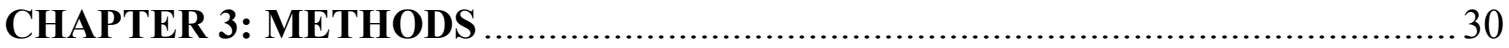

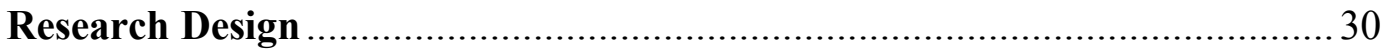

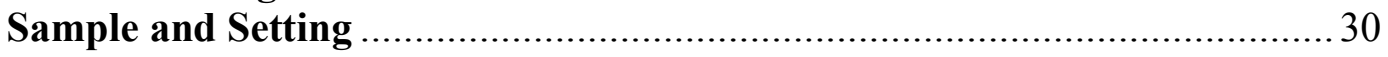

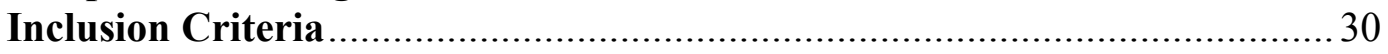

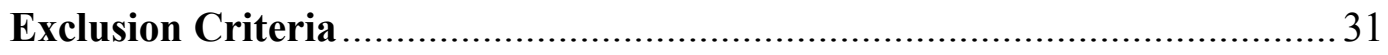

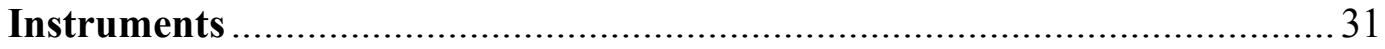

Demographic Form ..................................................................... 31

Care of My Child with Cancer Instrument ....................................... 31

Revised Informal Caregiver Survey for Parents (RICS) ...................... 32

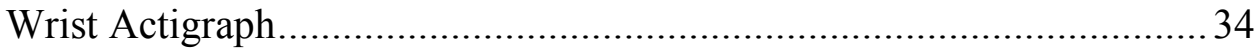

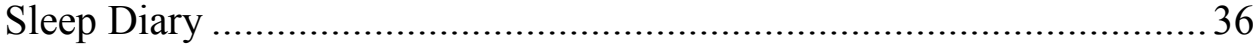

Brief Fatigue Inventory (BFI) ................................................. 36

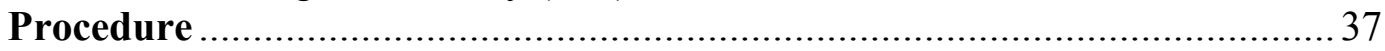

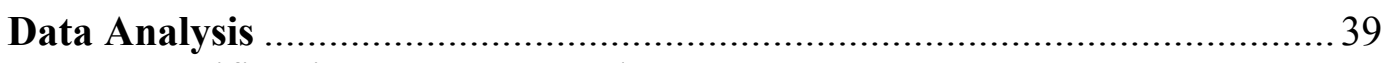

Specific Aim One - Parent Sleep.................................................. 39

Specific Aim Two - Parent and Child Sleep ....................................... 40

Specific Aim Three - Parent Fatigue ........................................... 40

Specific Aim Four - Parent Caregiver Burden ................................. 41

Specific Aim Five - Relationships among Sleep, Fatigue, and

Caregiver Burden ...................................................................... 41

Specific Aim Six - Parent Sleep, Fatigue, Caregiver Burden, and

Demographic Variables .......................................................... 42

Human Subjects Consideration ..................................................... 42

Obtaining Informed Consent .................................................... 42

Staff Training and Integrity of Intervention .................................... 42

Criteria for Removal from Protocol and Off-Study Criteria........................ 42

Safety and Adverse Event Reporting Requirements ............................... 43

Data Collection and Confidentiality ................................................. 43 


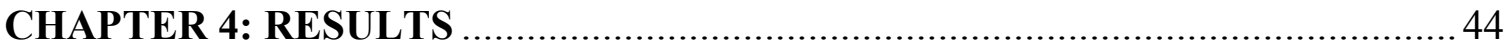

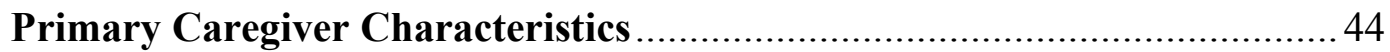

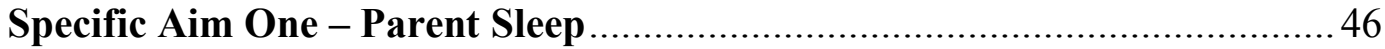

Findings for Primary Caregivers........................................................... 46

Actigraph Results for Primary Caregivers ....................................46

Sleep Diary Results for Primary Caregivers ................................. 49

Findings for Mothers .........................................................................5 54

Actigraph Results for Mothers ………………………............. 54

Sleep Diary Results for Mothers ................................................54

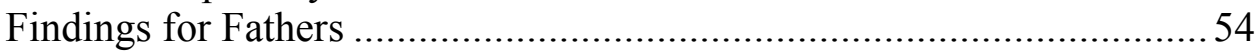

Actigraph Results for Fathers...................................................5 54

Sleep Diary Results for Fathers................................................5 55

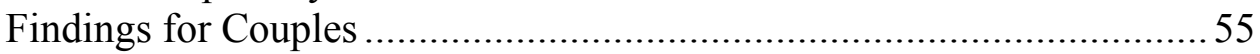

Actigraph Results for Couples .................................................... 55

Sleep Diary Results for Couples..................................................55

Specific Aim Two - Parent and Child Sleep …………................................55

Findings for Primary Caregivers and Child Sleep Variables ....................56

Findings for Mothers and Child Sleep Variables......................................58

Findings for Fathers and Child Sleep Variables .......................................58

Findings for Couples and Child Sleep Variables........................................60 60

Specific Aim Three - Parent Fatigue ..........................................................6 60

Findings for Brief Fatigue Inventory Results for Primary Caregivers...... 60

Findings for Brief Fatigue Inventory Results for Mothers .........................60 60

Findings for Brief Fatigue Inventory Results for Fathers ..........................62 62

Findings for Brief Fatigue Inventory Results for Couples ........................62 62

Specific Aim Four - Parent Caregiver Burden.............................................6. 62

Findings for Primary Caregivers..........................................................6 62

Revised Informal Caregiver Survey Results for Primary

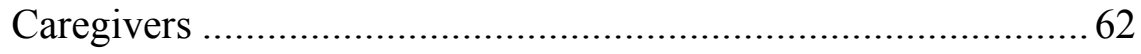

The Care of My Child with Cancer Instrument Results for

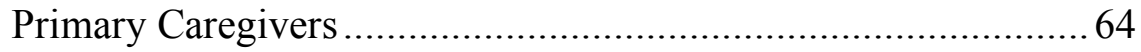

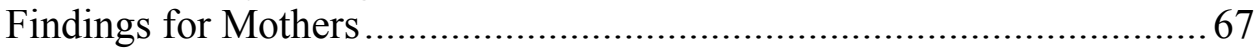

Revised Informal Caregiver Survey Results for Mothers............. 67

Care of My Child with Cancer Instrument Results for

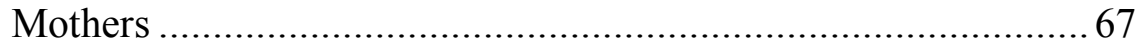

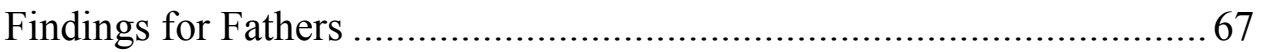

Revised Informal Caregiver Survey Results for Fathers ...............67 67

Care of My Child with Cancer Instrument Results for Fathers..... 68

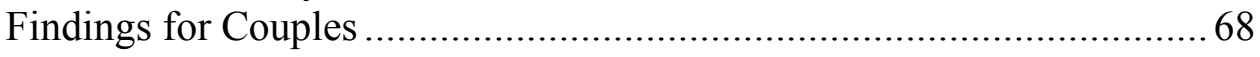

Revised Informal Caregiver Survey Results for Couples ..............6 68

Care of My Child with Cancer Instrument Results for

Couples ..... .68

Specific Aim Five - Relationships among Sleep, Fatigue, and Caregiver

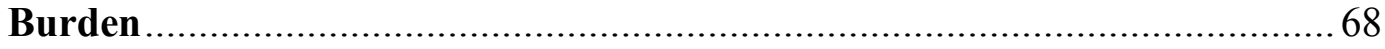

Specific Aim Six - Parent Sleep, Fatigue, Caregiver Burden, and

Demographic Variables 
Summary

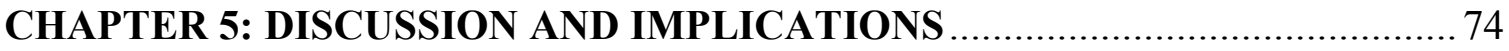

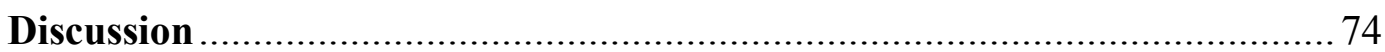

Parent Caregiver Characteristics..................................................... 74

Aim One - Parent Sleep ................................................................... 75

Aim Two - Parent and Child Sleep................................................... 77

Aim Three - Parent Fatigue............................................................... 79

Aim Four - Parent Caregiver Burden ............................................. 79

Aim Five - Relationships among Sleep Fatigue, and Caregiver Burden.. 81

Aim Six - Parent Sleep, Fatigue, Caregiver Burden, and Demographic

Variables

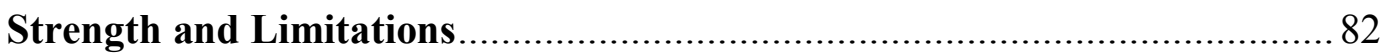

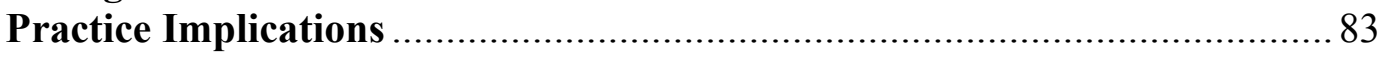

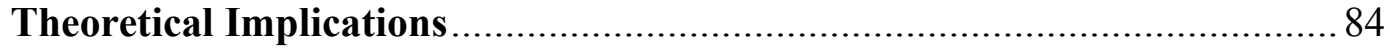

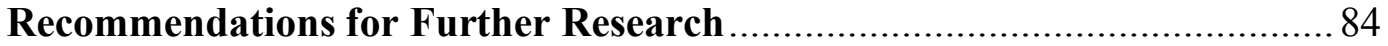

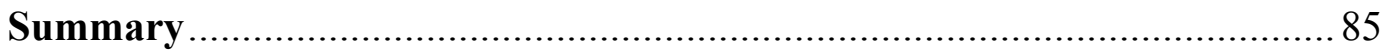

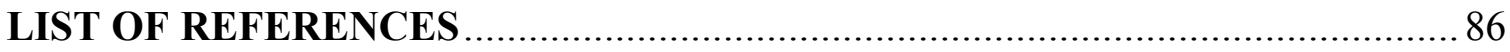

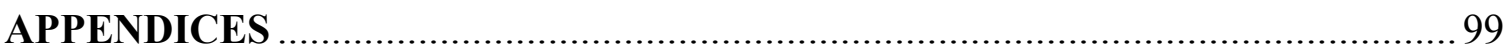

APPENDIX A. THE CARE OF MY CHILD WITH CANCER INSTRUMENT (CMCC)........................................ 100

APPENDIX B. REVISED INFORMAL CAREGIVERS SURVEY FOR

PARENTS (RICS) .............................................. 109

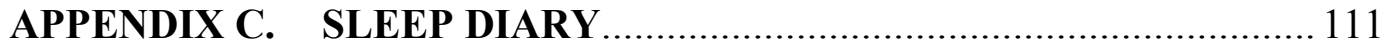

APPENDIX D. BRIEF FATIGUE INVENTORY (BFI) ..................... 116

APPENDIX E PARENT DEMOGRAPHIC FORM......................... 118

APPENDIX F. FEASIBILITY STUDY LETTER .............................. 125

APPENDIX G. FEASIBILITY STUDY DEMOGRAPHIC FORM........ 127

APPENDIX H. FEASIBILITY RELEVANT FORM ........................ 130

APPENDIX I. FEASIBILITY EASY TO UNDERSTAND FORM ........ 134

APPENDIX J. INSTRUCTIONS FOR SLEEP DIARY ........................ 137

APPENDIX K. SAMPLE PARENT PARTICIPANT LETTER .............. 140

APPENDIX L. INSTRUCTIONS FOR COMPLETING WRITTEN

FORMS ….................................................... 142

APPENDIX M. DATA COLLECTION TIME LINE .......................... 144

APPENDIX N. INFORMED CONSENT STATEMENT ….................... 146

APPENDIX O. INSTITUTIONAL REVIEW BOARD RESPONSE ....... 154

APPENDIX P. OUTLINE OF PROPOSED TRAINING WORKSHOP. 160

APPENDIX Q. SLEEP QUALITY, FATIGUE AND CAREGIVER

BURDEN STATISTICAL RESULTS FOR

MOTHERS. 
APPENDIX R. SLEEP QUALITY, FATIGUE, AND CAREGIVER BURDEN STATISTICAL RESULTS FOR

FATHERS

APPENDIX S. SLEEP QUALITY, FATIGUE, AND CAREGIVER BURDEN STATISTICAL RESULTS FOR

COUPLES

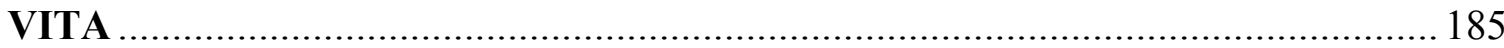




\section{LIST OF TABLES}

Table 3-1. Internal Consistency Estimates for Physical Care (PC) of the Care of My Child with Cancer Instrument............................................................ 33

Table 3-2. Internal Consistency Estimates for Emotional Care (EC) of the Care of My Child with Cancer Instrument ....................................................... 33

Table 3-3. Internal Consistency Estimates for the Revised Informal Caregiver Survey for Parents.

Table 3-4. Internal Consistency Estimates for Brief Fatigue Inventory

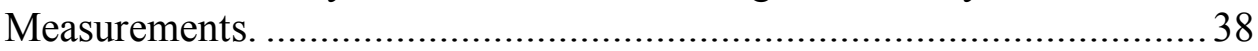

Table 4-1. Demographic Information for Primary Caregivers $(\mathrm{n}=17) \ldots \ldots \ldots \ldots \ldots \ldots \ldots \ldots . . .45$

Table 4-2. Descriptive Sleep Quality Results by Actigraph for Primary Caregivers $(\mathrm{n}=15)$.

Table 4-3. Differences in Average Sleep Quality Results between Week 2 and

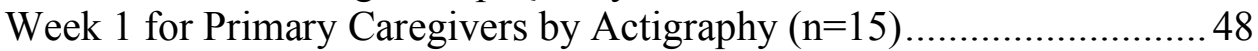

Table 4-4. Summary of Longitudinal Proc Mixed Regression Model Findings for the Primary Caregivers' Actigraphy Scores.

Table 4-5. Sleep Duration Scores by Actigraph and Sleep Diary Report for Primary Caregivers.

Table 4-6. Difference in Sleep Duration Results between Actigraph and Sleep Diary Reports for Primary Caregivers......................................................51

Table 4-7. Summary of Sleep Diary Results for Primary Caregivers........................52

Table 4-8. Summary of Subjective Sleep Quality for Primary Caregivers.................53

Table 4-9. $\quad$ Sleep Quality Results for Matched Primary Caregiver and Child by Actigraphy $(\mathrm{n}=14)$.

Table 4-10. Summary of Proc Mixed Model Regression Findings for Matched Primary Caregiver and Child

Table 4-11. Descriptive Brief Fatigue Inventory Results for Primary Caregivers ....... 61

Table 4-12. Descriptive Revised Informal Caregiver Survey Results for Primary Caregivers $(\mathrm{n}=16)$ 
Table 4-13. Descriptive Care of My Child with Cancer Instrument Results for Primary Caregivers $(\mathrm{n}=14)$.

Table 4-14. Differences in Care of My Child with Cancer Instrument Results for Primary Caregivers.

Table 4-15. Summary of Proc Mixed Model Regression Findings Related to Sleep, Fatigue, and Caregiver Burden within each of the 5-day Periods

Table 4-16. Results for Univariate Regression Analysis Assessing Relationship between Sleep Quality, Fatigue, Caregiver Burden, Parent's Age, Ethnicity, Marital Status, Child's Risk Category, and Child's Age

Table Q-1. Descriptive Sleep Quality Results by Actigraph for Mothers (n=13).... 164

Table Q-2 Differences in Average Sleep Quality Results by Week 1 and Week 2 for Mothers by Actigraph ( $\mathrm{n}=13)$.

Table Q-3. $\quad$ Summary of Longitudinal Proc Mixed Regression Model Findings for Mothers' Actigraphy Scores.

Table Q-4. Sleep Duration Results by Actigraph and Sleep Diary Report for Mothers. 166

Table Q-5. Sleep Quality Results for Matched Mother and Child Sleep by Actigraphy ( $\mathrm{n}=12)$.

Table Q-6. Summary of Proc Mixed Model Regression Findings for Mother and Child.

Table Q-7. Descriptive Brief Fatigue Inventory Results for Mothers 168

Table Q-8. Differences in Average Brief Fatigue Inventory Results between Week 1 and Week 2 for Mothers

Table Q-9. Descriptive Revised Informal Caregiver Survey Results for Mothers $(\mathrm{n}=14)$

Table Q-10. Descriptive Care of My Child with Cancer Instrument Results for Mothers $(\mathrm{n}=12)$

Table Q-11. Differences in Care of My Child with Cancer Instrument Results by Week 1 and Week 2 for Mothers 
Table R-1. Descriptive Sleep Quality Results by Actigraphy for Fathers $(n=5) \ldots \ldots .173$

Table R-2. Sleep Duration Results by Actigraph and Sleep Diary Report for

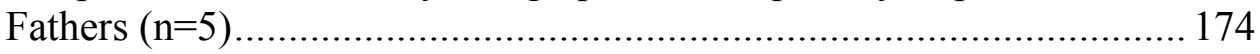

Table R-3. Difference in Sleep Duration Results between Actigraph and Sleep

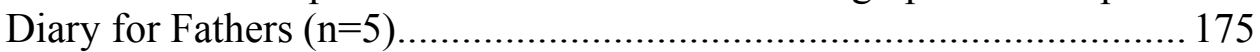

Table R-4. Sleep Quality Results for Matched Father and Child by Actigraphy $(n=4)$ 176

Table R-5. Descriptive Brief Fatigue Inventory Results for Fathers..................... 177

Table R-6. Descriptive Revised Informal Caregiver Survey Results for Fathers .... 177

Table R-7. Descriptive Care of My Child with Cancer Instrument Results for

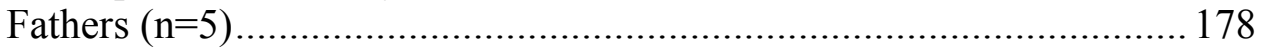

Table S-1. Descriptive Sleep Quality Results by Actigraphy for Couples $(n=4) \ldots . .180$

Table S-2. Sleep Duration Results by Actigraph and Sleep Diary Report for Couples $(n=4)$

Table S-3 Sleep Quality Results for Matched Couples and Child $(n=4) \ldots \ldots \ldots \ldots . . . .182$

Table S-4. Descriptive Brief Fatigue Inventory Results for Couples $(n=4) \ldots \ldots \ldots \ldots . .183$

Table S-5. Descriptive Revised Informal Caregiver Survey Results for Couples $(\mathrm{n}=4)$.

Table S-6. Descriptive Care of My Child with Cancer Results for Couples (n=4) .. 184 


\section{CHAPTER 1: INTRODUCTION}

\section{Overview}

Acute lymphoblastic leukemia (ALL) is the most common childhood cancer. Approximately 4000 cases of ALL are diagnosed each year in the United States. Since the 1990s, the five-year event-free survival rates for children with ALL has increased to approximately $90 \%$ (Pui \& Evan, 2007). ALL is considered a chronic illness that requires treatment, assessment, and management of complications for many years with much of the treatment being done at home.

The trend of earlier discharge and outpatient treatment of cancer means that the family is increasingly responsible for medication administration and the management of symptoms and side effects at home. Information needs for transitioning patients from hospital to home are extensive. Information ranges from concrete (flushing central line catheter) to complex (cancer treatment options) (McCorkle et al., 2000). A study was conducted by Yates (1999) exploring the strains associated with caregiving using 105 families of adult cancer patients. She found that caregivers often report feeling that they did not understand the nature of the patient's illness due to lack of information from healthcare professionals, and therefore were not doing what should be done to help the patient with cancer. Another study by Bath (2000) involving 109 partners of breast cancer patients indicated that the partner's information needs were extensive, yet health care providers often did not address these needs. Care of the patient includes care of the caregiver as well. Proper attention to the needs of the caregiver is essential to the care and health outcome of the patient and potentially for those of the family unit.

Parental health can influence and be influenced by the health of the child (Rahman et al., 2002). This reciprocal relationship is particularly well-documented in psychosocial research. An example is the documented negative influence of maternal depression on child development and on the child's overall sense of well-being, and in turn, on the maternal sense of parental competence (Rahman et al., 2002; Black et al., 2002). Although the reciprocal nature of this relationship is infrequently studied, a parent's sensitivity to his or her child's behavioral and health patterns could be the first source of warning that an ill child is affected by medication in a non-therapeutic manner. Parents may be the first to notice a change in an ill child's sleep pattern and level of fatigue. A prolonged alteration in the child's sleep pattern could influence the parent sleep as well. Parents often report that their child diagnosed with ALL and receiving a steroid therapy experiences altered quality of sleep such as being unable to sleep, delays in falling asleep, or sleeping only sporadically (Harris et al., 1986). As a consequence, parents' sleep is disrupted and fatigue can result. Because of child behavior changes during the dexamethasone period that includes decreased cooperation with health care procedures such as taking medications, parental caregiver burden tends to be higher during the dexamethasone period than during non-steroid treatment periods. The parental caregiver burden is already high for the primary caregiver of a child with cancer (Keegan-Wells et al., 2002), but is likely made more difficult when the parent is 
experiencing altered sleep and greater fatigue. Although parents have reported anecdotally these disruptions in their sleep and the attributed increases in fatigue and caregiver burden related to these disruptions, the actual changes have not been measured.

\section{Purpose and Study Aims}

The primary purpose of this study was to evaluate caregiver sleep, fatigue, and caregiver burden immediately before and during their child's dexamethasone pulse in continuation therapy for ALL and to compare parent quality of sleep and fatigue with their child's sleep. This study had the following specific aims:

\section{$\underline{\text { Specific Aim One - Parent Sleep }}$}

To compare parent sleep quality (sleep efficiency, actual sleep time, sleep duration, nocturnal awakenings and wake after sleep onset score (WASO)) during two consecutive 5-day periods (when their child being treated for ALL is off versus on dexamethasone) as measured by wrist actigraph (primary indicator) and a sleep diary (self-report measure).

Related hypothesis one: Average sleep quality scores for parental sleep before dexamethasone (week 1) will be better (greater sleep time and sleep duration, less nocturnal awakenings, sleep efficiency and WASO) than average sleep quality during dexamethasone (week 2).

\section{$\underline{\text { Specific Aim Two - Parent and Child Sleep }}$}

To assess the relationship between the parent and child sleep quality (sleep efficiency, actual sleep time, sleep duration, nocturnal awakenings, and wake after sleep onset score (WASO)) during two consecutive 5-day periods off (week 1) versus on (week 2) dexamethasone as measured by wrist actigraph.

Related hypothesis two: Average sleep quality scores (sleep efficiency, actual sleep time, sleep duration, nocturnal awakenings, and wake after sleep onset score (WASO)) for parental and child sleep before dexamethasone (week 1) will be better (greater sleep time and sleep duration, less nocturnal awakenings, sleep efficiency and WASO) than average sleep quality scores during dexamethasone (week 2). Parental and child sleep scores will have a directional relationship to one another.

\section{$\underline{\text { Specific Aim Three - Parent Fatigue }}$}

To compare parent fatigue as measured by the Brief Fatigue Inventory during the two consecutive 5-day periods of their child being off versus on dexamethasone. 
Related hypothesis three: Parent fatigue scores will be less before dexamethasone (week 1) than during dexamethasone (week 2).

\section{$\underline{\text { Specific Aim Four - Parent Caregiver Burden }}$}

To compare parental caregiver burden (time, effort, strain and difficulty of care responsibilities) during the two consecutive 5-day periods as measured by the Revised Caregiver Survey and the Care of My Child with Cancer Instruments.

Related hypothesis four: Parental subjective and objective burden scores will be greater during dexamethasone (week 2) than before dexamethasone (week 1).

\section{$\underline{\text { Specific Aim Five - Relationships among Sleep, Fatigue, and Caregiver Burden }}$}

To assess the relationships among parent sleep quality, fatigue, and caregiver burden within each of the 5-day periods.

Related hypothesis five: The relationship between parental sleep would be inversely related to parent fatigue and parent caregiver burden. Average parental scores for sleep quality (sleep efficiency, actual sleep time, sleep duration, nocturnal awakenings, and wake after sleep onset score (WASO)), fatigue, and caregiver burden before dexamethasone (week 1) will be less than average scores during dexamethasone (week 2).

$\underline{\text { Specific Aim Six - Parent Sleep, Fatigue, Caregiver Burden, and Demographic Variables }}$

To explore if parent sleep quality, fatigue, and caregiver burden differs by parent age, marital status, ethnicity, child's risk category (low versus standard) or the child's age.

\section{Conceptual Framework}

The conceptual framework guiding this study and that of the SLEEP study is the Human Response Model (Figure 1-1). The Human Response Model (HRM) integrates bio-psychological factors, individual characteristics or "person factors" that may or may not be modifiable, environmental factors that represent potential risks to adaptation, and individual adaptations (or human responses) to altered health states and to therapeutic interventions designed to improve the altered health states (Heitkemper \& Shaver, 1989; Heitkemper et al., 1995). There are four types of human responses: physiological, pathophysiological, experiential, and behavioral. Model components that were considered in this study were: age and gender of the parent as non-modifiable person factors; fatigue, and caregiver burden as the experiential response for the parent; and sleep/activity patterns as the behavioral human response for the parent. 


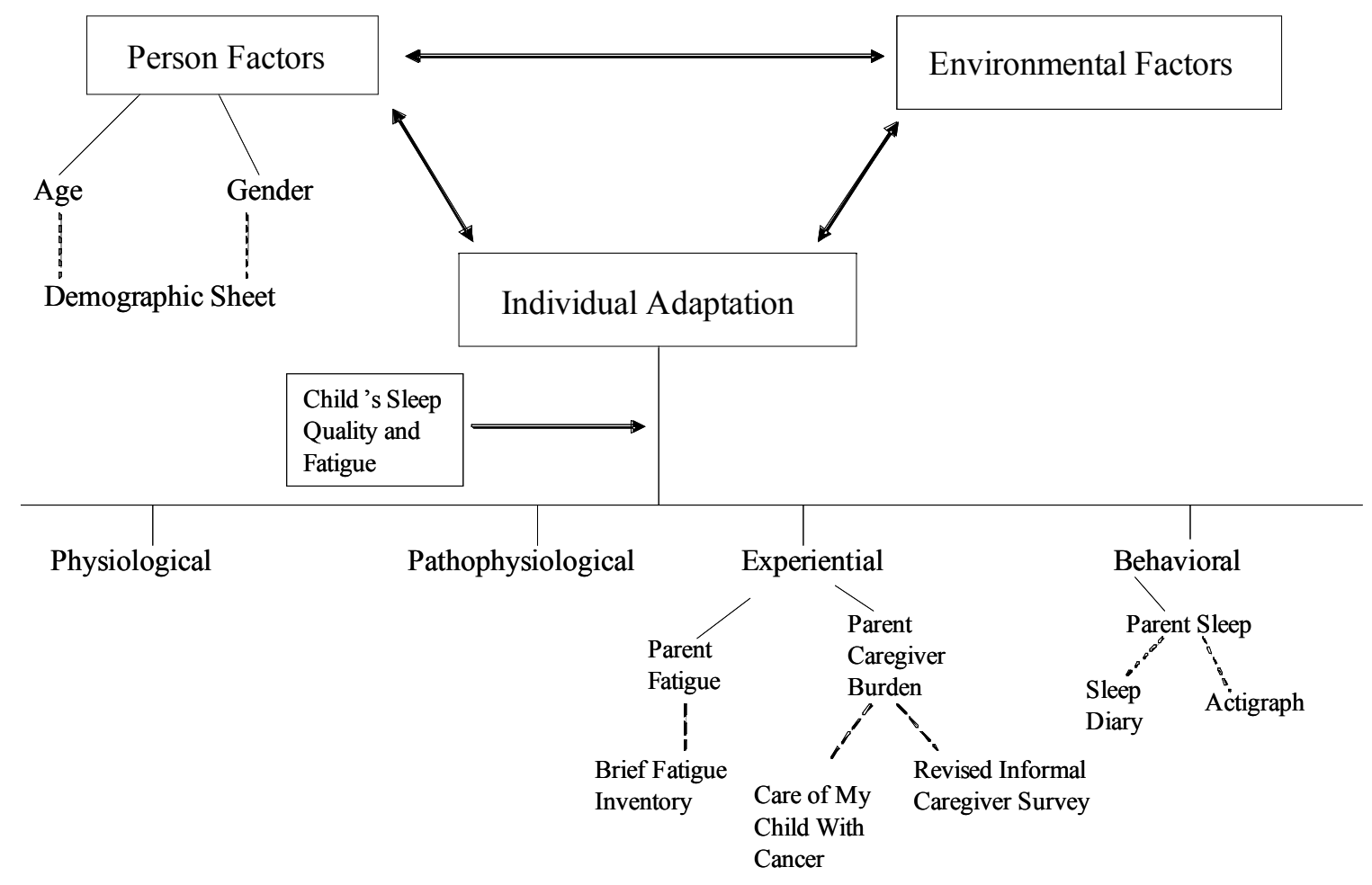

Figure 1-1: Human response model for sleep, fatigue, and caregiver burden in parents of children and adolescents enrolled on the St. Jude Children's Research Hospital protocol, Sleep, Fatigue, and Dexamethasone in Childhood Acute Lymphoblastic Leukemia.

Source. Heitkemper, M., Levy, R. \& Jarrett, M. (1995). Interventions for irritable bowel syndrome: A nursing model. Gastrointestinal Nursing, 18, 224-230. Permission to use model granted by $\mathrm{M}$. Heikemper. 
Theoretically, a change in one human response could alter one or more of the other human responses. As indicated in the primary aim of this study, the potential effect of dexamethasone on the child's fatigue and sleep as studied in SLEEP may indirectly affect the parent's sleep, fatigue, and caregiver burden.

\section{Definitions of Major Concepts}

The variables and concepts in this study are defined using current literature and inferences from the framework that guided this study. For the purpose of this study, the following definitions were used.

\section{$\underline{\text { Caregiver Burden }}$}

Strains experienced by caregivers, including work adjustments, sleep disruptions, financial strain, physical strain, feelings of being overwhelmed, and responding to change in the child's behavior. For the purpose of this study, caregiver burden referred to the physical, psychological, social, and/or financial reactions that can be experienced in care giving as measured by the Care of My Child with Cancer Instrument (CMCC) and the Revised Informal Caregiver Survey (RICS) (Martinson et al., 1999). See Appendix A and $B$.

\section{Objective Burden}

Objective burden refers to the events and activities associated with the caregiving experience. This type of burden can be perceived as negative (Chou, 2000). Objective burden includes the amount of time, effort, and perceived difficulty required to carry out the caregiving task (Oberst et al., 1989). Permission was granted to use the Care of My Child with Cancer Instrument (CMCC) to measure objective burden (Keegan-Wells et al., 2002) (Appendix A).

\section{Subjective Burden}

Subjective burden is defined as the feelings, emotions, and attitudes related to the caregiving experience (Chou, 2000). Subjective burden was measured using the Revised Informal Caregiver Survey (ICS) for parents (Tidwell, Unpublished Data). (Appendix B). 


\section{$\underline{\text { Sleep Efficiency }}$}

Sleep efficiency is a mathematical term defined as amount of actual sleep in minutes divided by the time spent in bed or trying to go to sleep (Landis, 2002). Sleep efficiency was measured using a wrist actigraph.

\section{Sleep Quality}

Sleep quality includes several subjective and objective components of sleep. Subjective components include depth of sleep, how well rested one feels upon awakening, and general satisfaction with sleep. Many questionnaires have been developed to capture and quantify sleep quality (Pilcher et al., 1997). In this study subjective sleep quality was assessed in question numbers 11,14 , and 15 of the sleep diary (Appendix C). Objective sleep quality includes nocturnal awakenings, sleep duration, sleep efficiency, total sleep time, wake after sleep onset, and actual sleep time (Sadeh et. al., 1995). In this study objective, sleep quality is measured by actigraphy and sleep diary reports.

\section{$\underline{\text { Fatigue }}$}

For the purpose of this study, fatigue is defined from the caregiver's perspective. Fatigue is defined to as "a subjective sense of weariness or tiredness resulting from exertion or stress or as a condition of impaired efficiency, resulting from prolonged mental and/or physical activity or from an attitude of boredom or disgust from monotonous work" (Mindell \& Durand, 1993). Fatigue experienced by the caregiver can be physical and psychological. Permission was given to use the Brief Fatigue Inventory (BFI), a self-report instrument, to measure fatigue in this study (Mendoza et al., 1999; Anderson et al., 2003). (Appendix D).

\section{General Definitions}

\section{$\underline{\text { Acute Lymphoblastic Leukemia }}$}

Acute lymphoblastic leukemia (ALL) is the most common childhood cancer. Children with ALL have an increased growth in immature white blood cells or lymphocytes. These cells crowd out normal blood cells in the bone marrow and can spread to other organs including liver, spleen, lymph nodes, central nervous system, kidneys, and gonads. Approximately 85 percent of the diagnosed childhood acute leukemia in the United States is ALL (Pui, 2006; Steen \& Miro, 2000). 


\section{Parental Caregivers}

Parental caregivers are defined as the individuals assuming the mothering and fathering roles toward the child within the family. Both female and male parents/guardians were eligible to participate in this study.

\section{Primary Caregiver Burden}

Primary caregiving includes responsibilities for illness care, physical care, and meeting the social and developmental needs of the ill child (Stewart et al., 1994).

\section{$\underline{\text { Secondary Caregiving Burden }}$}

Secondary caregiving burdens include meeting the psychological needs of other family members, and maintaining activities outside the home (Stewart et al., 1994).

\section{Informal Caregiver}

Informal caregiver is a term that is usually used to describe persons who assume care responsibilities for a family member who has a chronic condition (McGarry \& Arthur, 2001). Informal caregivers can live with the care recipient and much of their time is spent providing care in varying degrees to loved ones. Parents are one kind of informal caregiver

\section{Sleep}

Sleep is a physiological activity that is fundamental to normal daily functioning and well-being. Sleep deprivation may lead to serious problems in daily life, such as low energy levels, feeling tired, sleepy, and irritable and difficulty concentrating (Landis, 2002).

\section{Actual Sleep Time}

Actual sleep time is total number of minutes of night time sleep as measured by actigraphy (Sadeh et al., 1995).

\section{$\underline{\text { Sleep Duration }}$}

Sleep duration is the total number of minutes in bed reported by sleep diary and total nighttime sleep measured by actigraphy (Sadeh et al., 1995). Participants in this 
study were asked to document their sleep duration in two items in the sleep diary: the time they went to bed and the time they actually got out of bed. Richards (1996) describes this time period as time in bed or time from lying down in bed to arising.

\section{Total Sleep Time}

Total sleep time is a calculation of sleep period minus time spent awake as measured by actigraph. This is described as total minutes of sleep and time in bed in a 24hour period. This time includes naps (Sadeh et al., 1995).

\section{Sleep Latency}

Sleep latency is defined as the time when one actually decides to go to sleep and gets into bed until sleep onset (Richards, 1996; Landis, 2002). This is recorded in the sleep diary as the time that it may take one to actually fall asleep.

\section{Wake after Sleep Onset}

Wake after Sleep Onset (WASO) is actual arousal after falling to sleep as measured by actigraphy (Richards, 1996). This is documented as interruptions in sleep. The participants in this study were asked to document how many times their sleep was disturbed and for how long they were awake.

\section{Dexamethasone}

Dexamethasone is a systemic corticosteroid that is long-acting. It has a potency that is about 25 times greater than the short-acting corticosteroids (Zoorob, 1998). Dexamethasone is used as standard therapy for children with ALL. Use of this steroid has significantly decreased the incidence of relapse disease for all risk group children with ALL (Mitchell et al., 2005).

\section{Background on Sleep, Fatigue, and Dexamethasone in Childhood ALL Study (SLEEP)}

Corticosteroids are vital in treating childhood ALL. Dexamethasone has become the corticosteriod of choice for this disorder due to its ability to provide better central nervous system penetration and its cytotoxic effect on leukemic lymphoblasts (Igarashi et al., 2005; Kaspers et al., 1997). Dexamethasone, although significant in the treatment of ALL, also has some associated side effects such as fatigue and changes in sleep, emotions, and behavior (Drigan et al., 1992; Harris et al., 1986; Watanabe et al., 1994). 
Such changes may be linked to individual variability in the systemic exposure to dexamethasone. This link may have implications for the dosing schedules of dexamethasone in the treatment of childhood ALL. The overall purpose of the SLEEP study was to determine the relationship between systemic exposure to dexamethasone and child's sleep and fatigue by comparing child sleep and fatigue indicators in two consecutive 5-day periods during continuation therapy for childhood ALL off and on dexamethasone (Hinds et al., 2007).

\section{Significance of the Study}

Cancer affects 1 in 600 children before the age of 15 and more than seventy percent are cured (Craft, 2000). Despite the cure rates, the burden of caring for a child with cancer can have life-long effects on the parent caregivers. Parents are responsible for providing health care at home as well as managing every day responsibilities such as household chores, bills, and other children.

The care of the child with cancer is essentially in the hands of the parent. Care includes responsibilities of illness, physical care, and social and developmental needs of the child. Parents are overwhelmed with the diagnosis and wealth of information needed to properly care for their child. Sullivan-Bolyai et al. (2003) suggest that while much education is done preparing parent caregivers for the physical care of the child, little attention is given to educating and supporting these parents to manage the stress and the variety of tasks awaiting them once they have been discharged home.

Care of the pediatric patient includes care of the family as well. Parental health can be influenced by the health of the child. Parents often report that their child, diagnosed with ALL and receiving steroid therapy, experiences altered quality of sleep such as inability to sleep, delays in falling asleep, or sleeping only sporadically. As a consequence, parents' sleep can be disrupted and fatigue can result. Disrupted sleep in parents of ill children may lead to increased fatigue and errors in caregiving. Proper attention to the needs of the caregiver is essential to the care and health outcome of the pediatric patient.

\section{Assumptions}

The assumptions for this study included:

1. Parents will honestly report time and effort taking care of their ill child.

2. Parents can self-report their subjective burden especially those items related to emotional or personal aspects of caregiving.

3. Manipulation of the care environment can adversely affect individual adaptation. Studies by Phipps et al. (2004) and Lahteenmaki et al. (2004) suggest that there are both physical and emotional adaptations that parents have to make to assume the unfamiliar care responsibilities for their child 
with cancer. This study assumes that there is a reciprocal relationship between the ill child's health and the parents' caregiving ability, level of fatigue, and sleep quality.

4. The wearing of actigraphs and completing self-report measures would not be too burdensome.

5. Parents' past experience can affect other forms of adaptation to the child's illness.

6. Caregiver burden is described as a dichotomous concept, composed of subjective and objective burden (Chou, 2000). Both dimensions of burden are measured and considered outcomes of the caregiving process in this study. Parental caregivers caring for their chronically ill child have been reported to experience both subjective and objective burden (Phipps et al., 2004; Boman, 2004).

7. Caregiver burden changes as the demands of care change and/or the caregiver adapts to the care demands.

\section{Limitations}

There are several limitations related to the design and methodology of this study:

1. The Human Response Model (Figure 1-1) used in this study was previously untested in clinical investigations. Therefore there is limited documentation to support the theorized relationships. This model has not been tested in this study population.

2. Because the study was conducted after the first year of treatment, it is possible that a change may not be detected in caregiver burden, sleep, or fatigue as parents may have adapted to the care-related situation by this point in treatment.

3. The term "burden" can carry negative connotations and may decrease the likelihood of parents' honesty.

4. The findings in this study do not represent all eligible participants. The most burdened caregivers may have been those who did not participate.

5. Eligibility of participants was dependent on participation of their ill child in the child's protocol (SLEEP).

6. Hardships such as care of other dependent children in addition to the ill child could have influenced the parents' responses to the caregiver burden measures but they were not measured in this study.

7. The participants were informed in the consent process for this study and in the consent process for their child's treatment protocol that their child was receiving dexamethasone and that sleep and fatigue was being studied as related to dexamethasone treatment. This disclosure may have heightened awareness of the study purpose and could have affected parental attention to sleep, fatigue, and burden and their responses to the study questionnaires. 
8. This convenience sample was taken from a unique population.

Convenience sampling may lead to some selection bias. (Burns \& Grove, 2001).

\section{Summary}

Despite the noted limitations of this study, there are unique qualities about this study as well. Previous efforts to measure parental caregiver burden primarily report only the maternal experience and rely upon self-report measures. Little is documented about gender differences in parental caregiver burden in parents whose child has cancer although gender differences in anxiety in adult caregivers of ill spouses or parents have been previously reported (Lutzky \& Knight, 1994). The phase in treatment of the ill child was not routinely reported in published studies on parental caregiving. In addition, change over time in parental caregiver burden corresponding to the ill child's phases of treatment was not reflected in study designs. Both mothers and fathers were eligible to participate in this descriptive study. Trends related to potential gender differences in parental caregiver burden and other study variables were examined in this study in an exploratory manner. Parental adaptation responses were measured during a specific point in their child's treatment and at the very same time when the child's adaptation was being measured.

An additional strength of this study is that both subjective and objective measures were used to document parental outcome indicators of adaptation. Most measures of caregiver burden are comprised solely of negative items and do not include items related to potentially beneficial aspects of this role (Schofield et al., 1997; Caserta et al., 1996). More recently developed and tested measures seek both the negative and positive reactions to caregiving but these are primarily focused on adult caregivers providing care for ill adults (Schofield et al., 1997; Nijboer et al., 2001; Nijboer et al., 1999). Most of the published studies that examined subjective parental caregiver burden were qualitative studies. This current study was a longitudinal descriptive study that used quantitative methods to measure subjective and objective burden specific to parents with children diagnosed with cancer. Both the negative and positive aspects of parental caregiver burden are documented in this study.

Researchers using theoretical models to study caregiver burden often used stress and coping-stress process models. This study utilized the Human Response Model (Figure 1-1). Use of this model in a caregiver burden study is unique, and may help explain the influence of the child's health and treatment regimen on parental burden and other factors influencing parental health. 


\section{CHAPTER 2: REVIEW OF LITERATURE}

\section{Introduction}

Recently published literature has focused on the impact of caring for a chronically ill child at home. Researchers report that providing care for children with chronic illnesses such as diabetes, cancer, and sickle cell disease can cause psychological stress (Boman et al., 2004). Boman (2004) stated that parental stress related to childhood illness depends on the type of illness and time passed since diagnosis. Even though parental caregivers of children with chronic diseases share similar experiences, this current study focuses on parents of children diagnosed with ALL during steroid therapy. Treatment for ALL is intense. Children diagnosed with ALL taking high dose steroids may experience behavior changes, sleep changes, fatigue, myopathy, avascular necrosis, weight gain, osteopenia, and acute liver enlargement (Mitchell, 2005). These symptoms are likely to indirectly affect caregivers and are likely to contribute to the amount of strain experienced (Gedaly-Duff et al., 2006; Svavarsdottir, 2004a). Despite the severity of symptoms experienced by children undergoing treatment for ALL and their potential effects on caregivers, there are limited published studies examining the effect of these symptoms on the well-being and caregiving abilities of parental caregivers. Information obtained from this study pertaining to parental caregiver burden, sleep quality, and fatigue reported by family caregivers of pediatric patients with ALL may assist healthcare providers to develop appropriate interventions that are designed to anticipate needs of caregivers and prevent errors in caregiving. An overview of the literature related to general parental caregiving, caregiver burden, caregiver gender, parental sleep, parental fatigue and effects of steroid therapy on children in treatment for cancer will be discussed in this chapter. There are very few published studies utilizing the Human Response Model, but multiple studies about the components of the model have been published. Information about the individual (parental) responses to a child's illness (fatigue, sleep quality, and caregiver burden) is included in this chapter.

\section{Caregiver Burden}

Approximately 15 million to 25 million adults in the United States currently provide informal care (unpaid care) to relatives and friends (Navaie-Waliser et al., 2002). Estimates project the economic value of informal caregivers' services at $\$ 196$ billion. This figure exceeds national spending for home health care and nursing homes (NavaieWaliser et al., 2002). Caring for ill family members can be difficult. Recent research on informal caregivers has focused on the intense emotional and physical burdens on some caregivers (Donelan et al., 2002).

Most of the published work on caregivers reflects a concern that health care professionals need to focus on the health needs and well-being of those charged with the task of caring for ill family members. Caregivers with unmet needs or high levels of caregiver burden may experience difficulty in their carrying out their caregiving 
responsibilities. If the caregiver burden becomes excessive, the health outcomes of ill family members may suffer (Deeken et al., 2003). Burdens experienced by caregivers can include work adjustments, sleep disruptions, financial strain, physical strain, feelings of being overwhelmed, and responding to change in the patient's behavior. For the purpose of this study, caregiver burden will refer to the physical, psychological, social, and/or financial reactions that can be experienced in caregiving.

There are several approaches used to examine the concept of caregiving and caregiver's burden. A 20- year (1980-2000) review of caregiving research by DilworthAnderson et al. (2002) summarizes the theoretical approaches used to study caregiving. In their review of 32 studies, they found that 14 studies used identifiable theoretical approaches. Thirteen of the 14 articles that used theoretical approaches used conceptual models and one used a conceptual framework and 18 used empirical generalizations (inferences not guided by theory or conceptual models). Some of the theoretical approaches used included: Anderson and Newman's behavioral model, the structural model of caregiving, the illness help-seeking model, the hierarchical model of support, the task specificity model, and the stress and coping -stress process models.

Another recent study described caregiver burden in a cultural context using Leininger's Sunrise Model, (a conceptual framework that incorporated culture and care as an entity in nursing ) (Leininger, 1991) to provide a guide for uncovering the views of caregivers of children with cancer in Taiwan (Liang, 2002). One of the more frequently used models in the caregiving literature is Pearlin's Stress Process Model. This framework describes caregiving as a process in which the caregiver's physical and psychological stamina is depleted with exposure to long-term stress (Pearlin et al., 1990). This model, originally developed for research in caregivers of persons with Alzheimer's disease, has since been applied to other populations including caregivers of persons with cancer (Schumacher et al., 1993 \& Nijboer et al., 1998).

Raina et al. (2004) conducted a review of literature comparing studies that investigated health and well-being of caregivers of children with disabilities with that of the health and well-being of caregivers of the elderly. As a result of this review, a multidimensional comprehensive conceptual model was proposed that depicts the caregiving process and caregiver burden among the pediatric population. The model includes relationships among the family background, child characteristics, caregiver stress, intrapsychic factors (i.e., role performance, self perception, and self-coping factors and caregiver health outcomes (physical and psychological). The researchers concluded that while caregiving is a normal part of being a parent, providing high level care to a child with a chronic illness can become burdensome and may indeed negatively impact the health of the parent caregiver.

\section{Parental Caregivers of Chronically IIl Children}

In the pediatric literature, parental caregiving has been conceptualized in terms of primary and secondary burdens (Stewart et al., 1994). Primary caregiving includes 
responsibilities for illness care, physical care, and meeting the social and developmental needs of the ill child. Secondary caregiving burdens include meeting the psychosocial needs of other family members, and maintaining roles and activities outside the home. Miles and colleagues (1993) offer a somewhat different conceptualization of parental caregiver burden that includes three additional roles: advocating (ensuring the child's special needs were met), protecting (being vigilant to prevent treatment complications), and nurturing (supporting the child's coping efforts).

There are several studies that address the concerns surrounding parenting children with chronic illnesses. A literature review by Margaret Miles (2003) identified seven current programs of research that provide insight into families with children with chronic health problems. Two of the programs focused on children with cancer, three programs focused on a variety of childhood chronic conditions, one focused primarily on parents of children with Down Syndrome and disabilities, and the last program of study focused on parents of infants with a variety of chronic health problems. The studies concentrated on coping, family stress, age of the child, parental gender differences on parental perceptions of the child's illness, parenting, caregiver burden, family support, healthcare provider support and family responses to illness. This review revealed several common observations about families with chronically ill children including:

1. A critical period for these families is the time of diagnosis (Miles et al., 1998).

2. Support from the health care team is a very important factor in parental coping (Garwich et al., 1995; Knafl et al., 1993).

3. There were both positive and negative outcomes in caring for an ill child (Knafl et al., 1993; Miles et al., 1999 \& Van Riper, 2000).

4. Parents for the most part reported that they could provide the care needed for their ill child. Even though caring for an ill child became part of "everyday" life, there were still some caregiving experiences that were burdensome (Knafl \& Zoeller, 2000).

5. Illness was a major factor that affected family life and became a source of burden for family life (Knafl \& Deatrick, 1986).

6. A strategy used by families to cope and manage with their child's illness is normalization (Miles, 2003).

7. The father's perspectives on caring for an ill child are being addressed in many recent studies (Miles, 2003).

This review provided insight into designs and needs for future research about families with chronically ill children and the need to explore child and family outcomes. It also reiterated the need for clinical attention to the needs of these families.

Several other studies have investigated the psychological adjustment, stress and family functioning in parents of children with chronic health conditions. According to Pradeep et al. (2004), chronic illness in children affects the psychological health of the parents and can negatively impact the parents' finances as well. Mothers of chronically ill children are often described as lacking self-confidence, depressed, neurotic and 
introverted. Fathers of chronically ill children are described as having personality changes that can be long term (Cummings, 1976). In a study of parent caregivers of children with HIV, high rates of psychological distress were observed. However, the high level of psychological distress was linked to levels of stress of daily living and emotion-focused coping styles (Bachanas et al., 2001).

Sepa et al. (2004) studied the correlates of parenting stress in Swedish families of children with insulin dependent diabetes and identified parental dissatisfaction (frustration with parenting role) and poor child sleeping patterns as psychosocial predictors of parenting stress. The results from this study are not congruent with similar studies as they reveal that poor sleep quality predicted more parenting stress than the child's illness.

Caring for a chronically ill child can also impact family functioning. Family functioning refers to the patterns of relationship connecting members of a family system to each other (Patterson \& Garwick, 1994). Rodrigues \& Patterson (2007) examined the impact of the severity of a child's chronic illness on family functioning in 262 twoparent families of infants and pre-adolescents using self-report inventories. Surprising to the researchers, data revealed that the families with chronically ill children functioned as well or better as families with healthy children. Differences were found between mothers and fathers. Mothers of infants and pre-adolescents reported greater negative impact on their role performance while fathers of the pre-adolescents reported greater negative impact on affective expression. Severity of the child's condition was found to be associated with overall family functioning. Perhaps stronger family functioning in these families is due to the development of internal strength, resilience or that overtime the families have adapted to life with a chronically ill child.

Drotar (1997) conducted a review of literature on the relationship of parent and family functioning with the psychological adjustment of children experiencing chronic health conditions. Recommendations for research, from this review included: (1) enhancing generalizability of predictive models of family influences, (2) developing and testing more specific models of family socialization processes, (3) assessing family response to the burden of the child's illness and/or developmental transitions, and (4) utilizing findings from parent and child research to develop and test psychological interventions.

Parents of seriously ill children are recognized as one of the largest relational groups of caregivers (Schofield, 1997). In reviewing literature specific to parents of children with chronic illness, there is evidence that supports giving attention to the various needs of these families. However, there is still a need to investigate illness effects on families of children with specific chronic illnesses during specific developmental ages and as a family unit (including mother, father and ill child). Recently, more studies have focused on parents and families of children with cancer. 


\section{Parental Caregivers of Children with Cancer}

The child with cancer does not exist in isolation but rather in relation to caregivers, mostly the family. At the time of a cancer diagnosis, changes occur in the patterns of family functioning such as daily routines, roles, interaction within family structure and with other support functioning. This is usually a stressful time for families. The diagnosis of childhood cancer may lead to parental health problems such as anxiety, depression, and somatic complaints. These health problems may lead to changes in caregiving practices of family members (Martinson et al., 1999).

Parents are recognized as the primary caregivers for their child with cancer, in large part because of the treatment philosophy to provide as much care on an outpatient or in-home basis as is possible. In a recently completed instrument-testing study, 156 parents of children with cancer rated the time and effort they put into providing daily care for their ill child (Keegan-Wells et al., 2002). They also responded to open-ended questions regarding the impact of the caregiver role on their own health. The majority of their responses identified negative physical and emotional effects on their health. The parents expressed appreciation for periodic relief from direct caregiving and ongoing assistance with household responsibilities (Keegan-Wells et al., 2002). Other descriptive studies have documented the effects of the child's illness on other family members, parents' marital adjustment (Cornman, 1993; Dalquist, 1996; \& Thoma, 1993), and the burden of medical and non-medical expenses that these parents incur secondary to their child's cancer treatment (Birenbaum et al., 1992; Lansky et al., 1979). These preliminary studies indicate that parental caregiver burden is multidimensional and although intensely negative, the caregiver role has some positive aspects for these parents.

Children with ALL are usually treated on an intensive treatment protocol that can last up to 3 years. This treatment time can be very stressful for family members. A randomized crossover study by Stevens et al. (2005) examined quality of life (QOL), safety, caregiver burden and cost of hospital and home chemotherapy for children with leukemia. Eligible children were randomly assigned to either the hospital (standard care) or home (treatment) chemotherapy group. After a 6-month period, the children switched groups for another 6-month duration thus serving as their own control. Data collection was completed in about a year. The Caregiving Burden Scale was used to measure burden in both the hospital and home setting. The results revealed that there was no difference in burden with respect to where chemotherapy was administered. However, age of the child was a significant predictor of QOL and caregiver burden; caregiver burden decreased as the age of the child increased (Stevens et al., 2005).

Having a child with cancer is described as a crisis situation for the family and can lead to emotional instability, uncertainty and strain among family members (Svavarsdottir, 2005c), but many parents do adapt to the situation. The parents are occupied with their ill child's medical treatment and adapting to life changes. Family members are either brought closer together due to the illness or driven further apart. In a study exploring the life situation of parents of children with cancer over the first year, the researchers found that although the perceived amount of lost family income was high 
during the first few months, parent attitudes were fairly positive surrounding their family life and spouse relations. Both mothers and fathers in this study rated their own health as being significantly negative (Lahteenmaki et al., 2004). This may be an area for further study and intervention.

In an investigation of patterns of distress in parent whose children were undergoing stem cell transplant, correlations between parent and child distress were documented. Parental distress was unrelated to child age, gender, diagnosis or transplant but was significantly related to parental socioeconomic status (Phipps et al., 2004). There is also a strong relationship between parental mental health and adjustment to their child's illness. Measures of child somatic distress at the time of transplant were not predictive of parental distress, but child mood disturbance at time of transplant admission was predictive of parent distress over time. If the child's mood disturbance was low then the parents' distress was also low (Phipps et al., 2004). The investigators recommended that interventions designed to reduce distress in parents of children with cancer be implemented.

Children diagnosed with cancer have a chance of being cured of their disease, unlike some other acutely or chronically ill children whose treatment is palliative or short-term only (Wingo et al., 1995). Parents of children with cancer focus their efforts on placing their child in the best position possible for cure and continue those efforts for extended periods of time (Hinds et al., 1996). Because the parent caregiver is the center of support for the needs of the ill child, if the caregiver is unable to provide needed medical care, the child could suffer (Andrews, 2001).

\section{Gender Differences in Parental Caregivers}

Most parental caregiver research has focused primarily on the role of the mother. Little is in the literature about gender differences in parental caregiver burden in parents whose child has cancer although gender differences in anxiety in adult caregivers of ill spouses or parents have been previously reported (Lansky et al., 1979). Both mothers and fathers were eligible to participate in this pilot study. Trends related to gender differences and whether they exist in parental caregiver burden were studied in an exploratory manner.

Involvement of both parents in caregiving is essential. Recent studies have explored the psychological and coping experiences of fathers of chronically ill children. Gender differences have been investigated in variables such as adaptation, perception, social support, self esteem, coping behaviors and marital satisfaction. A study conducted by Shira Katz (2002) investigated the impact of a child's chronic illness on fathers and mothers. Eighty fathers and mothers of children diagnosed with cancer, heart disease, diabetes, asthma, and juvenile arthritis were asked to complete eight questionnaires about psychosocial adjustments to their child's illness. The findings from this study suggest that social support was one of the most important variables affecting coping behaviors of 
the fathers. Perception of illness was one of the more powerful influences on coping behaviors for mothers.

Another study by Chao-Hsing Yeh (2002) investigated gender differences of parental distress in Taiwanese mothers and fathers (164 couples) whose children were diagnosed with cancer. Mothers and fathers in this study had similar patterns of stress and distress but the mothers averaged higher levels of distress than fathers. Parents (both mothers and fathers) of children who had been recently diagnosed (during the past 2 months) reported greater depression, anxiety and marital dissatisfaction than parents whose children were further along in treatment for cancer. However, both fathers and mothers of children in the off-treatment group reported higher levels of parenting stress. Mothers with children in the relapse group had the highest level of stress in the parentchildren dysfunction index. Fathers in the newly diagnosed group also complained of somatic symptoms associated with depression. Marital satisfaction was perceived differently by mothers and fathers in this study. Fathers generally were consumed with supporting the family financially while mothers were busy with caregiving for the sick child.

Research studies have shown that mothers and fathers of children with cancer experience their child's illness differently and cope with the illness in different ways. These parents also encounter stressors that vary over time. Findings from longitudinal research with families of children with cancer indicate that mothers have less anxiety 20 months into treatment than at 2 months after diagnosis. Fathers' anxiety, on the other hand, did not change over time (Dalquist et al., 1996).

In another longitudinal study of parents caring for children with cancer, Svavarsdotti (2005b) evaluated caregiving demands, perceptions, and well-being among fathers and mothers of children with cancer in Iceland. Data were collected at baseline, 12 months and 18 months between 1999 and 2001. Mothers reported the most difficult caregiving task was providing emotional support for the child. Mothers reported that the most time consuming tasks were providing emotional support for the ill child and for the siblings and coordinating services and resources for the family. Fathers in this study reported the most time consuming caregiving task was providing emotional support for the ill child, siblings and spouse. The most difficult task for fathers was providing emotional support to the ill child and to his spouse, and managing work outside the home.

\section{$\underline{\text { Mothers }}$}

The actual burden of caregiving in families with chronically ill children is generally assumed by the mother (Martinson et al., 1999). Mothers tend to be more active in the care of the sick child. Most times the mother makes treatment decisions, administers the medication and stays with the child during hospital admissions. Eiser et al. (2005) found significant correlations in quality of life (QOL) in mothers and their children with newly diagnosed cancer. Both the children and their mothers reported significantly lower QOL than population norms. A positive correlation between 
caregiver burden and parenting control was identified in a survey of maternal distress and child rearing strategies during treatment for childhood cancer. It is proposed that increased parental control may be a valuable adaptive response to increased caregiver burden. While maternal distress decreased post-initial diagnosis, maternal caregiver burden was found to remain fairly constant after 24 weeks of treatment (Steele et al., 2003).

It is difficult to separate the meaning and task of caring for an ill child from the innate responsibilities of nurturing and caring for a child that is not diagnosed with chronic illness. Separating these tasks and feelings can be difficult to describe, quantify or psychologically comprehend. Mothers are more commonly seen as the caregiver in the family. Therefore it may seem socially unacceptable to discuss maternal concerns about the physical and emotional strains related to caring for a chronically ill child. It is easier to comprehend care for an ill child as the "normal" part of being a mother. In reality, this becomes a different normality for mothers of a child with ALL.

Earle et al., (2007), explored what "building a new normality" meant to mothers of children diagnosed with ALL. Thirty mothers were interviewed at 3 time points (3-4 months post- diagnosis, 15 and 27 months respectively). These mothers reported understanding that it was important to achieve a normal life but the reality of achieving this was difficult. Common themes that emerged from the interviews included recommendations from professionals to lead as normal a life as possible, the effect of the child's illness on daily life, barriers to a normal childhood for the ill child, parenting concerns and strategies to achieve normal life. At the point of 3-4 months following diagnosis, the mothers expressed concerns about the likelihood of their child's illness changing the behavior and personality of their child, disrupting normal work life, and being able to maintain discipline (Earle et al., 2007).

At the second data point in the study (15 months after diagnosis), many of the families were displeased that they had not achieved normalcy after completion of intense therapy. While some mothers began to speak of clinic visits as normal, other mothers felt as though striving for normalcy was an attempt to distract themselves. The concerns about risk of infection and injury continued to be major worries (Earle et al., 2007). Interestingly, during time 3 (3 to 27 months after diagnosis) of this same study, the mothers' focus was directed on normal parenting styles, avoiding treating the ill child differently, more normal life style, and coping by forgetting about tasks surrounding the illness until it was time to address them. Some mothers stated that they did not know how great normalcy was until they did not have it and some Mothers described "the highs and lows" associated with the illness as normal. Parents also felt that their child had resumed a normal personality and behavior by the third time period (Earle et al., 2007). Mothers may benefit from interventions that are specific to the concerns of the female caregiver and to the stages of treatment. Normalization may mean something different to fathers of children diagnosed with chronic illness. 


\section{$\underline{\text { Fathers }}$}

Recent parental caregiver literature has expanded its focus to include the fathers' perspectives. The father's role has commonly been characterized as the protector, communicator, breadwinner and teacher (Hovey, 2005). Fathers, in a study of the impact of childhood cancer and other chronic diseases on parents, felt that the child's illness had a negative effect on their functioning in the family (Katz, 2002). In a descriptive study of fathers of chronically ill children, fathers identified concerns that included their child's health, doing family activities, making the family happy and comfortable, talking with the family, extra demands on time, sexual relationship with spouse and enough fun and relaxation. Of the 48 fathers who completed this study, $13 \%$ of the fathers turned to alcohol, drugs, or smoking as a coping strategy (Hovey, 2005).

Fathers also have to find normalization in an otherwise stressful experience. Peck and Lillibridge (2005) conducted a qualitative study to explore the experiences of fathers living with their chronically ill children in Australia. The four fathers described accepting their child's illness, changing expectations that they had for the child, focusing energies on a daily basis, using knowledge -seeking behaviors and engaging in activities outside the home as ways to normalize the stressful experience of living with a chronically ill child. These fathers also saw employment as a getaway from the disarray at home. Much like the mothers in a previously discussed study, the reality of having a child with a chronic illness made normalization difficult for fathers.

Parenting stress and psychosocial adjustment have also been examined in fathers of children with HIV/AIDS. Fathers with children diagnosed with HIV/AIDS scored higher in psychological distress and demonstrated higher levels of parenting stress compared to fathers of healthy children. Both findings were congruent with findings from other studies of fathers with chronically ill children (Wiener et al., 2001). These fathers indicated that there was a need for support services and that they would utilize these services if they were available. This further emphasizes the need for gender specific parental interventions in families with a child diagnosed with a chronic illness.

A diagnosis of cancer can be associated with terminal illness. However, most fathers do not interpret a cancer diagnosis in this way. As long as treatment options are available, fathers often times do not allow themselves to think about death (Davies et al., 2004). Despite these findings, fathers are profoundly affected emotionally by their child's diagnosis of cancer. Fathers report strain secondary to managing the house responsibilities and work and separation from the ill child during hospital admissions (Mcrath, 2001).

\section{Sleep}

Sleep is a physiological activity that is fundamental to normal daily functioning and well-being. Landis (2002) defines sleep as a rhythmic, temporary, perceptual disengagement from and unresponsiveness to environmental surroundings that is 
regulated by a circadian process. Despite extensive studies, the function and purpose of sleep remains ambiguous. It is thought that sleep is needed for recovery, regeneration and realignment of cells, tissues, and organs (Eidelman, 2002).

Sleep is divided into two states: non rapid eye movement (NREM) and rapid eye movement (REM) sleep. Most of night sleep is spent in NREM sleep. Sleep is divided in a cyclic pattern across the night. Restricting sleep to less than five to six hours a night can cause a disruption in the cyclic pattern of REM and NREM (Landis, 2002). The average adult sleep requirement is about 7.5 to 8 hours of sleep regardless of environmental or cultural differences. However, Americans are sleeping less due to schedules and life demands (Chokroverty, 1999).

Sleep deprivation may lead to serious problems in daily life, affecting performance and mood (Marquis, 1996). Sleep deprivation could also lead to fatigue, poor coping skills, increased daytime sleepiness and mistakes in caregiving duties. Experimental acute restriction of sleep has been found to reduce daytime alertness and to disturb mood (Marquis, 1996). Some documented health related changes that affect sleep include depression, anxiety, pain, and acute and chronic illnesses. Factors such as financial concerns, illness of significant others, and stress may increase sleep disturbances such as sleep latency, awakenings, and distressful dreams (Richards, 1996).

Pilcher et al. (1997) looked at both sleep quality and sleep quantity in relation to health, well-being and sleepiness. Thirty-nine college students participated in the study. On average, participants slept about 7 hours a night. The Stanford Sleepiness Scale (SSS) and the Pittsburg Sleep Quality Index (PSQI) were used to measure sleep quality, and the Epworth Sleepiness Scale (ESS) was used to measure sleepiness. Poor sleep quality was significantly correlated with increased physical health complaints and elevated tension, depression, fatigue and confusion. Health and well-being were more closely related to sleep quality than sleep quantity. The results also showed that as time in bed and time asleep decreased, feelings of fatigue, and confusion increased.

Certain limitations do exist in the available research about adult sleep, including that the majority of studies completed to date have been done with male participants. The few studies that included women tended to be about sleep in women who are 50 years of age and older. A study by Lauderdale et al. (2006) examined the characteristics among 669 early-middle aged adults (men and women) using actigraphy and found that sleep duration and sleep quality were closely associated with race, sex, socioeconomic status and health status. Participants were aged 38-50 years old. For the entire sample the mean sleep duration was 6.1 hours (SD, 1.2) and mean sleep efficiency was 80.9 percent (SD 11.3). Average sleep duration for White women was 6.7 hours, 6.1 hours for White men, 5.9 hours for Black women and 5.1 for Black men. The researchers also found that the actigraphy-measured duration was shorter than self-reported sleep duration.

In another study, the sleep of 273 healthy volunteers of men and women aged 4060 years was measured using actigraphy. Results of this study revealed mean sleep duration was 6.2 hours, mean time in bed was 7.5 hours and mean sleep efficiency was 
83 percent. Significant race and gender differences were found in this study also. Women had more time in bed than men and longer sleep duration. Whites showed shorter sleep latency and greater sleep duration and efficiency than Blacks (Jean-Louis et al., 2000).

A study by Jean-Louis et al. (1999) also found that gender had a significant effect on sleep. Irrespective of age, women had statistically higher sleep efficiency $(\mathrm{F}=7.14, p$ $=<0.01)$ and statistically higher latency to sleep onset $(\mathrm{F}=3.74, p=<0.05)$ than men. In this same study, analysis of men's responses to the Stanford Sleepiness Scale indicated men were significantly less sleepy than women. Female sleep patterns can be affected by reproductive hormones and menstrual status (Shaver, 2002). Although there are reported gender differences for some sleep characteristics in adults, little is known about the sleep of adults who are parents of chronically ill children.

\section{Sleep Measurement}

In preparation for this study, a review of studies related to adult sleep was completed to identify the methods most commonly used to measure sleep in adults. Ten different instruments to measure sleep were identified. Actigraphs were the most commonly used measure of sleep followed by sleep diaries (Tidwell, 2003). Sleep diaries are widely used in clinical and research settings and have been documented to be a reliable and useful tool to document sleep/wake patterns. Sleep logs or diaries are often used in research in conjunction with other methods of measuring sleep, and in primary care practice as the first step to establishing the extent and type of sleep quality. Diaries provide a way to monitor sleep habits over long periods of time and are a necessary component in the behavioral evaluation of sleep in field research studies (Tidwell, $\mathrm{J}$ unpublished data; Rodgers et al., 1993; Usui et al., 1999; Usui et al., 1998).

Most of the reviewed studies used more than one instrument to measure sleep. Various combinations of instruments were used to measure sleep. However, the most common combination was the use of the sleep diary and the actigraph (Lockley et al., 1999). The literature reviewed was about adults but few studies addressed parental sleep. The results of this literature review suggest that clinical researchers not rely on any single measure to completely capture sleep quality (Lockley et al., 1999). Subjective and objective aspects of sleep are considered essential by clinical investigators, and no single measure is currently used to capture both of these aspects. It is highly suggested that sleep diaries be used simultaneously with actigraphy whenever possible. (Rodgers et al., 1993; Usui et al., 1999; Usui et al., 1998, Lockley et al., 1999).

The findings of this literature review indicate that a standard approach to measuring parental sleep, based on frequency of their use, includes both a sleep diary and actigraph. Therefore, this study used actigraph and sleep diary to measure parental sleep quality. Sleep diary data were used to corroborate actigraph data and provide points of reference for analysis. Items from the sleep diary that were corroborated with actigraph 
data include: bedtime, wake time, number of awakenings, number of naps, rise time estimates, time it took to fall asleep (sleep latency) and estimation of sleep duration.

\section{Child Sleep}

Sleep disorders have become a more common problem in pediatrics. Approximately $25 \%$ to $40 \%$ of children and adolescents have some type of sleep disorder. There are several different types of sleep disorders that affect youth. These disorders impact the child's daytime functioning, development, growth, learning, behaviors and emotions (Melzer \& Mindell, 2006). A sleep problem or sleep disorder in childhood is described as any sleep pattern that interferes with the amount of sleep required for normal growth and development, emotional, and psychological health and proper immune function (Davies et al., 2004). Common pediatric conditions that are often associated with an underlying sleep disorder are either medical, sleep-related, behavioural or psychological (Goll \& Shapiro, 2006).

Children between the ages of 5 to 13 years require about 10 hours of sleep while those aged 14 to 18 years need about 8 hours of sleep. Excessive sleepiness in a child is defined as sleeping longer than other children of the same age and requiring naps when other children of the same age do not. The most common cause of daytime sleepiness in children is insufficient sleep (Meltzer \& Mindell, 2006; Heussler, 2005).

Sleep problems in children can present as attention and behavior problems, and as depression in adolescents (Heussler, 2005). Parents have described sleep problems in their children with asthma, intellectual disabilities, autism, downs syndrome, and PraderWilli syndrome (Robinson \& Richdale, 2004; Cotton \& Richdale, 2005). Poor sleep habits can have consequences for both children and their parents (Heussler, 2005). Healthcare professionals need to recognize the importance of sleep hygiene for both children and parents.

\section{Parent and Child Sleep}

The National Sleep Foundation (2004a) conducted a survey of more than 600 parents of school-age children (ages 5 to 10 years) and found that $14 \%$ of children were waking at least once a week needing parental help or attention. Almost half of the parents in the survey reported that they attended to their child's waking, resulting in a nightly sleep loss of approximately 30 minutes. Disrupted sleep in children can affect

parental sleep. Studies about sleep problems in children with various disorders and parental effects of a child's sleep problems are prevalent in the literature.

Dahl and El-Sheikh (2007) outline the importance of examining sleep in a family context. The first reason is because sleep problems in one person can often impact family members. Second, sleeping arrangements, stress, attitudes, and emotional environment can influence sleep patterns. Third, sleep in the family environment can influence 
clinical, social and academic aspects of development. Fourth, family context may be an important framework to consider for understanding key aspects of sleep and arousal regulation.

In a study of adverse associations of infant and child sleep problems and parent health, mothers with no past history of depression reported having greater psychological distress associated with infant sleep problems than mothers with a past history of depression (Martin et al., 2007). In this same study, poor general health in mothers and fathers was associated with infant sleep problems; likewise, preschool sleep problems were associated with poor general health in mothers. Management of sleep problems in children could improve general health of parents (Martin et al., 2007). Parental perceptions of their child's sleep, behavior, and emotional status may have a positive or negative influence on the parent's interaction with the child, which is important for family well-being and the child's development (Eckerberg, 2004).

Wiggs and Stores (1998) studied the effect of behavioral treatment for sleep problems in children with severe learning disabilities and challenging daytime behavior. Their study compared the sleep patterns of the mother with that of their child. The behavior modification treatment for the child's sleep problem showed more benefits to the mother's sleep patterns than for their child's sleep patterns. These mothers reported increased sleep, as measured by composite sleep index and activity monitors. These same mothers also reported increased ability to cope with their child's challenging daytime behavior.

Another study by Boergers et al. (2007), studied the association between childhood sleep disorders and mothers' and fathers' sleep duration and daytime sleepiness in 107 families. Parents of children with more than one type of sleep disorder experienced more daytime sleepiness than parents of children with a single sleep disorder. Mothers reported more daytime sleepiness than fathers, although there were no parental differences in sleep duration.

Meltzer and Mindell (2007) also examined the impact of the child's sleep disruptions on the functioning of family members. The researchers examined the relationship between child sleep disturbances and maternal sleep, mood and parenting stress in 47 mothers. Significant differences in maternal mood and parenting stress were found between mothers of children with and without significant sleep disturbances. Mothers of children with significant sleep disruptions reported greater feelings of parenting stress and caregiving overload. Such mothers may be at risk for greater negative feelings toward their child and their own role as a parent. Regression analysis of this study showed that the quality of children's sleep significantly predicted the quality of maternal sleep and that maternal sleep was a significant predictor of maternal mood, stress and fatigue. 


\section{Fatigue}

Fatigue is a multidimensional concept that has been defined and described in various ways within nursing. Fatigue is not the result of daily activities that disappear after a good night sleep but rather a state of decreased capability to mental and/or physical work. Indicators of fatigue include intensity, duration, pattern, domination, specificity and explicability (Tiesinga et al., 1996). Reports have correlated pain, sleep difficulties, psychological distress, poor health, poor social status and depression with fatigue (Berger et al., 2000; Belza et al., 1993; \& Dzurec et al., 2002).

Fatigue is a frequently reported symptom among both cancer patients and family caregivers. Fatigue is due to the disease process and treatment regimen. Fatigue in patients and family caregivers can also be secondary to anxiety related to diagnosis, treatment, and prognosis. Fatigue experienced by the caregiver can be physical and psychological (Jensen \& Given, 1991). Fatigue experienced by family caregivers needs to be explored so that health care providers can help the caregiver alleviate fatigue and effectively provide care to the patient. Fatigue, from the caregiver's perspective, has been defined as "a subjective sense of weariness or tiredness resulting from exertion or stress or as a condition of impaired efficiency, resulting from prolonged mental and/or physical activity or from an attitude of boredom or disgust from monotonous work" (Varricho C., 1985, 13, p. 122). Hinds (1985) studied 83 families who cared for cancer patients at home to determine resources used and to assess families coping with needs of patients. Hinds found that $27 \%$ of families needed guidance with physical care, $13 \%$ needed financial assistance and 53\% identified several other areas where caregiving was fraught with psychological strains that were difficult. Jensen and Given (1991) found that there was a positive relationship between reported severity of fatigue and caregiver's schedule. Fatigue can make the caregiving duties burdensome and may lead to unintentional mistakes in patient's care.

In a recent study of cancer-related fatigue in children and young people, parents, and healthcare providers were asked to complete questionnaires about fatigue in their ill child or patients. Both parents and healthcare providers identified fatigue as a significant problem that has an impact on quality of life. Parents reported that their child's fatigue detrimentally affected their lifestyle as a parent mentally and physically. These parents also indicated that their child's fatigue lead to family disruption because their ill child was unable to perform usual activities without assistance. Parents documented giving "piggy back rides, letting their ill child set the pace, avoiding waking their child at night, carrying food and beverages and setting early bedtimes to help control their child's fatigue. Parents view themselves as having primary responsibility for their child's fatigue, which can be burdensome (Gibson et al., 2005).

Fatigue experienced by mothers caring for ill children can be worsened if the child is hospitalized and is exacerbated by the seriousness of their child's disease. Mental and physical fatigue of mothers caring for chronically ill children can influence the caregiving. Support for fatigued mothers could lead to improved caregiving for their ill child (Iwasaki, 2005). 


\section{Fatigue and Sleep}

Fatigue and sleep are considered to be universal human experiences but fatigue has not received the same careful study as sleep in large part because reliable and valid ways to assess fatigue and effective treatments were lacking (Jensen \& Given, 1991; Glaus, 1993). Sleep quality and well-being of adults have been studied in both small and population-based studies and through self-report and objective measures. In general, sleep duration is not strongly correlated with well-being but sleep efficiency and fatigue are moderately associated with indicators of quality of life and with each other (Floyd et al., 2000; Glaus, 1993; Shapiro, 1998). The relationship between sleep and fatigue in parental caregivers is unknown but disruptions in sleep are theorized to be positively associated with fatigue. Both sleep disruptions and fatigue are believed to be associated with under alertness (Lichstein et al., 1997).

There are few studies that investigate the relationship between sleep and fatigue and even fewer that investigate this relationship in parents. Literature about fatigue and sleep in parents has primarily focused on new parents or mothers of infants and parents of chronically ill children. Ohayon \& Shapiro (2000) describe the relationship between sleep and fatigue complaints as complex and that it is difficult to draw a definite conclusion as to whether sleep disturbances cause fatigue or whether fatigue causes sleep disturbances.

A recent study of Chinese American parents of infants hospitalized in intensive care units described parents' sleep disturbance and fatigue in relation to their stress levels (Lee et al., 2007). Sleep data were collected using three measures, the General Sleep Disturbance Scale, sleep diary, and wrist actigraphy. Fatigue severity was measured using the Numerical Rating Scale-Fatigue and the Parental Stressor Scale: Infant Hospitalization was used to measure stress. Mothers reported greater sleep disturbances and more severe fatigue than did fathers. Mothers also experienced more wakeful time during the night. In both mothers and fathers, reports of higher parental stress and higher morning fatigue were related to subjectively reported sleep disturbances.

A study conducted by Gay et al., (2004) described sleep patterns and fatigue in mothers and fathers of newborns. Seventy-two couples during their last month of pregnancy and their first month postpartum, were asked to wear wrist actigraph and complete questionnaires to estimate sleep and fatigue. Both parents reported more sleep disturbance and fatigue during the first month postpartum. Mothers reported more sleep disturbance than fathers but no difference in fatigue ratings was found. Feeding schedules, parent work status, type of infant feeding, and hormonal fluctuations in these mothers may have contributed to the findings of this study.

Another study by Gedaly-Duff et al. (2006) investigated sleep, pain, and fatigue in children with ALL and their parents. This study demonstrated the trend of symptom clusters within a family unit. Nine children with ALL, 7 mothers and 6 fathers participated in this study. The Child Fatigue Visual Analog Scale was used to measure tiredness, ease of falling asleep, naps, and activity performance in the children. The 
Visual Analog Scale for Fatigue (VAS-F) was used to measure fatigue in the parents. The Parent Three-Day Sleep Questionnaire was used to measure sleep in the adults. Wrist actigraphy was used to assess objective sleep disturbance in the children and their parents. The patients were aged 8 to 18 years, in remission with no relapses and receiving IV chemotherapy at home. Data were collected for 72 hours. The children reported evening fatigue (ranging from 1 to 3 on a scale of 0 to 4 (most tired)) and slept 6 to 10 hours with frequent awakenings. Fathers reported 4.5 to 7.8 hours of sleep per night with frequent awakenings and an average fatigue score of $50 \mathrm{~mm}(0$ to $100 \mathrm{~mm}$; 0 means no fatigue). Mothers reported 6-7.8 hours of sleep per night with frequent awakenings and an average fatigue score of $45 \mathrm{~mm}$ (0 to $100 \mathrm{~mm}$; 0 means no fatigue). These results seem to suggest a link between fatigue and sleep disturbance. The researchers of this study suggest that chemotherapy medications, worry, and changes in family life to accommodate their ill child's treatment schedule may contribute to altered sleep in children with ALL and their parents.

Certain methodological issues regarding sleep and fatigue measurement were identified in the literature. Self-rated sleep quality in adults may be more associated with quality of life than are objective measures, and self-report methods are considered less reliable for number of night awakenings and length of sleep than are more objective measures such as actigraph (Floyd et al., 2000). Actigraphic data have been found to have strong agreement with polysomnographic data from adults and adolescents (JeanLouis et al., 2000), but the actigraph is much less invasive as a measuring device than polysomnography. Time may be an explanatory variable when studying adult sleep and fatigue as gradual sleep restriction does not seem to have the same negative impairment effects on adults as does acute and abrupt sleep restriction (Jean-Louis et al., 2000).

\section{Adverse Effects of Steroids}

Neuropsychiatric adverse effects of steroid therapy include increased motor activity, mood changes, psychosis, vertigo, headache, and insomnia. Short-term, low dose steroid therapy rarely results in any adverse effects listed above. However, as the dosage or duration of therapy increases, so does the risk of these undesirable effects (Zoorob \& Cender, 1998). A study by Moser et al. (1996) on the effects of dexamethasone and sleep reported that dexamethasone in dosages commonly used in clinical situations, lessens sleep efficiency and reduces REM sleep.

Dexamethasone is becoming the drug of choice in clinical trials for standard risk children with ALL despite the concerns that it may have more side effects than previous conventional therapies. A study conducted by Eiser et al. (2006) examined the implications of dexamethasone on health-related quality of life (HRQOL) in children with ALL in the first 3 to 6 months after diagnosis (time 1) and 1 year later (time 2). The children were randomized to either receive dexamethasone or prednisone. The HRQOL questionnaires were completed by the mothers. The mothers from both the prednisone group and the dexamethasone group reported improved HRQOL scores and significantly decreased behavior problems from time 1 to time 2 . The rate of improvement did not 
differ between children randomized to dexamethasone or prednisone. Mothers completed a HRQOL questionnaire for themselves. They also reported an increase in HRQOL at time 2.

There is discrepancy in the literature regarding whether using dexamethasone is more advantageous than prednisolone (Mitchell et al., 2005; Igarashi et al., 2007). Children with ALL on steroid therapy can receive up to $60 \mathrm{mg} / \mathrm{m}^{2}$ of prednisone for 5 days. In a study conducted by Harris and colleagues (1986), 16 children diagnosed with cancer were found to have physical, emotional, and behavioral changes on and off prednisone. Increased irritability, argumentativeness, tiredness, "talks too much," and cries for no reason were the commonly reported symptoms. Nineteen percent of the subjects reported difficulty falling asleep and early morning waking while on prednisone and $38 \%$ reported waking in the night while on prednisone. Major psychiatric disorders are uncommon in pediatric patients receiving corticosteroids, but a case report by Watanabe et al. (1994) found mania and panic associated with dexamethasone in two adolescent girls diagnosed with ALL. These girls did well initially on high doses of prednisone but after dexamethasone (at prednisone equivalent does) was started, major adverse psychiatric effects developed.

A recent review of adverse psychological effects of corticosteroids in children and adolescents substantiate findings from earlier studies in that although rare, adverse psychological effects and psychotic symptoms can occur in children. Some reports suggest that oral dexamethasone treatment may carry a higher risk of adverse psychological effects, however more research is needed to confirm this suggestion. Stuart and colleagues (2005) identified sixteen reported cases of severe adverse psychological effects in children and adolescents undergoing treatment with corticosteroids. One of the cases did involve a child with ALL and three cases involved dexamethasone as part of chemotherapy protocol. Insomnia, tearfulness, irritability, and argumentative behavior and tiredness are documented as common side effects of corticosteroids (Stuart et al., 2005). Although corticosteroids are valuable in treating many conditions in children and adolescents (Stuart et al., 2005; Zoorob \& Cender, 1998), parents and children should be advised of the potential adverse psychological side effects of corticosteroids.

\section{Summary of Review of Literature}

Family caregivers of patients with cancer are often overwhelmed with stress during the caregiving experience. Caregivers loose sleep, which can lead to irritation and anger toward their family member with cancer or other family members. The literature reveals that stress and sleep loss associated with caregiving can lead to depression and other emotional and physical health problems (Carter, 2002; Carter, 2003; Meltzer \& Mindell, 2007; Martin et al., 2007). Recent literature supports the importance of examining symptom clusters such as sleep disturbance, fatigue, and caregiver burden as a family unit (mother and father and child). The literature also supports the use of actigraph and sleep diaries in sleep studies. 
Currently we are not aware of any studies that examine the impact of sleep quality, and fatigue on caregiver burden of parents with children on steroid therapy for ALL. Evaluating the effects of fatigue and sleep quality on parental caregiving duties may help health professionals anticipate needs and assist these families in coping and managing the care of their children and decrease risk of health problems in caregivers. 


\section{CHAPTER 3: METHODS}

\section{Research Design}

This study is a descriptive comparative study with the overall goal being to measure parents' sleep quality, fatigue, and caregiver burden during two consecutive five-day periods and to compare parent sleep quality with that of their ill child. This study is a companion study to the Sleep, Fatigue and Dexamethasone in Childhood ALL study (SLEEP) and the St Jude Children's Research Hospital (SJCRH) Total XV protocol for children with ALL. The SLEEP study is a multi-site study examining sleep, fatigue, and the pharmacokinetics and pharmacogenetics of dexamethasone in children diagnosed with low and standard risk childhood ALL.

Descriptive statistics were used to characterize the study sample. The sample was divided into sub-categories. Descriptive statistics were reported by the following subcategories: primary caregivers, mothers, fathers, and couples. Differences between sleep scores, fatigue scores, and caregiver burden scores for the two consecutive 5-day periods were only calculated and reported for primary caregivers and mothers.

\section{Sample and Setting}

This study included a convenience sample of 22 parent caregivers of children receiving treatment at SJCRH in Memphis, Tennessee. Both parents, if available, were asked to participate. The research participants of this study were parents of children between the ages of 5 and 21 years who met the criteria for enrollment on the SLEEP study.

\section{Inclusion Criteria}

Eligible participants were parents of patients who were diagnosed with ALL and enrolled on the ALL treatment protocol ("Total XV") in continuation therapy. Recruitment was aimed at both parents (mother and father) in order to explore data concerning gender in parent sleep, fatigue, and caregiving.

Criteria for inclusion in this study were:

1. Parent(s) or guardian(s) of a child diagnosed with ALL enrolled on Total $\mathrm{XV}$ and past week 50 in the continuation phase.

2. Parent(s) or guardian(s) whose child is enrolled on SLEEP.

3. At least 18 years of age.

4. English-speaking.

5. Willing to give written informed consent.

6. Resides with the child. 


\section{Exclusion Criteria}

Exclusion criteria for this study were:

1. Parents or guardians diagnosed with sleep apnea.

2. Parents or guardians diagnosed with chronic fatigue syndrome.

3. Inability or unwillingness of legal parent to give written informed consent.

Adults with sleep apnea have been found to have restless sleep, morning fatigue, and increased daytime sleepiness; gender differences in the presence and intensity of these symptoms have also been reported (Leech et al., 1988; Shaver, 2002). Sleep disorders are reported to be present in $80 \%$ of patients diagnosed with chronic fatigue syndrome. In addition, adults with chronic fatigue syndrome tend to nap or spend a part of daytime hours in bed. This may aggravate their sleep disturbances leading to further daytime fatigue (Krupp et al., 1993, Morriss et al., 1993, Ohayon \& Shapiro, 2000).

\section{Instruments}

\section{Demographic Form}

Each participant was asked to complete a Demographic form (Appendix E). The demographic form was a four-page questionnaire developed by the investigator that inquired about the participant's age, marital status, ethnic background, household income, number of dependents, occupation, education, exercise routine, sleep routine, and general health status and child's age and sex. The demographic variables were analyzed using descriptive statistics. Estimated time to complete the demographic form was no more than 10 minutes.

\section{Care of My Child with Cancer Instrument}

Objective caregiver burden in the participants was measured using the Care of My Child with Cancer Instrument (CMCC) (Appendix A) (Keegan-Wells et al., 2002). A literature review was conducted to identify an instrument to measure objective and subjective burden in parents of children with cancer. After an extensive review of the instruments and related articles, only the CMCC was found to measure objective burden in parents of children with cancer (Tidwell, unpublished data).

The CMCC instrument (Keegan-Wells et al., 2002) (Appendix A) was developed by the Nursing Research Committee of the Pediatric Oncology Group (POG). It is a 29item questionnaire that asks objective questions regarding tasks assumed in caring for a child with cancer. There is one open-ended question asking the parents to share any other information they would like to share regarding the care for their child with cancer. The 
highest possible score is 140. Scores for each item in the domains range from 1 to 5 ; with 5 representing a higher demand score. Moderate demand scores range from 2.5 to 3.4. The CMCC has a test-retest correlation coefficient of 0.90 indicating that the instrument is stable across a three to seven day interval. Internal consistency of the instrument was estimated using Cronbach's alpha, which was 0.93. This instrument used content validity; generating items from nursing experts, literature, and parents caring for children with cancer.

In this study internal consistency was estimated for the items within the Physical Care and Emotional Care categories of the CMCC. Internal consistency estimates for CMCC items for Physical Care (PC) are shown in Table 3-1. The items are reliable with standardized Cronbach alpha estimates ranging from 0.96 to 0.97 using a definition of internal consistency being a Cronbach alpha of equal to or greater than 0.70 (Litwin, 1995).

Internal consistency estimates for $\mathrm{CMCC}$ items for Emotional Care (EC) are shown in Table 3-2. The items are reliable with standardized Cronbach alpha estimates ranging from 0.89 to 0.91 using a definition of reliability to be a Cronbach's alpha of greater than or equal to 0.70 (Litwin, 1995).

\section{$\underline{\text { Revised Informal Caregiver Survey for Parents (RICS) }}$}

The RICS for Parents was chosen to measure subjective burden in the parents of this study. The RICS for Parents (Appendix B) is a subjective burden survey that was derived from the Informal Caregiver Survey (ICS) of the 1982 Long-term Care Survey sponsored by the Department of Health and Human Services. This questionnaire was used to measure subjective burden in caregivers of disabled persons and to capture dimensions of burden and benefits (Cafferta \& Stone, 1989). This survey was selected for inclusion in this study because the caregiver behaviors on the survey were similar to the behaviors that parents might use with a child taking prescribed steroids. The questionnaire is comprised of 12 items with higher scores indicating higher burden. Guttman scaling technique was used to determine types of burden indicated by the 12 items. Two factors (with eigen values greater than 1.0) emerged, role strain and role conflict. Additional findings indicated that more women experience 'relatively high' role conflict than do men $(25.8 \mathrm{v} .15 .9 \%)$ and more 'relatively high' role strain than do men (34.3 v. $24.8 \%$ ) (Cafferta \& Stone).

There was one item on the original Informal Caregiver Survey (Cafferta \& Stone, 1989) that was inappropriate for parents of children. This item inquired about senility in the care recipient. The developers of the ICS were contacted and gave approval to revise and use the questionnaire with this study sample of parents. The item about senility was deleted and replaced with an item regarding sleep, and the language for all items was changed to match the stem used in the CMCC (Keegan-Wells et al., 2002) by beginning each item with "My Child". The original survey had several items about multiple 
Table 3-1: Internal Consistency Estimates for Physical Care (PC) of the Care of My Child with Cancer Instrument.

\begin{tabular}{lll}
\hline & \multicolumn{2}{l}{ Standardized Variables } \\
\cline { 2 - 3 } $\begin{array}{l}\text { Deleted } \\
\text { Variable }\end{array}$ & $\begin{array}{l}\text { Correlation } \\
\text { with Total }\end{array}$ & Alpha \\
\hline Physical Care 1 & 0.96 & 0.96 \\
Physical Care 2 & 0.96 & 0.96 \\
Physical Care 3 & 0.96 & 0.96 \\
Physical Care 4 & 0.96 & 0.96 \\
Physical Care 5 & 0.96 & 0.96 \\
Physical Care 6 & 0.96 & 0.96 \\
\hline
\end{tabular}

Table 3-2: Internal Consistency Estimates for Emotional Care (EC) of the Care of My Child with Cancer Instrument.

\begin{tabular}{lll}
\hline & \multicolumn{2}{l}{ Standardized Variables } \\
\cline { 2 - 3 } $\begin{array}{l}\text { Deleted } \\
\text { Variable }\end{array}$ & $\begin{array}{l}\text { Correlation } \\
\text { with Total }\end{array}$ & Alpha \\
\hline Emotional Care 14 & 0.78 & 0.89 \\
Emotional Care 15 & 0.77 & 0.89 \\
Emotional Care 16 & 0.78 & 0.89 \\
Emotional Care 17 & 0.68 & 0.90 \\
Emotional Care 18 & 0.74 & 0.90 \\
Emotional Care 19 & 0.72 & 0.90 \\
Emotional Care 20 & 0.64 & 0.91 \\
\hline
\end{tabular}


symptoms within an item. An example of this type of item was, "My child becomes confused or refuses to cooperate." This item does not give the participant an option of selecting or not selecting one or both of the characteristics. These variables were separated, thus extending the number of items in the revised form to 17 (Appendix B). The Revised Informal Caregiver Survey (RICS) for Parents was scored on a Likert-like scale constructed from all items (17) with a range from 0 to 51 . (Never $=0$, Sometimes $=1$, Often=2, and Very Often=3). The higher score indicated a higher level of subjective burden.

A feasibility study was conducted (Tidwell, unpublished data) to estimate relevance (content validity) and clarity of the RICS for parents. Parents in the feasibility study were asked to complete the RICS and rate the clarity and relevancy of each question in the survey. (Appendix F) The study sample consisted of 8 mothers in the community who had children diagnosed with chronic illnesses. One child was diagnosed as having Attention Deficit Disorder (ADD) and the other seven children were diagnosed with asthma. All the parents identified their children as typically well even though their child was taking medication and described as needing regular treatments (Appendix $\mathbf{G}$ ). Total scores for subjective burden were relatively low. Only 2 out of 7 mothers indicated that taking care of their child was hard on them emotionally (Appendix H). The parents indicated that the items in the RICS were easy to understand and that the questions were mostly relevant (Appendix I and Appendix $\mathbf{J}$ ). No revisions of items and no additional questions were identified. One of the parents commented that the format was easy to read and to complete and that limiting the responses to 3 or 4 choices was very helpful and decreased haphazard response patterns. The average time needed to complete the questionnaire was about 4.49 minutes.

In this study the internal consistency estimates for items for the Revised Informal Caregiver Survey for Parents are shown in Table 3-3. Item number 16 ("I can't provide needed medical care for my child") was excluded from this table because all the primary caregivers' responses were the same ("never"). The items are reliable with standardized Cronbach alpha estimates ranging from 0.86 to 0.88 using a definition of reliability to be a Cronbach's alpha of greater than or equal to 0.70 (Litwin, 1995).

\section{$\underline{\text { Wrist Actigraph }}$}

Actigraphs and sleep diaries were used to evaluate sleep quality in the participants of this study. The actigraph used with the patients in the SLEEP study was the same model used with the parents in this study. The Mini Motionlogger AAM-32 (Ambulatory Monitoring Inc., Ardsley, NY) is a wristwatch style device that weighs 1 ounce and contains a biaxial piexoelectric sensor microprocessor with programmable epoch length. The epoch length determines data storage capacity; the epoch length for this study with parents was the very same as used with patients: 1 minute resulting in data storage for up to 16 days (a capacity that exceeded that needed for our 10-day study design). A scoring 
Table 3-3: Internal Consistency Estimates for the Revised Informal Caregiver Survey for Parents.

\begin{tabular}{lll}
\hline & \multicolumn{2}{l}{ Standardized Variables } \\
\cline { 2 - 3 } Deleted & $\begin{array}{l}\text { Correlation } \\
\text { vith Total }\end{array}$ & Alpha \\
\hline 1 Forgets & 0.22 & 0.88 \\
2 Confused & 0.52 & 0.87 \\
3 Cooperate & 0.35 & 0.88 \\
4 Upset & 0.67 & 0.86 \\
5 Yells & 0.46 & 0.87 \\
6 Embarrasses Me & 0.64 & 0.86 \\
7 Embarrasses Others & 0.55 & 0.86 \\
8 Free Time & 0.57 & 0.86 \\
9 Social Life & 0.60 & 0.86 \\
10 Privacy & 0.64 & 0.86 \\
11 Attention & 0.55 & 0.86 \\
12 Health & 0.64 & 0.86 \\
13 Feel Well & 0.40 & 0.87 \\
14 Emotion & 0.54 & 0.87 \\
15 Tasks & 0.43 & 0.87 \\
17 My Sleep & 0.39 & 0.88 \\
\hline
\end{tabular}

*Item \#16 was omitted from this analysis because all the responses were 0 (never). 
program accompanies this system and computes the following calculations: sleep onset, sleep duration, actual sleep minutes, number of awakenings, total sleep minutes, length of awakenings, sleep efficiency, and wake after sleep. Actigraph data were recorded for ten days. Parents were instructed on the use of the wrist actigraph during enrollment. Parent actigraph wrist bands were a different color than the bands being worn by their child who was enrolled on the SLEEP study to help the family members distinguish between the child and parent actigraphs.

Various studies have shown the actigraph to be a valid and reliable measure of activity and sleep in a variety of healthy and ill populations. Validation studies have compared simulateously obtained actigarphic and polysomnographic recordings. The findings have shown high correlations for actigraphic and polysomnographic whole-night sleep measures: duration $(\mathrm{r}=90)$ latency $(\mathrm{r}=.89)$ and wake after sleep onset $90.2 \%$ for healthy adult, 78.2 for insomniac and $85.7 \%$ for patients with sleep apnea (Carter 2003).

\section{Sleep Diary}

The Sleep Diary (Appendix C) is a 16-item self-report measure that allowed the parents to document their perceptions of their sleep and nap patterns during the previous 24-hour period. The items were derived from a sleep diary that was used by Dr. Van Brunt (Van Brunt et al., 1996) in his research with adults. Nap times, bedtimes, timeout- of bed, sleep interruptions and an overall rating of how the parents perceived their sleep was recorded on the sleep diary. Instructions on how to complete the sleep diary were given to the parents (Appendix $\mathbf{J}$ ).

Rodgers et al. (1993) conducted a study on the reliability of sleep diaries of assessment of sleep/wake patterns. Data between polysomnographic information and data recorded in sleep diaries were compared. The analysis was primarily descriptive. Cohen's kappa was used to measure agreement. Sensitivity and specificity were determined to provide information regarding the accuracy of the subjective information. The percentage agreement between the sleep diary and polysomnography was high (kappa=0.87).

\section{Brief Fatigue Inventory (BFI)}

The (BFI) (Appendix D-1) (Mendoza et al., 1999) was used to measure parental fatigue. A review of literature was conducted to evaluate and select an appropriate measure of parental fatigue, specifically an instrument that could possibly measure fatigue over time. The BFI is a 9-item self-report instrument that assesses the severity of fatigue and impact of fatigue on daily functioning on a $0-10$ numeric rating scale, with 0 meaning "no fatigue," and 10 meaning fatigue "as bad as you can imagine". Three items ask patients to rate the severity of their fatigue and the remaining six items assess how fatigue has interfered with different aspects of the patient's life during the past 24 hours. These items include general mood, walking ability, normal work, relations with other 
people, and enjoyment of life. The items that relate how fatigue interferes with daily living are also measured on a 0-10 scale, 0 indicating fatigue "does not interfere," with aspects of daily living and 10 meaning that fatigue "completely interferes" with aspects of daily living (Mendoza et al.).

In a study examining fatigue in patients with resectable rectal cancer receiving chemoradiation (Wang et al., 2001), patients who rate their "fatigue at its worst" (7 or greater) demonstrated that they had severe fatigue that impaired function. When rated at a level of 4 to 6 , patients were considered to have moderate levels of fatigue. The scoring of the BFI scale is obtained by averaging all the 9 items; each item is scored on a scale of 0 to 10 (integer values). The mean of the nine items was used as a global BFI score. The time required to complete this inventory is approximately 5 minutes (Mendoza et al., 1999).

The BFI was developed for use in clinical screenings and clinical trials. This instrument has been used in adult cancer patients and in community dwelling adults (Anderson et al., 2003). The BFI was shown to be an internally consistent measure of severity of fatigue. The Cronbach's alpha reliability ranges from 0.82 to 0.97 . (Mendoza et al., 1999). Concurrent validity was assessed by correlating the BFI with the Fatigue subscale of the Functional Assessment of Cancer Therapy-Fatigue (FACT) (Yellen et al., 1997) and the Vigor and Fatigue subscales of the Profile of Mood States (POMS) (McNair et al., 1971).

Participants in this study were asked to complete this instrument twice during each 5-day period. Internal consistency estimates for BFI measurements are shown in Table 3-4. The standardized Cronbach's alpha coefficients ranged from 0.83 to 0.88 . The BFI scores for the different days were reliable using a definition of Cronbach alpha estimate of greater than 0.70 .

\section{Procedure}

Research participants were asked to complete the demographic sheet, a fatigue measure and the caregiver burden instrument, and to keep a sleep diary and to wear a wrist actigraph to measure sleep characteristics. Parents were trained at the time of study enrollment about the use of the actigraph and they were also instructed on how to complete each of the study instruments. The parents/guardians were given a study packet that included an information letter, (Appendix K), a demographic form (Appendix E), instruction sheets (Appendix L), four copies of the Brief Fatigue Survey (Appendix D) (Mendoza et al., 1999), two copies of the Care of My Child with Cancer Instrument (Appendix A) (Keegan-Wells et al., 2002), two copies of the Revised Informal Caregiver's Survey for Parents (Appendix B), and sleep diary forms (Appendix C). These forms were color coordinated to match the time periods in which the forms were to be completed. Forms that were to be completed during the first 5-day period were printed on yellow paper and the forms that were to be completed during the second 5-day 
Table 3-4: Internal Consistency Estimates for Brief Fatigue Inventory Measurements.

\begin{tabular}{lll}
\hline & \multicolumn{2}{l}{ Standardized Variables } \\
\cline { 2 - 3 } BFI Score & $\begin{array}{l}\text { Correlation } \\
\text { with Total }\end{array}$ & Alpha \\
\hline Week 1 Day 2 & 0.85 & 0.83 \\
Week 1 Day 5 & 0.77 & 0.86 \\
Week 2 Day 2 & 0.73 & 0.88 \\
Week 2 Day 5 & 0.71 & 0.88 \\
\hline
\end{tabular}


period were printed on lavender paper. A time line (Appendix $\mathbf{M}$ ) was also included in the packet and instructions for using the wrist actigraph.

The data collection period included 10 consecutive days; 5 days before the dexamethasone started (week 1) and 5 days during dexamethasone therapy (week 2). Parents completed the BFI on days 2 and 5 before the child began the dexamethasone therapy and on days 2 and 5 during their child's dexamethasone therapy. Parents completed the sleep diary on days 2 and 5 before the child begins dexamethasone therapy and on days 2 and 5 during the child's dexamethasone therapy. The CMCC Instrument (Keegan-Wells et al., 2002) and the Revised Informal Caregiver Survey for Parents were completed on day 5 before dexamethasone (week 1) and on day 5 during the time the child was receiving dexamethasone (week 2). The parents wore the actigraph during the entire 10-day period of the study. With the permission of the research participants, we contacted the parents/guardians via telephone to remind them of data collection times. The research participants were given a $\$ 25.00$ meal or Wal-Mart certificate for participating in the study. No long-term evaluations were conducted in this study.

\section{Data Analysis}

\section{$\underline{\text { Specific Aim One - Parent Sleep }}$}

To compare parent sleep quality (sleep efficiency, actual sleep time, sleep duration, nocturnal awakenings and wake after sleep onset score (WASO)) during two consecutive 5-day periods when their child is off dexamethasone (week 1) treatment for ALL versus on dexamethasone (week 2). Sleep quality measured by wrist actigraphy was the primary indicator and a parent-report sleep diary was the additional subjective measure.

Related hypothesis one: Average (sleep quality) scores for parental sleep before dexamethasone (week 1) will be better (greater sleep time and sleep duration; less nocturnal awakenings, sleep efficiency and WASO) than average sleep quality scores during dexamethasone (week 2).

Sleep variables (sleep efficiency, actual sleep time, sleep duration, nocturnal awakenings and wake after sleep onset (WASO)) were obtained from the actigraph data for each day (corroborated by the sleep diary). Descriptive summary statistics for the average sleep variables (sleep efficiency, actual sleep time, sleep duration, nocturnal awakenings and wake after sleep onset (WASO)) between week 2 (on dexamethasone) and week 1 (off dexamethasone) were also calculated. An average for each of the two 5day time periods off (week 1) and on (week 2) dexamethasone was calculated. The means for the sleep variables during the off dexamethasone period and during the on dexamethasone period was calculated for each participant. Then, the difference between the two five-day periods was obtained and a one-sided, one sample student's t-test was used to test if the mean difference was significantly different from 0 . 
Mixed effects models using PROC MIXED in the statistical software package of SAS were used to model the relationships between outcome measures, such as efficiency scores and sleep duration, as a function of time week 1 (off dexamethasone) and week 2 (on dexamethasone).

$\underline{\text { Specific Aim Two - Parent and Child Sleep }}$

To assess the relationship between the parent and patient sleep quality during two consecutive 5-day periods, week 1 (off dexamethasone) versus week 2 (on dexamethasone) as measured by wrist actigraph.

Related hypothesis two: Average sleep quality scores (sleep efficiency, actual sleep time, sleep duration, nocturnal awakenings, and wake after sleep onset score (WASO)) before dexamethasone (week 1) will be better (greater sleep time, and sleep duration, less nocturnal awakenings, sleep efficiency, and WASO) than average sleep scores during dexamethasone (week 2). Parental and child sleep scores will have a directional relationship to one another

Mixed effects models methods using PROC MIXED in the statistical software package of SAS, as described above, was used to model differences (between week 1 and week 2) in the sleep variables over each 5-day period for caregivers as a function of similar differences for child and time. A significant $p$-value (0.05) corresponding to the coefficient of child's sleep variables was considered to be indicative of a significant association for the parent and child scores.

\section{$\underline{\text { Specific Aim Three - Parent Fatigue }}$}

To compare parent fatigue as measured by the Brief Fatigue Inventory (BFI) during the two consecutive 5-day periods of their child being off (week 1) versus on (week 2) dexamethasone.

Related hypothesis three: Parent fatigue scores will be less before dexamethasone (week 1) than during dexamethasone (week 2).

Caregivers completed the BFI on day 2 and day 5 of each of the two study periods. To assess the impact of dexamethasone on fatigue of the research participants, an analysis of the average BFI scores on day 2 and 5 for each of the two time periods (week 1 (off dexamethasone) versus week 2 (on) dexamethasone) were calculated and then the differences of the averages were examined; if the difference was significantly different from 0 using a one-sample t-test, the change in fatigue was considered to be statistically significant. An analysis using the PROC MIXED method was also performed taking into account the longitudinal nature of the study design. 


\section{$\underline{\text { Specific Aim Four - Parent Caregiver Burden }}$}

To compare parental caregiver burden (time and effort related to care responsibilities) during the two consecutive 5-day periods as measured by the Revised Caregiver Survey (subjective measure) and the Care of My Child with Cancer Instrument (objective measure).

Related hypothesis four: Parental subjective and objective burden scores will be greater during dexamethasone (week 2) than before dexamethasone (week 1).

Scoring for the CMCC instrument combined both time and effort to the demand associated with each caregiving task. Individual item demand scores were computed by multiplying the time score by the effort for each item and then we took the square root of their product to return to the demand score of the original metric (scores ranging from 1 to 5). The total demand score was summed across all items. Item demand scores were not computed if no response was indicated for either time or effort for a given item. The higher demand score indicated a higher level of objective burden. The Revised Informal Caregiver Survey (RICS) for Parents was scored on a Likert-like scale constructed from all the items (17) with a range from 0-51. (Never=0, Sometimes=1, Often $=2$, and Very Often=3). The higher score indicated a higher level of subjective burden.

The RICS for parents and the CMCC were both completed on Day 5 of each of the two 5-day study periods. The research participants had subjective and objective caregiver burden scores for each of the two time periods. Comparison between objective and subjective caregiver burdens for the two time periods was not done because of missing information in the $\mathrm{CMCC}$ instrument.

\section{Specific Aim Five - Relationships among Sleep, Fatigue, and Caregiver Burden}

To assess the relationships among parent sleep quality, fatigue, and caregiver burden within each of the consecutive 5-day periods.

Related hypothesis five: The relationship between parental fatigue would be inversely related to parent sleep and directly related to parent caregiver burden. Average parental scores for sleep quality, fatigue, and caregiver burden before dexamethasone (week 1) will be less than average scores during dexamethasone (week 2).

The mixed effect models method from PROC MIXED in the SAS statistical software package was used to assess the relationships among caregiver burden, sleep quality, and fatigue during off (week 1) and on (week 2) dexamethasone periods using the actigraph data and the caregiver self-report data. In a similar manner, differences between the two periods on the outcome measures of interest were obtained and the regression model technique was used to assess the relationships with other covariates of interest such as the child's fatigue score. 
$\underline{\text { Specific Aim Six - Parent Sleep, Fatigue, Caregiver Burden, and Demographic Variables }}$

To explore if parent sleep quality, fatigue, and caregiver burden differs by parent age, marital status, ethnicity, child's risk category (low versus standard), or the child's age.

The mixed effect models technique from PROC MIXED in the SAS statistical software package was used to assess associations among age, marital status, ethnicity, child's risk category or child's age and parent sleep, fatigue, and caregiver burden scores. In the model, the dependent variables (sleep efficiency, actual sleep time, nocturnal awakenings, sleep duration, WASO fatigue, and caregiver burden) were regressed on the independent predictors (marital status, age, ethnicity, child's risk category or child's age).

\section{Human Subjects Consideration}

Obtaining Informed Consent

Parents or guardians of patients who were enrolled on SJCRH Total XV and whose child enrolled on the SLEEP protocol (continuation phase and approaching week 50) were eligible to participate in this study. The research participants for this study were approached after their child had been enrolled in the SLEEP study. Parents were enrolled in this study only after they were fully informed about the study in a face-to-face conversation (Appendix N). Parents had the option to decline, participate, or withdraw after enrollment at any time. Reasons for refusals were documented and included in the annual reports to the institutional review board (IRB) (Appendix O). All parents who met the eligibility criteria were fully welcome to participate in this study.

\section{$\underline{\text { Staff Training and Integrity of Intervention }}$}

Study team members participated in a formal training session (Appendix $\mathbf{P}$ ) that was conducted before the study began. Consistency in participant accrual and consent processes was ensured so that the study would be completed in a timely and efficient manner. Only team members who completed the interview training process were allowed to participate in interviews or informed consent processes.

\section{Criteria for Removal from Protocol and Off-Study Criteria}

The removal of the wrist actigraph after the 10-day study period ended participation in this study. The research participants returned the completed study forms and wrist actigraph. In addition, if the parent's child was hospitalized during the data collection period, the parent was to be considered ineligible or as off-study. However, this did not occur during the study. 


\section{Safety and Adverse Event Reporting Requirements}

In addition to the continuing review reports to the St. Jude Children's Research Hospital Institutional Review Board (IRB) (Appendix P), the Principle Investigators of this study were responsible for reporting all serious or unexpected adverse events that could impact the safety of or risk experienced by the parents. Unexpected distress was to be reported to the SJCRH IRB office immediately. Per St. Jude Children's Research Hospital Institutional Review Board, serious, unexpected events were to be reported within 48 hours, and all others within 10 working days. No adverse events occurred during this study. SJCRH IRB approval and reporting requirements were reviewed and accepted by University of Tennessee Health Science Center's Institutional Review Board.

\section{Data Collection and Confidentiality}

The research participants were provided with self-addressed envelopes to mail back the completed study instruments. Participants were also provided with addressed Federal Express boxes to return the actigraph. If the research participant preferred, they were contacted via telephone to remind them of data collection time points. Study numbers were used in place of an identifier such as a medical record number. No patient or parent names were recorded on the data collection forms. The data were maintained in a locked file and will be destroyed after all data have been analyzed and study findings fully disseminated. 


\section{CHAPTER 4: RESULTS}

The purpose of the present study of parent caregivers of children with ALL was to evaluate parent sleep, fatigue, and caregiver burden immediately before and during their child's dexamethasone pulse during continuation therapy for ALL and to compare parent quality of sleep and fatigue with their child's sleep. Findings related to the first specific aim address how parent sleep patterns differ between the two time periods (off dexamethasone (week 1) and during dexamethasone (week 2)). Additional findings related to the second specific aim will address the relationship between parent and child sleep patterns. In this chapter, each research aim is addressed with results reported by the following sub-categories: primary caregivers $(n=17)$, mothers $(n=15)$, fathers $(n=5)$, and couples $(n=4)$. Results for the differences between sleep quality scores, fatigue scores, and caregiver burden scores for the two consecutive 5-day periods are only reported for primary caregivers and mothers. Tables displaying results for mothers, fathers, and couples are located in the appendix.

\section{Primary Caregiver Characteristics}

Seventeen primary caregivers (15 mothers and 2 fathers) participated in this study. The majority of primary caregivers were white, female, and homemakers at the time of the study. Their average age was 38 years $(S D=6)$, and most of the primary caregivers had other children in addition to their sick child. The majority of the primary caregivers (88\%) had at least a high school diploma and 53\% attended college. The ill child risk group classification was evenly distributed between low risk (53\%) (patients that present with characteristics associated with good prognosis at diagnosis) and standard risk (47\%) (patients that present with characteristics associated with poor prognosis at diagnosis). The demographic information for the primary caregivers is shown in Table 4-1 below.

Seventeen mothers were consented to participate in this study. One of the mothers did not complete study forms, one mother withdrew from the study, and one mother was withdrawn by the study committee due to study enrollment closure. One child's actigraph data malfunctioned, thus leaving one primary caregiver (a mother) inevaluable for specific aim two (comparison of parent and child sleep quality) and one mother's actigraphy data were insufficient to analyze and therefore inevaluable for specific aim one (comparison of parent sleep quality). A total of thirteen mothers had sufficient actigraph data for analysis.

Fathers also had the opportunity to participate in this study. Seven fathers were enrolled on the study, with five being evaluable for actigraph analysis, which is defined as having actigraph data for at least 18 hours on 3 out of the 5 days for each time period. Of the two fathers whose data were not evaluated, one father did not complete the study questionnaires during the second 5-day period (week 2) and did not wear the actigraph 
Table 4-1: Demographic Information for Primary Caregivers $(n=17)$.

\begin{tabular}{|c|c|c|c|}
\hline Characteristic & $\mathrm{n}(\%)$ & Characteristic & $\mathrm{n}(\%)$ \\
\hline Relationship & & Primary Occupation & \\
\hline Father & $15(88)$ & Homemaker & $6(35)$ \\
\hline \multirow[t]{2}{*}{ Mother } & $2(12)$ & Unemployed & $3(18)$ \\
\hline & & Official and Managers & $2(11)$ \\
\hline Marital Status & & Other & $3(18)$ \\
\hline Married & $12(71)$ & Missing Data & $3(18)$ \\
\hline Single/Divorced & $5(29)$ & & \\
\hline Race & & Number of Dependents & \\
\hline White & $14(82)$ & $1-2$ & $8(47)$ \\
\hline Black & $2(12)$ & $3-4$ & $8(47)$ \\
\hline Other & $1(6)$ & 5 or More & $1(6)$ \\
\hline Age (years) & & Annual Income & \\
\hline Mean (Standard Deviation) $\backslash$ & $38(6)$ & Less Than 20000 & $5(29)$ \\
\hline \multirow[t]{3}{*}{ Median (Range) } & $39(28-47)$ & $20000-40000$ & $3(18)$ \\
\hline & & Over 40000 & $8(47)$ \\
\hline & & Missing Data & $1(6)$ \\
\hline \multicolumn{4}{|l|}{ Education } \\
\hline Less Than High School & $2(12)$ & & \\
\hline High School Graduate & $6(35)$ & & \\
\hline College & $7(41)$ & & \\
\hline Post College & $2(12)$ & & \\
\hline
\end{tabular}


the total ten days, and one father's actigraph data were insufficient to analyze. Two of the five evaluable fathers were primary caregivers.

\section{Specific Aim One - Parent Sleep}

To compare parent sleep quality (sleep efficiency, actual sleep time, sleep duration, nocturnal awaking, and wake after sleep onset score (WASO)) and sleep duration during two consecutive 5-day periods (when their child being treated for ALL is off (week 1) versus on (week 2) dexamethasone) as measured by wrist actigraph (primary indicator) and a sleep diary (subjective measure).

Related hypothesis one: Average sleep quality scores for parental sleep before dexamethasone (week 1) will be better (greater sleep time and sleep duration, less nocturnal awakenings, sleep efficiency and WASO) than average sleep quality scores during dexamethasone (week 2).

\section{Findings for Primary Caregivers}

\section{Actigraph Results for Primary Caregivers}

There were a total of 17 primary caregivers in the study; actigraph data were available for 15 primary caregivers. When calculating the average scores for comparing study variables between the two 5-day study periods, only the patients who had at least three of the five days of sleep data in each study period were included in the data analysis. Descriptive statistics for average sleep efficiency scores, actual sleep time, nocturnal awakenings, sleep duration and WASO during two consecutive 5-day periods of off versus on dexamethasone (week 1 versus week 2) for actigraphy are shown in Table 4-2. The average sleep efficiency scores, nocturnal awakenings and WASO were higher for the primary caregivers when the child was on dexamethasone compared to when they were off dexamethasone. Primary caregivers had a significantly higher actual sleep time and sleep duration when their children were on dexamethasone compared to when they were off dexamethasone (Table 4-3).

Actigraphy results from the Longitudinal Mixed Effect Model technique using PROC Mix are presented in Table 4-4. For this analysis, week 2 was coded as baseline. Primary caregivers had significantly higher actual sleep time and sleep duration when their children were on dexamethasone compared to when they were off dexamethasone. PROC Mixed Model analysis was completed to correct for correlation among the observations. Each of the sleep variables was used as response (dependent) variables and the week (exposure to dexamethasone) was used as the independent variable in the model. 
Table 4-2: Descriptive Sleep Quality Results by Actigraph for Primary Caregivers $(n=15)$.

\begin{tabular}{|c|c|c|c|c|c|c|}
\hline Sleep Variables & Week & Mean & $\mathrm{SD}$ & Mdn & Min & Max \\
\hline \multirow[t]{2}{*}{ Efficiency Scores } & 1 & 73.47 & 15.87 & 81.29 & 45.01 & 90.52 \\
\hline & 2 & 75.66 & 14.75 & 74.59 & 38.65 & 92.22 \\
\hline \multirow[t]{2}{*}{ Actual Sleep Time } & 1 & 330.79 & 84.25 & 335.60 & 182.00 & 466.60 \\
\hline & 2 & 391.04 & 95.53 & 392.75 & 208.67 & 528.00 \\
\hline \multirow[t]{2}{*}{ Sleep Duration } & 1 & 476.28 & 53.79 & 472.20 & 369.60 & 547.40 \\
\hline & 2 & 558.84 & 122.01 & 537.80 & 422.40 & 866.67 \\
\hline \multirow[t]{2}{*}{ Nocturnal Awakenings } & 1 & 14.05 & 5.11 & 15.20 & 5.00 & 21.75 \\
\hline & 2 & 14.33 & 7.00 & 16.00 & 1.33 & 25.00 \\
\hline \multirow[t]{2}{*}{ WASO Scores } & 1 & 119.72 & 71.57 & 86.60 & 44.80 & 260.60 \\
\hline & 2 & 124.74 & 74.77 & 106.25 & 43.60 & 334.00 \\
\hline
\end{tabular}


Table 4-3: Differences in Average Sleep Quality Results between Week 2 and Week 1 for Primary Caregivers by Actigraphy $(n=15)$.

\begin{tabular}{lllllllc}
\hline Sleep Variables & Mean & SD & Mdn & Min & Max & $t$ & $p$ \\
\hline Efficiency Scores & 2.19 & 12.55 & -0.89 & -11.43 & 35.31 & 0.68 & 0.51 \\
Actual Sleep Time & 60.25 & 91.33 & 34.60 & -25.25 & 241.50 & 2.56 & $0.02^{*}$ \\
Sleep Duration & 82.56 & 101.01 & 77.40 & -82.80 & 319.66 & 3.17 & $0.01^{*}$ \\
Nocturnal & 0.28 & 5.10 & 0.40 & -9.92 & 10.00 & 0.21 & 0.84 \\
Awakenings & & & & & & & \\
WASO Scores & 5.02 & 59.62 & 3.40 & -117.42 & 91.80 & 0.33 & 0.75 \\
\hline
\end{tabular}

Table 4-4: Summary of Longitudinal Proc Mixed Regression Model Findings for the Primary Caregivers' Actigraphy Scores.

\begin{tabular}{llll}
\hline Response Variable & Covariate & Coefficient & P-value \\
\hline Sleep Efficiency & Week & -1.6 & 0.50 \\
Actual Sleep Time & Week & -57.6 & $0.024^{*}$ \\
Sleep Duration & Week & -76.7 & $0.003^{*}$ \\
Nocturnal Awakenings & Week & -0.4 & 0.71 \\
WASO & Week & -7.3 & 0.58 \\
${ }^{*} p=<0.05$ & & &
\end{tabular}




\section{Sleep Diary Results for Primary Caregivers}

The Sleep Diary instrument was based on self-report. The report was documented on day 2 and day 5 on and off dexamethasone. Averages of sleep duration by diary report for primary caregivers over time are given in Table 4-5. Notably, parents reported their sleep time was about the same when their children were on (week 2) or off (week 1) dexamethasone. Sleep duration by sleep diary report was not statistically different between week 2 (on dexamethasone) and week 1 (off dexamethasone) ( $p=0.70)$, indicating that parents do not perceive changes in their sleep duration.

The sleep duration information from the Sleep Diary was compared with the actigraph data. Descriptive statistics for sleep duration by diary report and actigraph are shown in Table 4-5. When their child was off dexamethasone, the primary caregivers reported longer sleep duration compared to what was recorded by the actigraph. Sleep duration reported by sleep diary report for week 1 day 2 was significantly higher compared to those recorded by actigraph on week 1 day 2 . However, the results were not consistent during week 2. Parents reported less sleep duration by sleep diary report for week 2 day 2 than recorded by actigraph; during week 2 day 5 parent sleep duration by actigraph recordings were less than parent sleep diary reports. Differences in sleep duration between actigraph and diary report are shown in Table 4-6. Sleep duration reported by sleep diary report for week 1 day 2 was significantly higher compared to those recorded by actigraph.

Additional sleep quality measurements assessed by the parent diary report are included in Table 4-7. The numbers of primary caregivers who completed the sleep diary was different for some days. Overall, the number of primary caregivers that completed the sleep diary report declined by approximately $50 \%$ by day 5 of week 2 with the exception of the questions that were asked once in each time period. Descriptively, more primary caregivers reported taking naps on day 2 and day 5 when the child was on dexamethasone. More parents reported participating in exercise during week 1 than in week 2 . Primary caregivers also reported more caffeine consumption during week 1 than in week 2.

Primary caregivers were also asked if their sleep was interrupted by their child with cancer. A Likert scale of 0 to $4(0=$ not at all to $4=$ a great deal $)$ was used to measure this interruption. Because so few parents reported interrupted sleep due to their ill child, percentages were calculated using the number of times the response of 0 (meaning not at all) was recorded over the total number of recorded responses.

Primary caregivers were asked to rate their sleep quality using the sleep diary (Appendix C). A Likert scale of 1 to 4 ( $1=$ exhausted, $2=$ tired, $3=$ average, and $4=$ refreshed) was used for reporting how they felt when they woke up. Likewise a Likert scale of 1 to 4 ( $1=$ very restless, $2=$ restless, $3=$ average quality, and $4=$ very sound) was used to report overall sleep quality. The sleep quality proportions are summarized in Table 4-8. Half the primary caregivers reported having been exhausted or refreshed 
Table 4-5: Sleep Duration Scores by Actigraph and Sleep Diary Report for Primary Caregivers.

\begin{tabular}{llllllll}
\hline Instrument & Time & $\mathrm{n}^{*}$ & Mean & SD & Mdn & Min & Max \\
\hline Sleep Diary & Week 1-Day 2 & 14 & 560.71 & 110.85 & 530.00 & 420.00 & 780.00 \\
& Week 1-Day 5 & 12 & 507.92 & 123.98 & 450.00 & 390.00 & 755.00 \\
& Week 2-Day 2 & 13 & 500.00 & 99.69 & 480.00 & 375.00 & 750.00 \\
& Week 2-Day 5 & 10 & 506.67 & 76.85 & 532.50 & 405.00 & 575.00 \\
& & & & & & & \\
Actigraph & Week 1-Day 2 & 14 & 468.50 & 152.65 & 473.00 & 47.00 & 631.00 \\
& Week 1-Day 5 & 12 & 409.33 & 132.12 & 357.50 & 246.00 & 679.00 \\
& Week 2-Day 2 & 14 & 509.71 & 143.92 & 513.50 & 299.00 & 820.00 \\
& Week 2-Day 5 & 10 & 486.80 & 145.59 & 456.00 & 288.00 & 721.00 \\
\hline
\end{tabular}

* Note missing data from some days 
Table 4-6: Difference in Sleep Duration Results between Actigraph and Sleep Diary Reports for Primary Caregivers.

\begin{tabular}{|c|c|c|c|c|c|c|c|c|}
\hline $\begin{array}{l}\text { Diary- } \\
\text { Actigraph }\end{array}$ & $\mathrm{n}$ & Mean & $\mathrm{SD}$ & Mdn & Min & Max & $t$ & $p$ \\
\hline \multicolumn{9}{|l|}{ Week Day } \\
\hline Week 1-Day 2 & 14 & -92.214 & 136.309 & -80.5 & -373 & 108 & -2.531 & $0.025 *$ \\
\hline Week 1-Day 5 & 12 & -98.583 & 215.844 & -114.5 & -458 & 244 & -1.582 & 0.142 \\
\hline Week 2-Day 2 & 14 & 12.571 & 182.308 & -29.0 & -301 & 340 & 0.258 & 0.800 \\
\hline Week 2-Day 5 & 10 & 96.800 & 260.365 & 61.5 & -287 & 475 & 1.176 & 0.270 \\
\hline
\end{tabular}


Table 4-7: Summary of Sleep Diary Results for Primary Caregivers.

\begin{tabular}{|c|c|c|c|c|}
\hline Variables & $\begin{array}{l}\text { Week } 1 \\
\text { Day } 2\end{array}$ & $\begin{array}{l}\text { Week } 1 \\
\text { Day } 5\end{array}$ & $\begin{array}{l}\text { Week } 2 \\
\text { Day } 2\end{array}$ & $\begin{array}{l}\text { Week } 2 \\
\text { Day } 5\end{array}$ \\
\hline Naps & $0 / 14(0 \%)$ & $3 / 12(25 \%)$ & $3 / 13(23 \%)$ & $2 / 9(22 \%)$ \\
\hline Sleep Aids & None & None & None & $1 / 6(17 \%)$ \\
\hline $\begin{array}{l}\text { Exercises (Including } \\
\text { Vigorous House } \\
\text { Cleaning) }\end{array}$ & $9 / 12(75 \%)$ & $7 / 10(70 \%)$ & $7 / 12(58 \%)$ & $4 / 6(67 \%)$ \\
\hline Activity to Help Sleep & $5 / 11(46 \%)$ & $3 / 8(38 \%)$ & $4 / 11(37 \%)$ & $2 / 6(33 \%)$ \\
\hline $\begin{array}{l}\text { My Sleep Was } \\
\text { Interrupted by My Child } \\
\text { - Not At All/Total \# of } \\
\text { Responses }\end{array}$ & $11 / 13(85 \%)$ & $9 / 11(82 \%)$ & $8 / 12(67 \%)$ & $4 / 7(57 \%)$ \\
\hline $\begin{array}{l}\text { Did The Child Sleep } \\
\text { with Parent? } \\
\text { Yes/Total \# of } \\
\text { Responses }\end{array}$ & $5 / 14(36 \%)$ & $4 / 10(40 \%)$ & $6 / 13(46 \%)$ & $2 / 8(25 \%)$ \\
\hline $\begin{array}{l}\text { Consumed Beverage } \\
\text { with Caffeine }\end{array}$ & $*$ & $11 / 12(92 \%)$ & $*$ & $6 / 10(60 \%)$ \\
\hline $\begin{array}{l}\text { Food Consumed } \\
\text { Containing Caffeine }\end{array}$ & * & $8 / 12(66 \%)$ & * & $4 / 10(40 \%)$ \\
\hline $\begin{array}{l}\text { Amount of Alcohol } \\
\text { Consumed }\end{array}$ & $*$ & $2 / 10(20 \%)$ & $*$ & $1 / 9(11 \%)$ \\
\hline
\end{tabular}

\footnotetext{
* These questions were only asked on Day 5 of each week.
} 
Table 4-8: Summary of Subjective Sleep Quality for Primary Caregivers.

\begin{tabular}{lllll}
\hline & $\begin{array}{l}\text { Week 1 } \\
\text { Day 2 }\end{array}$ & $\begin{array}{l}\text { Week 1 } \\
\text { Day 5 }\end{array}$ & $\begin{array}{l}\text { Week 2 } \\
\text { Day 2 }\end{array}$ & $\begin{array}{l}\text { Week 2 } \\
\text { Day 5 }\end{array}$ \\
\hline $\begin{array}{l}\text { When I Woke Up This } \\
\text { Morning, I Felt } \\
\text { Exhausted (1) }\end{array}$ & & & & \\
Tired (2) & $2 / 14(14 \%)$ & $1 / 12(8 \%)$ & $1 / 13(7 \%)$ & $0 / 8(0 \%)$ \\
Average (3) & $5 / 14(36 \%)$ & $5 / 12(42 \%)$ & $5 / 13(39 \%)$ & $3 / 8(37 \%)$ \\
Refreshed (4) & $5 / 14(36 \%)$ & $3 / 12(25 \%)$ & $5 / 13(39 \%)$ & $5 / 8(63 \%)$ \\
& $2 / 14(14 \%)$ & $3 / 12(25 \%)$ & $2 / 13(15 \%)$ & $0 / 8(0 \%)$ \\
& & & & \\
Overall Sleep Quality & & & & \\
Very Restless (1) & $0 / 12(0 \%)$ & $1 / 10(10 \%)$ & $2 / 13(15 \%)$ & $3 / 8(38 \%)$ \\
Restless (2) & $5 / 12(42 \%)$ & $3 / 10(43 \%)$ & $3 / 13(23 \%)$ & $1 / 8(12 \%)$ \\
Average (3) & $3 / 12(25 \%)$ & $4 / 10(40 \%)$ & $3 / 13(23 \%)$ & $2 / 8(25 \%)$ \\
Sound (4) & $4 / 12(33 \%)$ & $2 / 10(20 \%)$ & $5 / 13(39 \%)$ & $2 / 8(25 \%)$ \\
\hline
\end{tabular}


when they woke up. Of the parents who reported on day 5 of week 2, more than half rated their sleep as average or refreshed.

\section{Findings for Mothers}

\section{Actigraph Results for Mothers}

There were a total of 15 mothers in the study; actigraph information for 13 mothers was available for analyses. When calculating the average scores for comparing sleep study variables between the two 5-day study periods, only the mothers who had at least three of the five days of sleep data were included in the data analysis.

Descriptive statistics for average sleep efficiency scores, sleep time, nocturnal awakenings, and WASO during two consecutive 5-day periods of off versus on dexamethasone (week1 versus week 2) are shown in Table Q-1. The mothers' average sleep time, nocturnal awakenings, and WASO were higher when their children were on dexamethasone compared to when they were off dexamethasone.

Differences in the average sleep efficiency scores, sleep time, nocturnal awakenings and WASO between week 2 and week1 are presented in Table Q-2. Mothers had a significantly higher actual sleep time and sleep duration when their children were on dexamethasone compared to when they were off dexamethasone. The results of the longitudinal mixed effect model analysis are presented in Table Q-3. For this analysis, week 2 was coded as baseline. Mothers had a significantly higher actual sleep time and sleep duration when their children were on dexamethasone compared to when they were off dexamethasone. These results did not support the hypothesis for this specific aim.

\section{$\underline{\text { Sleep Diary Results for Mothers }}$}

Averages of sleep duration by sleep diary report for mothers are shown in Table Q-4. There was no difference in sleep duration by diary report between week 1 and week 2. Sleep duration by diary report and actigraph are also shown in Table Q-4. When their child was off dexamethasone, the mothers reported longer sleep duration compared to what was recorded by the actigraph. These differences were statistically significant.

\section{Findings for Fathers}

\section{Actigraph Results for Fathers}

There were a total of 5 fathers in the study. Aggregate findings from fathers need to be interpreted with caution because only a few fathers were enrolled in the study. Actigraph results for fathers are shown in Table R-1. Fathers' actigraphy results 
included longer actual sleep time, higher efficiency scores and longer sleep duration in week two than week one. The average sleep duration time for fathers was 504 minutes in week 1 and 561 minutes in week 2.

\section{Sleep Diary Results for Fathers}

Sleep diary reports for the fathers in this study are shown in Table R-2. Fathers reported less sleep duration per sleep diary report than actigraph recording during week 2 , when their child was on dexamethasone. Sleep duration for fathers for week 1 day 2 by actigraph and sleep dairy report was 400.83 minutes and 488.25 minutes respectively. Fathers reported sleep duration by sleep diary report at 462.14 minutes during week 1 day 2 , while actigraphy recorded 517.40 minutes on the same day.

\section{Findings for Couples}

\section{Actigraph Results for Couples}

There were a total of four married couples in the study. Aggregate findings from couples need to be interpreted with caution because only a few couples completed the study. Mothers reported higher sleep duration and actual sleep time in both time periods (week 1 and week 2). Fathers actually had less sleep duration time in week 2 but greater actual sleep time as recorded by actigraphy. The descriptive statistics for the sleep variables for these four couples during week 1 and week 2 are shown in Table S-1.

\section{$\underline{\text { Sleep Diary Results for Couples }}$}

Sleep diary reports for couple in this study are shown in Table S-2. Father consistently reported less sleep duration than mothers in both time periods. Fathers average sleep duration report on week 1 day 5 was 396.67 minutes (6.6 hours). This was the lowest average sleep duration recorded in both time periods for couples. Mothers average sleep duration report was highest on week 1 day 2 at 618.33 (10.3 hours). Both mothers and fathers recorded getting the most sleep on day 2 of week 1 .

\section{Specific Aim Two - Parent and Child Sleep}

Assess the relationship between the parent and child sleep quality during the two consecutive 5-day periods, when the child is off (week 1) versus on (week 2) dexamethasone, as measured by wrist actigraph.

Related hypothesis two: Average sleep quality scores (sleep efficiency, actual sleep time, sleep duration, nocturnal awakenings, and wake after sleep onset score 
(WASO)) before dexamethasone (week 1) will be better (greater sleep time and sleep duration, less nocturnal awakenings, sleep efficiency, and WASO) than average sleep quality scores during dexamethasone (week 2). Parental and child sleep scores will have a directional relationship to one another

\section{Findings for Primary Caregivers and Child Sleep Variables}

The total number of parent/child dyads for this analysis is 14 based on the availability of complete actigraph data for each dyad. Descriptive statistics for average of sleep efficiency, actual sleep time, sleep duration, nocturnal awakenings, and WASO for matched parent and child are shown in Table 4-9. Sleep efficiency scores for primary caregivers and their children were very similar for both week 1 and week 2 . The average sleep efficiency scores for parents were 76.81 week 1 and 75.54 for week 2; and average sleep efficiency scores for the children were 78.82 for week 1 and 79.94 for week 2 . Desired sleep efficiency scores for healthy adults is $85 \%$ or higher per night (DSM-IVTR, 2000).

Overall, primary caregivers had less actual sleep time than their child. Primary caregivers slept an average of 345.41 minutes ( 5.75 hours) during week 1 and 376.05 minutes (6.26 hours) during week 2 . Normal actual sleep time (total minutes of night time sleep) for adults is 6 to 8 hours per night (DSM-IV-TR, 2000).

Primary caregivers and their children alike slept longer during week 2 (when the child was on dexamethasone). Sleep duration as recorded by actigraphy was longer than sleep duration reported by primary caregiver sleep diary report. Sleep duration recordings for primary caregivers for week 1 and week 2 were 475.40 minutes ( 7.9 hours) and 544.91 minutes ( 9 hours) respectively. Average sleep duration for adults aged 35-50 ranged from 5.4 to 7.8 hours (Ohayon et al., 2004). Average sleep duration for children is 10 to 11 hours per night (Grigg-Damberger, 2004). Sleep duration for the children in this current study was 495.63 minutes (8.26 hours) for week 1 and 550.69 minutes $(9.17$ hours) in week 2.

The number of nocturnal awakenings was also similar for the parent/child dyad. Notably, the average number of night awakenings for the children during week 1 was more than that of their parents. The average number of nocturnal awakenings for the primary caregiver/child dyad ranged from 14 to 16 times for both time periods. Healthy children usually awaken one to five times a night (Riter \& Willis, 2004) while adults usually awaken 1 to 2 times per night (Mindell \& Durand, 1992).

Wake after sleep onset scores (WASO) were consistently higher for primary caregivers than for their ill child. Desired WASO for adults is 30 minutes or less per night (DSM-IV-TR, 2000). Primary caregivers WASO scores for week 1 and week 2 were 106.08 and 124.54 respectively. 
Table 4-9: Sleep Quality Results for Matched Primary Caregiver and Child by Actigraphy $(\mathrm{n}=14)$.

\begin{tabular}{|c|c|c|c|c|c|c|c|}
\hline Sleep Variable & Parent/Child & Week & Mean & SD & Mdn & Min & Max \\
\hline \multirow[t]{4}{*}{ Efficiency Score } & Primary Care Giver & 1 & 76.81 & 14.27 & 85.08 & 45.01 & 90.52 \\
\hline & & 2 & 75.54 & 15.62 & 79.34 & 38.65 & 92.22 \\
\hline & Child & 1 & 78.82 & 16.57 & 81.15 & 40.93 & 98.26 \\
\hline & & 2 & 79.94 & 14.49 & 84.38 & 47.94 & 96.97 \\
\hline \multirow[t]{4}{*}{ Actual Sleep } & Primary Care Giver & 1 & 345.41 & 77.54 & 348.20 & 205.00 & 466.60 \\
\hline & & 2 & 376.05 & 96.11 & 376.93 & 208.67 & 528.00 \\
\hline & Child & 1 & 377.44 & 115.01 & 399.00 & 153.40 & 533.60 \\
\hline & & 2 & 425.21 & 145.30 & 440.00 & 120.00 & 641.40 \\
\hline \multirow[t]{4}{*}{ Sleep Duration } & Primary Care Giver & 1 & 475.40 & 56.18 & 475.80 & 369.60 & 547.40 \\
\hline & & 2 & 544.91 & 117.90 & 529.20 & 422.40 & 866.67 \\
\hline & Child & 1 & 495.63 & 46.45 & 502.80 & 401.60 & 553.00 \\
\hline & & 2 & 550.69 & 94.91 & 539.00 & 333.00 & 688.60 \\
\hline Number of Nocturnal & Primary Care Giver & 1 & 14.37 & 4.57 & 16.00 & 5.00 & 19.40 \\
\hline \multirow[t]{3}{*}{ Awakenings } & & 2 & 14.59 & 6.79 & 15.01 & 1.33 & 25.00 \\
\hline & Child & 1 & 16.34 & 7.29 & 15.30 & 3.80 & 26.40 \\
\hline & & 2 & 14.20 & 6.18 & 12.17 & 5.25 & 23.80 \\
\hline \multirow[t]{4}{*}{ WASO Score } & Primary Care Giver & 1 & 106.08 & 67.07 & 71.80 & 44.80 & 260.60 \\
\hline & & 2 & 124.54 & 77.23 & 112.10 & 43.60 & 334.00 \\
\hline & Child & 1 & 95.69 & 67.32 & 91.75 & 19.40 & 248.40 \\
\hline & & 2 & 104.16 & 68.79 & 90.00 & 24.00 & 263.20 \\
\hline
\end{tabular}


Results from the Longitudinal mixed effect model using PROC MIXED are presented in Table 4-10. Primary caregiver sleep variables were used as dependent variables in the analysis and child sleep variables and week were used as covariates (independent variables). For this analysis, week 2 was coded as baseline. Adjusting for the child's sleep variables, this test was conducted to see if primary caregiver's sleep variables would change during week 1 and week 2 . Primary caregivers had a significantly lower actual sleep time and sleep duration compared to child's actual sleep time and sleep duration. Actual sleep time for the primary caregivers and their children were significantly higher when on dexamethasone compared to when off dexamethasone (Table 4-10). There were positive associations between primary caregiver and child actual sleep time and sleep duration. These results partially support the hypothesis for this specific aim.

\section{$\underline{\text { Findings for Mothers and Child Sleep Variables }}$}

The comparison of mother and child sleep variables (sleep efficiency, actual sleep time, sleep duration, nocturnal awakenings and WASO) are demonstrated in Table Q-5. Results from the longitudinal mixed effect model using PROC Mixed are presented in Table Q-6. Mother sleep variables were used as the dependent variables in the analysis and child sleep variables and week were used as covariates. For this analysis, week 2 was coded as baseline. Mothers had a significantly lower sleep time (actual sleep time and sleep duration) compared to the child's sleep time. Sleep duration for the mothers and their children was significantly higher when on dexamethasone compared to when off dexamethasone.

\section{Findings for Fathers and Child Sleep Variables}

The comparisons of father and child sleep variables are shown in Table R-4. Sleep efficiency scores for fathers were less than their child during week 1 (59.60 and 71.63 respectively). Fathers had better sleep efficiency scores during week 2 (81.71) while their child's sleep efficiency score (65.67) decreased during week 2. Fathers had less actual sleep time recorded by actigraph (265.77 minutes) in week 1 compared to their ill child (343.95 minutes). But actual sleep time in week 2 for fathers was higher than their child's actual sleep time. Sleep duration scores for fathers were higher than their child's sleep duration scores in both week 1 and week 2 (Table R-4). There was a noticeable difference between number of nocturnal awakenings for father and child dyad in week 2 (during dexamethasone) as compared to week 1 . The average number of nocturnal awakenings for fathers was 7.56 while the average number of awakenings for the children was 16.77 . 
Table 4-10: Summary of Proc Mixed Model Regression Findings for Matched Primary Caregiver and Child.

\begin{tabular}{llll}
\hline $\begin{array}{l}\text { Primary Caregiver } \\
\text { Response Variable }\end{array}$ & Covariate & Coefficient & P-value \\
\hline Sleep Efficiency & Independent Variable & 0.04 & 0.69 \\
& Child Sleep Efficiency & -0.70 & 0.78 \\
Actual Sleep Time & Week & 0.31 & $0.002^{*}$ \\
& Child Actual Sleep Time & -46.3 & 0.07 \\
Sleep Duration & Week & 0.34 & $0.001 *$ \\
& Child Sleep Duration & -69.9 & $0.007 *$ \\
Nocturnal Awakenings & Week & 0.13 & 0.17 \\
& Child Nocturnal Awakenings & -0.86 & 0.50 \\
WASO & Week & -0.003 & 1.0 \\
\hline$* p=<0.05$ & Child WASO & -14.4 & 0.31 \\
\hline
\end{tabular}




\section{Findings for Couples and Child Sleep Variables}

Table S-2 presents the comparison of matched mother, father and child sleep actigraph variables descriptively. The children had consistently higher average efficiency scores for week 1 and week 2 than their parents. Mothers had higher sleep efficiency scores than the fathers. Actual sleep time for the children remained consistent between week 1 and week 2 . However their parents' (mothers and fathers) actual sleep time increased during week 2 (on dexamethasone) compared to week 1. During week 1 fathers' average sleep duration (519 minutes) was longer than that of their spouses and their ill child (490.51 minutes and 505.36 minutes respectively). During week 2, the mothers' average sleep duration (567.45 minutes) was longer than their spouses (505.70 minutes) and of their ill child (543.93 minutes). Interestingly, the numbers of nocturnal awakenings for the triad were in close proximity. Fathers had the highest number of nocturnal awakenings (13.60) which was recorded during week 1.

\section{Specific Aim Three - Parent Fatigue}

Compare parent fatigue as measured by parent self-report during the two consecutive 5-day periods of their child being off (week 1) versus on (week 2) dexamethasone.

Related hypothesis three: Parent fatigue scores will be less before dexamethasone (week 1) than during dexamethasone (week 2).

\section{Findings for Brief Fatigue Inventory Results for Primary Caregivers}

The primary caregivers' BFI measurements (parent fatigue) were assessed on day 2 and 5 when their children were off and on dexamethasone. Descriptive statistics for Primary Caregivers Fatigue scores are shown in Table 4-11. Overall the primary caregivers reported low fatigue scores. Scores ranged from 1 to 10 with 10 indicating a high level of fatigue. The average BFI scores when the children were on dexamethasone (week 2) were higher compared to when they were off dexamethasone (week 1). There was a significant difference in the average parent fatigue scores on week 1 day 5 compared to week 2 day $5(p=0.03)$. There was a trend of higher parent fatigue scores during week 1 compared to week $2(p=0.06)$. However, there was no significant different in parent fatigue during the two consecutive 5-day periods using PROC Mixed analysis $(p=0.23)$. These results support the hypothesis for this specific aim.

\section{Findings for Brief Fatigue Inventory Results for Mothers}

Descriptive statistics for fatigue scores of mothers are shown in Table Q-7. The average reported BFI scores by mothers in week 2 were higher than those in week 1. There was a trend of higher mother fatigue scores during week $1(p=0.07)$ Table Q-8. 
Table 4-11: Descriptive Brief Fatigue Inventory Results for Primary Caregivers.

\begin{tabular}{lllllll}
\hline Week/Day & $\mathrm{n}$ & Mean & $\mathrm{SD}$ & $\mathrm{Mdn}$ & $\mathrm{Min}$ & $\mathrm{Max}$ \\
\hline Week 1 Day 2 & 17 & 2.99 & 1.68 & 3.33 & 0.44 & 5.56 \\
Week 1 Day 5 & 17 & 2.61 & 1.66 & 2.67 & 0.00 & 6.89 \\
Week 2 Day 2 & 15 & 3.14 & 1.42 & 3.00 & 1.00 & 5.11 \\
Week 2 Day 5 & 16 & 3.44 & 1.78 & 3.17 & 1.00 & 7.67 \\
\hline
\end{tabular}


Using PROC mixed analyses, there was no significant difference in mother fatigue scores during two consecutive 5-day periods of off versus on dexamethasone (week1 versus week 2) $(p=0.19)$.

\section{Findings for Brief Fatigue Inventory Results for Fathers}

Fatigue scores for the fathers were also low. This is consistent with fatigue scores for primary caregivers and mothers in this study. The descriptive statistics for the fathers' BFI reports for week 1 and week 2 are shown in Table R-5. Fatigue scores for the fathers in this current study were highest during day 2 of week 1 (4.35). Fathers reported lower scores on day 5 of week 1 and week 2 (2.91 and 2.98 respectively).

\section{Findings for Brief Fatigue Inventory Results for Couples}

Both mothers and fathers reported low fatigue scores. However, fathers reported higher fatigue scores during both time periods than mothers except on day 5 of week 2 (during dexamethasone). The average BFI score for mothers on week 2 day 5 was 3.87 . Fathers reported the highest score on the BFI on day 5 of week 1 (3.89). The descriptive statistics for the couples' BFI scores for week 1 and week 2 are shown in Table S-3.

\section{Specific Aim Four - Parent Caregiver Burden}

Compare parental caregiver burden (time and effort related to care responsibilities) during the two consecutive 5-day periods as measured by the Revised Informal Care Survey and Care of My Child with Cancer Instrument.

Related hypothesis four: Parental subjective and objective burden scores will be greater during dexamethasone (week 2) than before dexamethasone (week 1).

\section{Findings for Primary Caregivers}

\section{Revised Informal Caregiver Survey Results for Primary Caregivers}

The majority of the primary caregivers completed the RICS so there was minimal missing data for this survey. The descriptive statistics for the Revised Informal Caregiver Survey (RICS) for week 1 and week 2 are shown in Table 4-12. Generally, the primary caregivers had low levels of subjective burden given that the maximum possible score is 51 . Week 2 RICS scores were significantly higher compared to week $1(p=0.05)$. Higher mean RICS scores for primary caregivers were noted for the following items: their child being upset, limits on their free time, limits on their social life, giving constant attention to their ill child, caregiving when they don't feel well, emotional strain and 
Table 4-12: Descriptive Revised Informal Caregiver Survey Results for Primary Caregivers $(n=16)$.

\begin{tabular}{lcccccc}
\hline Week & Mean & SD & Mdn & Min & Max & $p$ \\
\hline Week 1 RICS Score & 12.69 & 6.01 & 11.00 & 1.00 & 21.00 & \\
Week 2 RICS Score & 14.81 & 7.32 & 13.50 & 4.00 & 27.00 & \\
& & & & & & \\
Difference of Average & 2.13 & 4.01 & 2.00 & -5.00 & 11.00 & 0.05 \\
RICS Scores for Week 1 & & & & & & \\
and Week 2 & & & & & & \\
\hline
\end{tabular}
$* p=<0.05$ 
doing tasks that their ill child could be doing themselves. Each of the listed items had higher scores in week 2 (during dexamethasone) compared to week 1 (before dexamethasone) with the exception of the item that addressed giving their ill child constant attention. This particular item remained constant in week 1 and week 2 (1.00). The primary caregivers had the highest score during week 2 day 5 on the item that addressed social life (1.40).

\section{The Care of My Child with Cancer Instrument Results for Primary Caregivers}

Descriptive statistics for the CMCC scores are shown in Table 4-13. The emotional care, family relationship, communication and education domains had notably higher demand scores in week 2 than week 1. Scores for each item in the domains range from 1 to 5; with 5 representing a higher demand score. Moderate demand scores range from 2.5 to 3.4. The highest possible total score for the physical care domain is 65 (range 1 to 65 ). Items in the physical care domain that had higher demand means for both time periods (week 1 and week 2) for primary caregivers were item numbers 12 (Travel for medical care) and 13 (Time spent for appointments) (Appendix A) with item number13 having one of the higher item mean values during week 2 (3.43).

The highest possible demand score for the emotional care domain is 25 (range 1 to 25). Item numbers 14 (Providing support to child), 17 (Providing emotional support), 18 (meeting own support needs) (during week 2), and 19 (during week 2) of the emotional care domain had the highest demand means for the primary caregivers (Appendix A). The mean score for the finance item for week 1 and week 2 for primary caregivers were 1.49 and 1.54 respectively (with 5 being the highest score for this domain). The highest possible total score for the family/interpersonal relationships domain is 20. Primary caregivers had highest means on item numbers 22 (Planning activities for child), 23 (Planning activities for the family) and 24 (Getting babysitting). (Appendix A) Item number 23 of this domain had a mean demand score of 3.43 in week 2 .

There are only 2 items in the communication domain. The highest possible total demand score for this domain is 10 . The mean demand scores for item number 26 (Communicating to community about child's illness) for week 1 and week 2 were 1.36 and 1.48 respectively. The mean demand scores for item number 27 (Watching and reporting child symptoms) for the two time periods were 1.67 and 1.78 respectively. The mean scores for the item in the education domain were 1.73 and 2.08 for week 1 and week 2. Consistent with the RICS scores, the demand scores for the CMCC were relatively low.

The differences in average CMCC scores for week 1 and week 2 are shown in Table 4-14. The CMCC scores of physical care, emotional care, finances, family relationship, communication and education domains were not significantly different during the two consecutive 5-day periods of off versus on dexamethasone (week 1 versus week 2). The total score summing all domains was not calculated due to missing data. 
Table 4-13: Descriptive Care of My Child with Cancer Instrument Results for Primary Caregivers $(\mathrm{n}=14)$.

\begin{tabular}{|c|c|c|c|c|c|c|}
\hline Domain & Week & Mean & SD & Mdn & Min & Max \\
\hline \multirow[t]{2}{*}{ Physical Care (13 items) } & 1 & 18.76 & 7.29 & 17.11 & 9.00 & 40.08 \\
\hline & 2 & 18.44 & 6.28 & 17.40 & 10.00 & 34.93 \\
\hline \multirow[t]{2}{*}{ Emotional Care (7 Items) } & 1 & 12.81 & 4.00 & 12.51 & 3.00 & 22.81 \\
\hline & 2 & 13.85 & 6.15 & 14.19 & 1.00 & 29.91 \\
\hline \multirow[t]{2}{*}{ Finances ( 1 item) } & 1 & 1.49 & 0.48 & 1.41 & 1.00 & 2.00 \\
\hline & 2 & 1.54 & 0.47 & 1.41 & 1.00 & 2.45 \\
\hline \multirow[t]{2}{*}{ Family Relationships (4 items) } & 1 & 9.73 & 2.50 & 9.73 & 6.66 & 15.22 \\
\hline & 2 & 10.39 & 3.77 & 10.25 & 1.00 & 17.46 \\
\hline \multirow[t]{2}{*}{ Communication (2 items) } & 1 & 2.91 & 1.00 & 2.91 & 1.73 & 5.46 \\
\hline & 2 & 3.12 & 1.30 & 3.00 & 1.73 & 5.91 \\
\hline \multirow[t]{2}{*}{ Education (1 item) } & 1 & 1.73 & 0.93 & 1.41 & 1.00 & 4.00 \\
\hline & 2 & 2.08 & 1.25 & 2.00 & 1.00 & 5.00 \\
\hline
\end{tabular}


Table 4-14: Differences in Care of My Child with Cancer Instrument Results for Primary Caregivers.

\begin{tabular}{llllllll}
\hline $\begin{array}{l}\text { Difference in Average } \\
\text { (Week 2-Week 1) }\end{array}$ & Mean & SD & Mdn & Min & Max & $t$ & $p$ \\
\hline Physical Care (13 items) & 1.73 & 6.47 & 0.45 & -9.54 & 18.52 & 1.00 & 0.33 \\
Emotional Care (7 Items) & 0.48 & 6.20 & 0.00 & -11.11 & 17.40 & 0.29 & 0.78 \\
Finances (1 item) & 0.13 & 0.46 & 0.00 & -0.59 & 1.45 & 1.01 & 0.33 \\
$\begin{array}{l}\text { Family Relationships } \\
\text { (4 items) }\end{array}$ & 1.41 & 3.25 & 1.16 & -2.60 & 10.58 & 1.57 & 0.14 \\
Communication (2 items) & 0.21 & 1.48 & 0.00 & -1.46 & 3.91 & 0.52 & 0.61 \\
Education (1 item) & 0.30 & 0.83 & 0.00 & -0.54 & 2.55 & 1.30 & 0.22 \\
\hline$* p=<0.05$ & & & & & & &
\end{tabular}


Total demand scores for the CMCC could not be calculated if responses were missing for either time or effort for any item. The data were examined for pattern of missing responses. There was no trend of missing responses for either time or effort nor was there any one demand item that had fewer responses. The pattern of missing responses for this current study instrument was random. The comparison between CMCC demand scores and RICS scores was not done because the total demand scores for CMCC could not be calculated for primary caregivers. These results support the hypothesis for this specific aim.

Findings for Mothers

\section{Revised Informal Caregiver Survey Results for Mothers}

The descriptive statistics for the Revised Informal Caregiver Survey (RICS) for week 1 and week 2 are shown in Table Q-11. Week 2 RICS scores were significantly higher compared to week $1(p=0.05)$. The items that addressed "taking care of their ill child when they did not feel well" and "taking care of ill child being hard emotionally" were the only 2 items that the mothers scored lower in week 2 compared to week 1 .

\section{Care of My Child with Cancer Instrument Results for Mothers}

Descriptive statistics for CMCC scores are shown in Table Q-11. The differences in average demand scores of physical care, emotional care, finances, family relationship, communication and education domains were not significantly different during the two consecutive 5-day periods of off versus on dexamethasone (week 1 versus week 2) (Table Q-12). These findings imply that time and effort related to parental caregiving did not differ between the two time periods.

\section{Findings for Fathers}

\section{$\underline{\text { Revised Informal Caregiver Survey Results for Fathers }}$}

Fathers reported low scores for subjective caregiver burden. The descriptive statistics for the revised Informal Caregiver Survey (RICS) for week 1 and week 2 are shown in Table R-6. Fathers had notably higher scores on numbers 4 (child becomes upset), 8 (limits free time), 9 (limits social life), 11 (constant attention), 13 (care taking when not feeling well) and 14 (care hard emotionally) of the RICS (Appendix B). The highest mean RICS scores for fathers were during week 2 for item numbers 4 and 9 (Appendix B). 


\section{Care of My Child with Cancer Instrument Results for Fathers}

There was minimum difference noted in the average of CMCC demand scores for week 1 and week 2 for the fathers. The highest average demand scores were reported for physical and emotional care domains, which is consistent with CMCC results of primary caregivers. The descriptive statistics for the revised CMCC for week 1 and week 2 are shown in Table R-7.

\section{Findings for Couples}

\section{Revised Informal Caregiver Survey Results for Couples}

Both mothers and fathers reported higher subjective burden scores as measured by the RICS in week 2, (when their child was on dexamethasone), than in week 1 (when their child was off dexamethasone). These results are consistent with the findings for the primary caregiver RICS results. The descriptive statistics for the RICS results for couples during week 1 and week 2 are shown in Table S-4. The RICS scores for mothers were higher in week 2 than were the fathers' scores in week 2 . However, the fathers had the higher scores in week 1. Similar mean scores were noted for fathers and mothers on item number 6 (My child's behavior embarrasses me) for week 1 and week $2(0.57,0.67)$ and $(0.57,0.71)$ respectively. Both fathers and mothers had low scores for item number 12 ("Taking care of my child has caused my health to get worse"). Fathers had a mean score of 0.00 and 0.20 for week 1 and week 2, while mothers had a mean score of .05 for both week 1 and week 2 . Mothers had higher scores ( 0.93 and 1.00 respectively) for both time periods on item number 17 (My sleep is interrupted because I have to care for my child) than fathers for this same item $(0.50$ and 0.40$)$.

\section{Care of My Child with Cancer Instrument Results for Couples}

The descriptive statistics for CMCC results for couples during week 1 and week 2 are shown in Table S-5. Fathers reported greater CMCC demand scores in the emotional support domain, while mothers reported higher physical care demand scores. Total CMCC scores were greater for fathers than mothers in both week 1 and week 2 .

\section{Specific Aim Five - Relationships among Sleep, Fatigue, and Caregiver Burden}

Assess the relationships among parent sleep quality, fatigue, and caregiver burden within each of the 5-day periods.

Related hypothesis five: Parental sleep would be inversely related to parent fatigue and parent caregiver burden. Average parental scores for sleep quality, fatigue, 
and caregiver burden before dexamethasone (week 1) will be less than average scores during dexamethasone (week 2).

The PROC MIXED model technique was used to assess the relationships among parent sleep variables, fatigue and caregiver burden during the on and off dexamethasone study period. Fatigue measurements were used as dependent variables and sleep quality variables and caregiver burden (RICS scores) were used as covariates in the model. The model was run independently for each of the sleep variables (efficiency score, actual sleep time, sleep duration, nocturnal awakenings and WASO). Week 2 was coded as baseline in the model.

In each independent model, fatigue scores were significantly associated with caregiver burden $(p=<0.001)$ adjusting for the sleep variable and exposure to dexamethasone (week). Fatigue was not statistically associated with sleep or exposure to dexamethasone. These results partially support the hypothesis for this specific aim. These results should be interpreted with caution because of the small number of participants used in the model (Table 4-15).

\section{Specific Aim Six - Parent Sleep, Fatigue, Caregiver Burden, and Demographic Variables}

Explore if parent sleep quality, fatigue and caregiver burden differs by parent age, marital status, ethnicity, child's risk category (low versus standard) or the child's age.

The PROC MIXED model technique was used to explore if parent sleep quality, fatigue and caregiver burden differs by parent age, marital status, ethnicity, child's risk category (low versus standard), or the child's age. These results should be interpreted with caution due to the small sample status used in the univariate model. Each dependent variable (sleep quality (efficiency score, actual sleep time, sleep duration, nocturnal awakenings, and WASO), fatigue or caregiver burden) was regressed on each independent variable (Parent's age, marital status, ethnicity, child's risk category and child's age). Parent's age and child's age were entered as continuous variables in the model while marital status (married or divorced or single), ethnicity (white or others), child's risk category (low or standard) were entered as categorical variables. Table 4-16 displays the values of coefficient from each model.

Sleep duration was significantly associated with child's risk category (coefficient $=90.5$ when standard risk was coded as baseline, $p=0.01$ ). Since standard risk is coded baseline $(=0)$, then a coefficient that is positive $(90.5)$ means that the parents of low risk ALL patients sleep duration was 90.5 minutes more compared to parents of children with standard risk ALL. The other sleep quality indicators (efficiency score, actual sleep time, sleep duration, nocturnal awakenings, and WASO) were not statistically significant associated with parent's age, ethnicity, marital status, child's risk category or child's age. Fatigue (BFI score) was not significantly associated with parent's age, ethnicity, marital status, child's risk category or child's age. Subjective 
Table 4-15: Summary of Proc Mixed Model Regression Findings Related to Sleep, Fatigue, and Caregiver Burden within each of the 5-day Periods.

\begin{tabular}{|c|c|c|c|}
\hline Response Variable & Covariate & Coefficient & $p$-value \\
\hline \multirow[t]{3}{*}{ Fatigue } & Week & 0.14 & 0.31 \\
\hline & Efficiency Score & -0.002 & 0.66 \\
\hline & Subjective Caregiver Burden & 0.29 & $<0.001 *$ \\
\hline \multirow[t]{3}{*}{ Fatigue } & Week & 0.14 & 0.33 \\
\hline & Actual Sleep Time & -0.00006 & 0.91 \\
\hline & Subjective Caregiver Burden & 0.29 & $<0.001 *$ \\
\hline \multirow[t]{3}{*}{ Fatigue } & Week & 0.19 & 0.19 \\
\hline & Sleep Duration & 0.0006 & 0.247 \\
\hline & Subjective Caregiver Burden & 0.29 & $<0.001 *$ \\
\hline \multirow[t]{3}{*}{ Fatigue } & Week & 0.14 & 0.33 \\
\hline & Nocturnal Awakenings & 0.014 & 0.16 \\
\hline & Subjective Caregiver Burden & 0.29 & $<0.001^{*}$ \\
\hline \multirow[t]{3}{*}{ Fatigue } & Week & 0.15 & 0.29 \\
\hline & WASO & 0.0005 & 0.59 \\
\hline & Subjective Caregiver Burden & 0.29 & $<0.001 *$ \\
\hline
\end{tabular}


Table 4-16: Results for Univariate Regression Analysis Assessing Relationship between Sleep Quality, Fatigue, Caregiver Burden, Parent's Age, Ethnicity, Marital Status, Child's Risk Category, and Child's Age.

\begin{tabular}{llllll} 
& \multicolumn{5}{c}{ Independent Variables } \\
\cline { 2 - 6 } Dependent Variables & $\begin{array}{l}\text { Parent's } \\
\text { Age }\end{array}$ & Ethnicity & $\begin{array}{l}\text { Marital } \\
\text { Status }\end{array}$ & $\begin{array}{l}\text { Child's Risk } \\
\text { Category }\end{array}$ & $\begin{array}{l}\text { Child's } \\
\text { Age }\end{array}$ \\
\hline Sleep Efficiency & 0.84 & 0.88 & 4.4 & -7.7 & 1.57 \\
Actual Sleep Time & 5.9 & 23.0 & 43.0 & 12.7 & 2.8 \\
Sleep Duration & -0.93 & 10.0 & -12.2 & $90.5^{*}$ & -10.3 \\
Nocturnal Awakenings & 0.19 & -7.2 & 1.3 & -1.0 & -0.06 \\
WASO & -3.5 & -5.3 & -30.3 & 50.6 & -7.9 \\
Fatigue (BFI Score) & 0.05 & -1.0 & 0.44 & 0.001 & 0.08 \\
$\begin{array}{l}\text { Caregiver Burden (RICS } \\
\text { Score) }\end{array}$ & -0.25 & 2.57 & -1.80 & 2.19 & -0.004 \\
\hline
\end{tabular}

Note: Results represent values of coefficients from each model $* p=0.01$ 
caregiver burden (RICS score) was not significantly associated with parent's age, ethnicity, marital status, child's risk category or child's age. Objective caregiver burden was not analyzed in this model because total CMCC scores were not available for primary caregivers.

\section{Summary}

In summary, primary caregivers had significantly higher actual sleep time and sleep duration, measured by actigraphy, when their child was on dexamethasone compared to when their child was off dexamethasone. These results do not support the proposed hypothesis related to aim one. Sleep duration recorded by sleep diary report was not statistically different between week 2 and week 1 . However, sleep duration reported by diary report for week 1 day 2 was significantly higher compared to those recorded by actigraph $(p=0.025)$.

Hypothesis two states that the average sleep quality scores before dexamethasone would be greater than the average sleep quality scores during dexamethasone and that these parent and child sleep scores will have a directional relationship to one another. Fourteen parent/child dyads were examined for this related hypothesis. Primary caregivers had a significantly lower actual sleep time and sleep duration compared to their child's actual sleep time and sleep duration during week 1 and week 2 . However, both primary caregivers and their children slept longer during week 2 (during dexamethasone). The number of nocturnal awakenings was also similar for the parent/child dyad. There were significantly positive associations between primary caregiver and child actual sleep time and sleep duration. These results partially support hypothesis two.

Overall, primary caregivers reported low fatigue scores. However, higher fatigue scores were reported when the children were on dexamethasone as compared to when the children were off dexamethasone. This finding supports the hypothesis related to Aim three; caregiver fatigue scores will be less before dexamethasone than caregiver fatigue scores during dexamethasone.

Subjective burden scores for parents were significantly higher in week 2 (on dexamethasone) than in week 1 (off dexamethasone $(p=0.05)$. Average scores for objective burden were also slightly higher during week 2 as compared to week 1 . These results support hypothesis four (parental subjective and objective burden scores will be greater during dexamethasone (week 2) than before dexamethasone (week 1).

Proc Mixed model technique was used to assess the relationships among primary caregiver sleep variables, fatigue and caregiver burden during the on and off dexamethasone study period. Fatigue scores were significantly associated with subjective caregiver burden but were not statistically associated with sleep or exposure to dexamethasone. These results partially supported hypothesis five. 
The Proc Mixed model technique was used to explore if parent sleep quality, fatigue and caregiver burden differs by parent age, marital status, ethnicity child's risk category (low versus standard), or the child's age. Sleep duration was significantly associated with child's risk category (coefficient $=90.5$ when standard risk was coded as baseline, $\mathrm{p}=0.01$ ). There were no other statistically significant associations found among fatigue or caregiver burden by parent age, marital status or ethnicity. These results should be interpreted with caution due to the small sample size. 


\section{CHAPTER 5: DISCUSSION AND IMPLICATIONS}

\section{Discussion}

This study is a systematic examination of caregiver burden, fatigue and sleep quality in parents of children undergoing steroid therapy during continuation treatment for ALL. These variables were described within two time periods, the first when their child was not taking dexamethasone (week 1) and the second (week 2), when their child is taking dexamethasone. Additionally, this study compared parent's sleep quality data with that of their child's data during the time periods as described above. This chapter includes an overview and interpretation of findings for each specific aim. Strengths and limitations of the study, along with implications for policy, practice, theory and future research are also addressed. While the findings of this study are preliminary, they may provide direction for future studies that examine parent sleep, fatigue and caregiver burden in larger samples and insight for potential interventions that may address the needs of parent caregivers of children receiving steroid therapy in a similar phase of treatment for ALL.

\section{$\underline{\text { Parent Caregiver Characteristics }}$}

Initially it was projected that 39 female guardians would be enrolled and fully evauable for study aims. Thirty-five eligible parental caregivers were approached to be on this study. Of the eligible caregivers, twenty-four participants consented to participate in the study and 22 completed study requirements (fifteen mothers and seven fathers). Seventeen parents were identified as primary caregivers. Thirteen parents declined to participate for an overall rate of refusal of $31.4 \%$. Reasons for refusal varied. Parents $(n=4)$ stated that they were too busy to participate. One mother lost her home in hurricane Katrina and was unable to consider the additional responsibilities associated with the study participation. Parents in this current study also completed study forms assessing their child's sleep and fatigue in addition to study forms for the parent study. According to Gattuso et al. (2006), child and parent refusal rates when asked to participate in clinical research studies have ranged from of $6.7 \%$ to $46.7 \%$. Refusal rates varied depending on the study design and topic. Studies with two or more data collection times, such as this current study, had refusal rates that ranged from $6.7 \%$ to $38.5 \%$. The refusal rate for this study may indicate a need for change in study design. Refusal rates for this study were closer to the higher end of the range. This could explain why some relationships and or characteristics that may truly exist in this current study population were not identified.

Eighty-eight percent of the primary parent caregivers in this study were females $(15 / 17)$. This is consistent with the literature as mothers are most identified as primary caregivers (Sheeran et al., 1997; Walsh et al., 2004; \& Svavarsdottir, 2005b). The ages of the primary caregivers ranged from 28 years to 47 years with mean age of 38 years. Seventy-one percent (12/17) of the primary caregivers were married and forty-seven 
percent had 3 or more dependents. Research studies about parent caregivers of children with cancer reported mean ages at approximately 36.9 years for mothers and 37.9 years for fathers with a range between 25 to 48 years, with most parents being married with and having an average of 2 children (Yeh, 2002; Svavarsdottir, 2005b; Lahteenmaki et al., 2004). Most of the caregivers in this study were White. This characteristic reflects the patient population that is most affected by ALL (Pui, 2006). Participants in this study are white mothers between the ages of 28 and 47 that are married and have some college education. Thus it is inappropriate to generalize the study's results to all caregivers of children with cancer. However, because this study primarily focuses on mothers who are most often the primary caregivers, these findings would be useful in future studies about mothers of the same ethnic group. Few fathers participated in this study and therefore it would be inappropriate to generalize findings to male caregivers.

\section{$\underline{\text { Aim One - Parent Sleep }}$}

The first research aim focused on describing sleep quality in parent caregivers during week 1 (when their child was off dexamethasone) and week 2 (when their child was on dexamethasone) using sleep diary and actigraph measures. Several studies document adult actual sleep time, sleep efficiency and night time awakenings using actigraph and sleep diary measures (Mindell \& Durand, 1992; Jean-Louis et al., 1999; Jean-Louis et al., 2000; Lauderdale et al., 2006). Sleep efficiency scores for the parents in this current study were $73.47 \%$ in week 1 and $75.66 \%$ in week 2 . Ideal sleep efficiency scores for healthy adults is $85 \%$ or higher per night (DSM-IV-TR, 2000). Studies about gender differences in sleep in healthy adults report a mean efficiency score by actigraph for women at $92 \%$ and $88 \%$ for men (Jean-Louis et al., 1999). The percentage of time spent in bed asleep (sleep efficiency) changed minimally (approximately $2 \%$ ) for primary caregivers between the two time periods. This small change may indicate that the caregivers sleep efficiency is not affected by their child being on dexamethasone because they already have poor sleep efficiency. There is almost a 19\% difference in the efficiency scores of the primary caregivers in this study and that of healthy adults. This is an important finding consistent with published literature related to sleep problems in caregivers.

The mean actual sleep time recorded by actigraph for primary caregivers (men and women) in this current study was 330.79 minutes in week 1 (before dexamethasone) and 391.04 minutes in week 2 (during dexamethasone). Primary caregivers self-report approximately an hour more sleep during week 2 . These sleep times are lower than those reported by healthy adults and lower than sleep times of parents with children that have sleep disorders. Normal actual sleep time (total minutes of night time sleep) for adults is 6 to 8 hours per night (DSM-IV-TR, 2000). Night time sleep for fathers and mothers of children with sleep disorders are reported as 460 minutes and 471 minutes respectively (Mindell \& Durand, 1992). These results indicate that the parental caregivers in this current study not only have poorer sleep quality compared to healthy adults but they also have poorer sleep quality when compared to other parents of children with sleep 
disorders. This demonstrates a relationship between poor sleep quality in parents and caring or children diagnosed with illnesses associated with sleep disruptions.

In this current study, primary caregivers' average sleep duration measured by sleep diary was 560 and 507 minutes during week 1 and 497 and 390 minutes during week 2 . Sleep duration measured by actigraph for primary caregivers was 468 minutes and 409 minutes in week 1 and 509 and 486 in week 2. The standard deviation for sleep duration by actigraph more than doubled from 53.79 in week 1 to 122.01 in week 2 . This suggests increase in variability in sleep duration during week 2 . The maximum minutes of sleep for week 2 was 867 minutes (14 hours) and the minimum was 422 minutes (7 hours). This finding speaks to the variability that exists when examining a variable like sleep duration. This result included sleep duration results from both male and female participants. As documented previously, there are gender differences in sleep and these differences may have contributed to the variability in sleep duration.

Notable differences in parent caregiver's sleep duration by actigraph and selfreport were found in this study. During week one, primary caregivers had less sleep duration as measured by actigraphy than indicated in the sleep diary self-report. This is consistent with literature reporting that the mean sleep duration for healthy men and women by actigraph was 373 minutes and mean sleep duration of 415 minutes by selfreport (Girardin et al., 1999; Girardin et al., 2000). Sleep duration measured by selfreport seems to be greater than sleep duration measured by actigraph. According to Ohayon et al. (2004), the average sleep duration by self-report for adults aged 35 to 50 years ranges from 5.4 (324 minutes) to 7.8 (468 minutes) hours. Actigraph data for week 1 report that the primary caregivers were getting less sleep during week 1 compared to sleep diary reports for week 1 but the opposite results by actigraph were reported in week 2 . Primary caregivers perceived that they were getting less sleep during week 2 . There was a significant difference in sleep diary results and actigraph results for sleep duration during week 1 day 2 for primary caregivers $(p=0.025)$. This result could be due to biased reporting, since the parents knew their child was on dexamethasone.

WASO scores during week 1 and week 2 for primary caregivers in this study were 106.08 and 124.54 minutes respectively and nocturnal awakenings for primary caregivers were 14.05 for week 1 and 14.33 for week 2 . There was little change in nocturnal awakenings for primary caregivers between the two time periods (mean difference 0.28 and standard deviation of 5.10). Adults usually awaken 1-2 times per night (Mindell \& Durand, 1992), and have a WASO of 30 minutes or less per night (DSM-IV-TR, 2000). Although actigraph data for primary caregivers indicated increased sleep duration during week 2 , these caregivers had numerous nocturnal awakenings and sometimes remained awake up to 2 hours. Again, these results further emphasize that these parents indeed have poor sleep quality.

Mothers in this current study had higher sleep duration and actual sleep time than the fathers in this study. Data for this current study are consistent with the literature. In a study examining sleep in parents of children with leukemia, researchers observed that 
sleep duration captured by actigraphy was higher in mothers than in fathers (Gedaly-Duff et al., 2006). Mothers slept 6 to 7.8 hours while the fathers slept 4.5 to 7.8 hours.

Both the fathers and mothers in this current study reported higher sleep duration time (on sleep diary) than what was recorded by actigraphy. Although parents in this study selfreported higher sleep duration than the participants in the literature, the trends are congruent.

Clearly, the results of this study reveal that the parents in this study have poor sleep quality. Their sleep duration, WASO, nocturnal awakenings and sleep efficiency scores are different from the norms for healthy adults. It was hypothesized that sleep quality would be less during week 2 (during dexamethasone) than in week 1 (before dexamethasone). Surprisingly, the results were just the opposite. Parent caregivers had higher sleep duration and actual sleep time when their child was on dexamethasone. The results were the same for actigraph and sleep diary data. The WASO results for fathers were the only sleep quality indicator that was lower during week 2. Parent caregivers of this sleep study perceived that they were getting less sleep during the time their child was on dexamethasone and reported less sleep during week 2 than actigraphy results for week 2. Possibly, parents anticipated that their sleep would be less because their child was on dexamethasone. The National Sleep Foundation (2004) reported that parents who perceive that their children have a sleep problem are more likely to report getting too little sleep themselves.

There were some notable findings from the sleep diary reports. The primary caregivers in this study rarely reported taking naps. They also reported that exercise activity decreased in week 2 . More than half of the primary caregivers rated their sleep as average or refreshed on day 5 of week 2. Caregivers also reported more sleep interruption by their child in during week 2 . No caregivers reported that their sleep was refreshed during week 2 day 5. Rarely did primary caregivers report sound sleep. Participation in completing the sleep diary declined by $50 \%$ on day 5 of week 2 . Parents may have gotten overwhelmed with caring for their child on dexamethasone or burdened by study requirements as the parents also had study materials to complete for their child on the SLEEP study.

These sleep diary reports may suggest that even though the parents spent longer time in bed during week 2 , although their sleep may not have been quality sleep. This may be due to the extended disruptions in sleep. Prolonged disruptions in sleep can contribute to disruptions in normal cycle. A full sleep cycle consists of a balance of Nonrapid eye movement sleep (NREM) and Rapid Eye Movement Sleep (REM) (Chokroverty, 1999). Poor quality sleep in these parents could also be attributed to psychological factors such as depression or anxiety.

\section{Aim Two- Parent and Child Sleep}

The second specific aim of this study focused on comparing sleep quality results between parent caregivers and their child with cancer. Primary caregivers' actigraph 
results for sleep efficiency, actual sleep time, and sleep duration in this current study were lower than that of their ill child. Gau and Merikangas (2004) reported similarities and differences among adults and their children with parents tending to have shorter night time sleep duration than their children. Likewise, a report by Meltzer and Mindell (2007) found that mothers had a total sleep time of 7.3 hours while their child had an average sleep time of 10 hours. Primary caregivers in this study slept an average 5.75 hours in week one and 6.26 hours in week 2, while the child slept an average of 6.29 hours in week one and 7 hours in week 2. These findings are below normal average sleep times for healthy adults and children and may indicate that parent caregivers and their cancer have poorer sleep quality than healthy children and adults who are not parents of a chronically ill child. This finding also supports the influence of an ill child's sleep pattern on their parent's sleep patterns and that caregivers of chronically ill family members experience poor sleep quality.

Both primary caregivers and their ill child had higher sleep efficiency, actual sleep time, and sleep duration during week 2 when the ill child was taking dexamethasone. While this was an unexpected result, parental and child sleep quality results did display a directional relationship. The parents' sleep increased as their child's sleep increased as their child's actual sleep time and duration increased. This direction of change remained the same for analysis of matched mother and child and matched father and child. The findings in this current sleep study are congruent to findings in some studies comparing parent and child sleep characteristics. Poor maternal sleep quality is related to more child sleep disruptions and prolonged sleep onset time in children (Meltzer \& Mindell, 2007). Gau et al. (2004) showed low correlations in sleep schedules and sleep-wake patterns in children and their parents who did not work shifts or night and evening hours. Compared to the children in this study, parents tended to have shorter nighttime sleep duration than their ill child. The number of nocturnal awakenings and WASO score for primary caregivers and their children were quite similar. There were also positive associations between primary caregiver and child sleep duration and actual sleep time. Sleep duration and actual sleep time for primary caregivers and their children were significantly higher when the child was on dexamethasone. Again, these findings suggest that the child's sleep influences parent sleep. But it also leads to the plausible explanation that may have been the parents and their children were more fatigued during the dexamethasone week and therefore spent more time in bed.

A study examining pain, sleep disturbance and fatigue in children with leukemia and their parents, found that children and parents reported symptoms related to sleep disturbances after outpatient chemotherapy. The children and parents (both mothers and fathers) in that study higher numbers of nighttime awakenings over a three-day period after outpatient chemotherapy as measured by actigraphy (Gedaly-Duff et al., 2006). The number of nocturnal awakening for the primary caregivers and children in this current study did not increase significantly during the dexamethasone week. It is likely that sleep disturbances are more noticeable after chemotherapy treatment as opposed to during the time of chemotherapy treatment. 
Fathers in this current study also had higher sleep efficiency scores, actual sleep time and sleep duration during week 2 (when their child is taking dexamethasone) than that of their ill child. Sleep quality indicators were higher during week 2 for the father and child dyad. The mother's sleep is documented to be more affected by her child's sleep than is the father's sleep (Meltzer \& Mindell, 2007). However, few studies have examined fathers' sleep quality in relation to their ill child's sleep.

\section{$\underline{\text { Aim Three- Parent Fatigue }}$}

This aim focused on parent fatigue scores during week 1 (when their child was not on dexamethasone) and week 2 (when their child was on dexamethasone). The Brief Fatigue Inventory (BFI) was used to measure parental fatigue in this current study. Primary caregivers generally reported low fatigue scores, with scores ranging from 2.99 to 3.44. The average reported BFI score when the children were on dexamethasone (week 2) were higher compared to when they were off dexamethasone (week 1). This finding, although not significant, was an expected outcome. This result may lend some explanation as to why parent caregivers had longer sleep duration during the week when their ill child was on dexamethasone. It is highly possible that these caregivers were fatigued and therefore spent more time in bed.

Fatigue scores were low for mothers, but were higher in week 2 than in week 1. However, fathers reported higher fatigue scores during both time periods than mothers. Although predictions were not made about fatigue outcomes between mother and fathers, this result also came as a surprise. Similarly, in a pilot study, by Gedaly-Duff et al. (2006) about pain, sleep disturbance, and fatigue in children with leukemia and their parents, fathers reported having an average fatigue score of 50 on a scale of 0 to 100 ( 0 meaning no fatigue) while the mothers in this same study reported an average fatigue score of 45 (on a scale of 0 to 100). The parents in this study reported low to moderate fatigue scores (means between 2.61 and 4.35 on a scale of 1 to 10). Fatigue score may have been low because parents under-reported their level of fatigue. It is also possible that during this point in treatment for their child's illness, parents have developed coping strategies and have adapted to their caregiving role and therefore do not report high levels of fatigue. It is possible that is socially undesirable to admit to being fatigue while caring for one's ill child.

\section{$\underline{\text { Aim Four- Parent Caregiver Burden }}$}

Parental Caregiver burden was assessed using the Revised Informal Caregiver Survey (a subjective measure) and the Care of My Child with Cancer Instrument (an objective measure) on day five of week 1(off dexamethasone) and on day five of week 2 (on dexamethasone). Primary caregivers in the current study reported low subjective burden scores for week 1 and week 2 with a mean values of 12.69 and 14.81 respectively on a scale of 0 to 51 ( 0 meaning no subjective burden). But the scores for subjective burden were higher during week 2 (when their child was on dexamethasone). This study 
takes place during the continuation phase of the child's treatment of cancer and the parents in this study may have adjusted to feelings of burden since child's diagnosis of cancer; therefore reporting low levels of burden. The RICS instrument is a newly revised measure for parents. Although the items in the RICS are reliable with standardized Cronbach alpha estimates ranging from 0.86 to 0.88 , these items may not have asked the appropriate questions to capture subjective burden in these parents. Another plausible reason may be that the questions invoked feelings of guilt and discomfort admitting to burden with caring for their ill child; thus under-reporting subjective burden scores.

Objective burden scores were generally low but average CMCC scores were higher during week 2 as well. These findings support the hypothesis that subjective and objective burden would be greater during week 2 (when the child was taking dexamethasone). There were some individual items in the CMCC that had moderate demand scores. The items with the highest demand scores for week 1 and week 2 included travel to and from the hospital (2.40 and 2.39), time spent at hospital for appointments (2.61 and 3.43), providing emotional support for the child (2.73 and 3.22), providing emotional support for spouse (2.51 and 2.30), planning activities for ill child around treatment and illness $(2.40$ and 2.39$)$, planning activities for family around treatment and illness (2.61 and 3.43), and getting childcare for ill child (2.73 and 3.22). Items with the higher demand score in the study by Keegan-Wells et al. (2002) were similar to the results in this current study. Higher demand scores were noted with items that included meeting emotional needs of sick child, attending medical appointments, comforting child through painful procedures, planning activities for the sick child, traveling for appointments, and disciplining the ill child. These tasks seem to be more time consuming and require more effort for parents with children cancer.

Mothers, in a longitudinal study of parents caring for a child with cancer (Svavarsdottir, 2005), reported the most time consuming caregiving task as providing emotional support for the child with cancer and other children in the family along with organizing and arranging services for the family. Providing emotional support for the family was also the most difficult caregiving task for mothers in this study. Fathers reported providing emotional support, working outside the home and organizing care for the ill child as the most tie consuming task while the most difficult task was providing emotion al support to the family and organizing care needs and managing working outside the home. Mothers were found to report higher caregiving activities than fathers (Svavarsdottir, 2005). The mothers in this current study reported higher demand scores in the physical care domain of the $\mathrm{CMCC}$, while fathers reported greater demand scores in the emotional support domain (Appendix A). Interestingly, contrary to the literature, fathers in this current study had higher total CMCC scores than mothers for both week 1 and week 2.

A plausible explanation for the relatively low burden scores may be that the parents have adapted to caring for their child with cancer by this point in treatment. This would support the reports by Svavarsdottir (2005) that caregiving demands decreased over time as the child's level of medical treatment becomes less intense. Boman et al. (2004) also support that parental distress depends on the illness type and time passed 
since diagnosis. Another explanation may be that parents feel as though they are complaining by addressing some of the issues related caregiver burden and therefore under-reported burden scores.

Aim Five - Relationships among Sleep, Fatigue, and Caregiver Burden

There was a significant relationship between fatigue scores and caregiver burden in this study. Both fatigue and caregiver burden increased during week 2 (when the child was taking dexamethasone). There was a positive relationship between the two variables. As fatigue increased, subjective burden increased. This finding is supported in the literature. The study by Jensen and Given (1991) found a positive relationship between the number of hours of daily care and severity of fatigue and the impact severity of fatigue on the caregiver's schedule. These researchers suggest that the more the caregiver's schedule is a burden, the greater will be the fatigue experienced.

It was expected that there would also be direct relationships among fatigue and sleep variables. However, fatigue was not statistically associated with sleep quality variables in this current study. This result may be due to the small sample size. There is an abundance of literature linking fatigue and sleep disturbance (Gay et al., 2004; Ohayon \& Shapiro, 2000; Lee, 2001). Increased fatigue may affect the quantity and quality of sleep wake-cycles and increased daytime sleepiness (Gedaly-Duff et al., 2006). Fatigue may also be underreported in these caregivers because of social desirability concerns. Under-reporting could contribute to the likelihood not finding a relationship between fatigue and sleep. Even though the literature repeatedly demonstrates a positive relationship between sleep and fatigue it is possible that no relationship exist between fatigue and sleep for these parents. This possibility could be explained if there are some psychological reasons for sleep disturbance and or fatigue which were not analyzed in this study. However, this study was not sufficiently powered to detect these associations.

\section{Aim Six - Parent Sleep, Fatigue, Caregiver Burden, and Demographic Variables}

In an exploratory manner, Proc Mixed model technique was used to see if parental sleep quality, fatigue and caregiver burned differs by parent age, marital status and ethnicity, child's risk category (low versus standard) or the child's age. Sleep duration was significantly associated with child's risk category (coefficient $=90.5$ when standard risk was coded as baseline, $p=0.01$ ). This was the only statistically significant association. This may mean that parents with children with standard-risk ALL may have different sleep duration than those parents of children with low-risk ALL. This may be related to differences in treatment regimen for risk groups. There may be increased anxiety and/or distress in parents who have a child diagnosed with standard risk ALL. In a study by Meltzer \& Mindell (2007), mothers of children who had significant sleep disruptions reported greater feelings of parenting stress and caregiving overload. 


\section{Strengths and Limitations}

The study has several limitations as well as strengths. Limitations of the study include small sample size, response rate, instrumentation, and design challenges. Strengths include the examination of a symptom cluster in a family context, examination of gender differences in caregiving, sleep and fatigue, and inclusion of both subjective and objective measures of study variables. Limitations are discussed first, followed by a discussion of study strengths.

This study has a small sample size and thus generalizability of findings is limited. Caregivers in this study were primarily female, white, married, homemakers and had some college. This sample is not truly representative of the overall population of families of children with ALL. Fathers and minorities were underrepresented in this current study.

This study included a convenience sample of 22 parent caregivers of children receiving treatment at St. Jude Children's Research Hospital in Memphis, Tennessee. Convenience sampling limits the external validity of a study (Burns \& Grove, 2001). In addition, many caregivers were not eligible to participate in this study because their child refused participation in the SLEEP study. Caregivers who were severely fatigued, burdened, and sleep deprived may not have been represented in this study sample. There was also a large amount of random missing data for the CMCC instrument. This instrument has 29 items including one open ended item. The parents in this study completed study instruments for themselves and their ill child in addition to wearing the actigraph for 10 days. Completing study instruments could have taken up too much time for the caregivers.

Instrumentation was also a study limitation. The study instruments used in this study were self-assessment tools (with the exception of the wrist actigraph). Surveys do not capture in-depth analysis of a topic but rather a broad overview. There are also some influences that are imposed by the questionnaires structure. The only information gained is what is asked important information can be overlooked (Talbot, 1995). Respondents can distort the results by giving misinformation. Self-report questionnaire inquire about the respondent's personal experiences. While some welcome the opportunity to express feelings and concerns regarding a particular topic other may not; especially if the questions are associated with socially unacceptable behaviors or attitudes (Polit \& Hungler, 1991). These study instruments may not have reflected the true experiences of the parent caregivers. It is possible that asking parents if caring for their child contributed to their fatigue, sleep and caregiver burden may have evoked negative or guilt-like feelings. Parents may feel uncomfortable or as though they were complaining about the care they have to give to their child with cancer (Caserta et al., 1996 \& Nijboer et al., 2001). Two instruments were used to capture subjective and objective burden. Both of these were self-report measures.

Although the sleep diaries are easy to complete, practical, cost effective and records the participant's perception of their sleep. Disadvantages of this subjective 
measurement of sleep include difficulties in recall of ties of going to bed, burden of recording information, difficulty in correlating with objective measure and inability to measure degree of day time impairment and emotional distress caused by sleep difficulties (Rodgers et al., 1993).

Although there are several studies that support the use of sleep diaries and actigraphy for examining sleep (Rodgers et al., 1993; Sedah et al., 1995; Usui et al., 1998 \& Lockley et al., 1999), these instruments are not sufficient to measure disruptions in sleep cycles. Polysomnography is the gold standard for measuring sleep, and would be the most appropriate method to examine sleep wake cycles in these parents (Jean-Louis et al., 2000). Other limitations with using wrist actigraph include trouble in discriminating sleep from quiet rest, risk of missing data due to participate removing the wrist actigraph and choosing not to wear it at all times during the study, gender, and age related differences in actigraphic data and risk of error or missing data due to actigraph malfunction (Rodgers et al., 1993).

The scoring of the CMCC instruments was tedious. Parents had to complete both time and effort for each item in the domains. Missing responses prevented calculation of total demand scores; therefore results for objective burden were not included in analysis that assessed relationships between variables.

This study also has several strengths. There are currently no published research studies examining sleep quality, fatigue, and caregiver burden of parents of children with cancer during high-dose steroid treatment. This study examined a symptom cluster within a family context and examined the symptom clusters over time. The literature supports exploring changes of symptoms over time, and exploring relationships between symptoms and investigating the effects of symptom clusters on children and parent outcomes (Gedaly-Duff et al., 2006; Dahl \& El-Sheikh, 2007). This type of study design may contribute to better understanding and better targeted health interventions for symptom clusters in children and their parents.

Both subjective and objective measures were used to capture sleep quality in the parents and their children. Both mothers and fathers were eligible to participate in this study. Although the number of male participants was low, results revealed interesting findings about fathers' experiences caring for a child with cancer and gender differences in caregiving. Caregiver burden was also examined using both objective and subjective measures. This provides a more holistic view of caregiver burden in parents.

\section{Practice Implications}

Implications for practice are discussed in this section. Implications for practice reflect the study findings related to sleep quality, fatigue, and caregiver burden in parents of children diagnosed with cancer. There were several clinically meaningful findings in this study. The results from the study indicate that parent caregivers of children with cancer have poor sleep quality in general and increased caregiver burden and fatigue 
during the time their child is receiving high-dose steroid treatment for ALL. Periodical assessments of the health status of parents of these children may need to be implemented as part of routine assessment of the ill child. These parent assessments could be incorporated at the different phases of treatment with protocols designed to include appropriate referrals and plans to support the health of the parents. Parent health should be taking on by pediatric focused health care systems to improve the outcome of the pediatric patients.

Perhaps the use of fatigue and burden instruments similar to the ones used in this study could be used as indicators for referral for parents who may need support. Findings from this study support educating parents and children before they begin high dose steroid therapy about the changes in sleep patterns and side effects of this treatment. Such educational strategies may assist in reducing caregiver burden and fatigue in the parents and fatigue and sleep disturbances in the children. Pediatric health facilities such as St. Jude Children's Research Hospital may need to explore extending the paradigm of family-centered care to include care of adults.

Higher demand scores were noted with items that included meeting emotional needs of sick child, attending medical appointments, comforting child through painful procedures, planning activities for the sick child, traveling for appointments, and disciplining the ill child. These suggest that interventions need to be aimed at addressing these items to reduce the burden of care on parent caregivers. Practice interventions also need to be aimed at improving sleep hygiene in these parents and their children with ALL. Meltzer and Mindell (2007) suggest that sleep disturbances in children can be treated which would result in improved sleep for the child and possibly improved sleep and functioning for other family members.

\section{Theoretical Implications}

The study results partially support the aspects of the Human Response Model (HRM) indicating that attributes such as effects of dexamethasone on the child's sleep quality and fatigue influence the sleep quality, fatigue, and caregiver burden of the parent caregiver. This study does not address environmental factors, which may have contributed to the parents' individual adaptation to their child's illness. Study findings provided little support for the influence of person factors such as age and gender to individual adaptation (Figure 1-1)

\section{Recommendations for Further Research}

Based on the findings of this study, additional study is needed to explore the following:

1. If there are other demographic characteristics that may contribute to parental sleep quality, caregiver burden and fatigue in these families. 
2. Fathers' perspective of caregiver burden and sleep quality when caring for a child with cancer needs further exploration because the findings of the small subgroup of fathers in this study suggest that their perspectives are somewhat different.

3. Sleep within a family context as this type study design may help in the understanding of key aspects of sleep. It is likely that social and family bonding is essential in understanding the elements of sleep (Dahl \& ElSheikh, 2007). These researchers suggest that there are some deeper issues about sleep patterns and arousals that point to the importance of family context as a conceptual framework when studying sleep.

4. Interventions that may be helpful in reducing caregiver burden, poor sleep quality and fatigue in parent caregivers of children with cancer. More specifically interventions may need to be directed at the phases of treatment protocols for cancer. There may be opportunities at the start of each phase of treatment to prepare the parents for treatment regimen and to address potential sources of burden or fatigue in parents.

5. Parental sleep quality, caregiver burden and fatigue in the context of cultural differences. There are studies that support cultural differences in caregiver burden. Little is known about cultural differences in sleep and fatigue.

\section{Summary}

In conclusion, the results of this study demonstrate the presence of significantly poor sleep quality in parents of children with ALL. It also demonstrates gender differences in caregiver burden and sleep quality in parents with children diagnosed with ALL. The literature has documented the importance of the health of the parent caregiver to the health of the ill child. Hopefully findings obtained from this study pertaining to parental caregiver burden, sleep quality, and fatigue reported by family caregivers of pediatric patients with ALL will assist healthcare providers to develop appropriate interventions that are designed to anticipate needs of caregivers and prevent errors in caregiving and future studies designed to assess such needs in parents of children with ALL. 


\section{LIST OF REFERENCES}

American Psychological Association. (2000). DSM-IV-TR . (4 ${ }^{\text {th }}$ ed., text revision). Washington, D.C.: American Psychological Association.

Anderson, K.O., Getto, C.J., Mendoza, T.R., Palmer, S.N., Wang, X.S., Reyes-Gibby, C.C., et al. (2003). Fatigue and sleep disturbance in patients with cancer patients with clinical depression and community-dwelling adults. Journal of Pain and Symptom Management, 25(4), 307-318.

Andrews, S.C. (2001). Caregiver burden and symptom distress in people with cancer receiving hospice care. Oncology Nursing Forum, 28, 1469-1474.

Bachanas, P.J., Kullgren, K.A., Schwartz, K.S., McDaniel, J.S., Smith, J., \& Nesheim, S. (2001). Psychological adjustment in caregivers of school-age children infected with HIV: Stress, coping, and family factors. Journal of Pediatric Psychology, 26, 331-342.

Bath, P.A. \& Rees, C.E. (2000). The information needs and source preferences of women with breast cancer and their family members: A review of literature published between 1988 and 1998. Journal of Advanced Nursing, 31(4), 833-41.

Becker, P.T., Chang, A., Kameshima, S. \& Bloch, M. (1991). Correlates of diurnal sleep patterns in infants of adolescents and adult single mothers. Research in Nursing $\&$ Health, 14(2), 97-108.

Belza, B.L., Henke, C.J., Yelin, E.H., Epstein, W.V., \& Gilliss, C.L. (1993). Correlates of fatigue in older adults with rheumatoid arthritis. Nursing Research, 42, 93-99.

Berger, A.M. \& Higginbotham P. (2000). Correlates of fatigue during and following adjuvant breast cancer chemotherapy: A pilot study. Oncology Nursing Forum, 27(9), 1443-1448.

Birenbaum, L.K. \& Clarke-Steffen, L. (1992). Terminal care cost in childhood cancer. Pediatric Nursing, 18(3), 285-288.

Black, M.M., Papas, M.A., Hussey, J.M., Dubowitz, H., Kotch, J.B. \& Starr, R.H. Jr. (2002). Behavioral problems among preschool children born to adolescent mothers: Effects of maternal depression and perceptions of partner relationships. Journal of Clinical Child Psychology, 31(1), 16-26.

Boergers, J., Hart, C., Owens, J., \& Streisand, R., (2007). Child sleep disorders: Associations with parental sleep duration and daytime sleepiness. Journal of Family Psychology, 21(1), 88-94. 
Boman, K., Lindahl, A. \& Bjork, O. (2003). Disease-related distress in parents of children with cancer at various stages after the time of diagnosis. Acta Oncologica, 42(2), 137-146.

Boman, K., Viksten, J., Kogner, P. \& Samuelsson, U. (2004). Serious illness in childhood: The different threats of cancer and diabetes from a parent perspective. Journal of Pediatrics, 145, 373-379.

Burns, N. \& Grove, S. (2001). The practice of nursing research conduct, critique and utilization. $\left(4^{\text {th }}\right.$ ed.). Philadelphia, PA: W.B. Saunders Company.

Cafferta, G.L. \& Stone, R. (1989). The caregiving role: Dimensions of burden and benefits. Compre Gterontol, 3 Suppl, 57-64.

Carter, P.A. (2002). Caregivers' descriptions of sleep changes and depressive symptoms. Oncology Nursing Forum, 29(9), 1277-1283.

Carter, P.A. (2003). Family caregivers' sleep loss and depression over time. Cancer Nursing, 26(4), 253-259.

Caserta, M.S., Lund, D.A. \& Wright, S.D. (1996). Exploring the caregiver burden inventory (CBI): Further evidence for a multidimensional view of burden. The International Journal of Aging and Human Development, 43(1), 21-34.

Chokroverty, S. (1999). An overview of sleep. In Sleep disorders medicine basic science, technical considerations and clinical aspects ( $2^{\text {nd }}$ ed.). Woburn, MA: Butterworth-Heinemann.

Chou, K.R. (2000).Caregiver burden: A concept analysis. Journal of Pediatric Nursing, 15(6), 398-407.

Cornman, B. J. (1993). Childhood cancer: Differential effects on the family members. Oncology Nursing Forum, 20, 1559-1566.

Cotton, S. \& Richdale, A. (2005). Brief report: Parental descriptions of sleep problems in children with autism, Down Syndrome, and Prader-Willi syndrome. Research in Developmental Disabilities, pp. 1-11.

Craft, A.W. (2000). Childhood cancer - mainly curable so where next? Acta Paediat, 89, $386-92$.

Cummings, S.T. (1976). The impact of the child's deficiency on the father: A study of fathers of mentally retarded and of chronically ill children. American Journal of Orthopsychiatry, 46, 246-55. 
Dahl, R.E., \& El-Sheikh, M. (2007). Considering sleep in a family context: Introduction to the special issue. Journal of Family Psychology, 21, 1-3.

Dalquist, L.M., Czyzewski, D.I. \& Jones, C.L. (1996). Parents of children with cancer: A longitudinal study of emotional distress, coping style, and marital adjustment two and twenty months after diagnosis. Journal of Pediatric Psychology, 21, 541554.

Davies, B., Gudmundsdottir, M., Worden, B., Orloff, S., Sumner, L. \& Brenner, P. (2004). "Living in the dragon's shadow" fathers' experiences of a child's lifelimiting illness. Death Studies, 28(2), 111-135.

Deeken, J.F., Taylor, K.L., Mangan, P., Yabroff, K.R., \& Ingham, J.M. (2003). Care for the caregivers: A review of self-report instruments developed to measure the burden, needs, and quality of life of informal caregivers. Journal of Pain and Symptom Management, 26, 922-953.

Dilworth-Anderson, P., Williams, I.C., \& Gibson, B.E. (2002). Issues of race, ethnicity, and culture in caregiving research: A 20-year review (1980-2000). Gerontologist, $42,237-272$.

Donelan, K., Hill, C.A., Hoffman, C., Scoles, K., Feldman, P.H., Levine, C., et al. (2002). Challenged to care: informal caregivers in a changing health system. Health Affairs (Millwood), 21, 222-231.

Drigan, R., Spirato, A. \& Gelber, R.D. (1992). Behavioral effects of corticosteroids in children with acute lymphoblastic leukemia. Medical and Pediatric Oncology, 20, 13-21.

Drotar, D. (1997). Relating parent and family functioning to the psychological adjustment of children with chronic health conditions: What have we learned? What do we need to know? Journal of Pediatric Psychology, 22, 149-165.

Dzurec, L.C., Hoover, P.M. \& Fields, J. (2002). Acknowledging unexplained fatigue of tired women. Journal of Nursing Scholarship, 34(1), 41-46.

Earle, E.A., Clarke, S.A., Eiser, C. \& Sheppard, L. (2007). 'Building a new normality': Mothers' experiences of caring for a child with acute lymphoblastic leukaemia. Child:Care Health and Development, 33(2), 155-160.

Eckerberg, B. (2004). Treatment of sleep problems in families with young children: Effects of treatment on family well-being. Acta Paediatrica, 93, 126-134.

Eidelman, D. (2002). What is the purpose of sleep? Medical Hypotheses, 58, 120-122. 
Eiser, C., Davies, H., Jenney, M., Stride, C. \& Glaser, A. (2006). HRQOL implications of treatment with dexamethasone for children with acute lymphoblastic leukemia (ALL). Pediatric Blood \& Cancer, 46(1), 35-39.

Eiser, C., Eiser, J.R., \& Stride, C.B. (2005). Quality of life in children newly diagnosed with cancer and their mothers. Health and Quality of Life Outcomes, 28, p. 29.

Floyd, J., Medler S.M., Ager, J.W. \& Janisse, J.J. (2000). Age-related changes in initiation and maintenance of sleep: A meta-analysis. Research in Nursing \& Health, 23(2), 106-117.

Garwich, A.W., Patterson, J., Bennett, F.C., \& Blum, R.W. (1995). Breaking the news: How families first lean about their child's chronic condition. Archives of Pediatrics \& Adolescent Medicine Med, 149, 991-997.

Gattuso, J., Hinds, P., Tong, X. \& Srivastava, K. (2006). Monitoring child and parent refusals to enroll in clinical research protocols. Journal of Advanced Nursing, 53(3), 319-326.

Gau, S.S. \& Merikangas, K.R. (2004). Similarities and differences in sleep-wake patterns among adults and their children. Sleep, 27(2), 299-304.

Gay, C.L., Lee, K.A., \& Lee, S.Y. (2004). Sleep patterns and fatigue in new mothers and fathers. Biological Research for Nursing, 5, 311-318.

Gedaly-Duff, V., Lee, K.A., Nail, L., Nicholson, H.S., \& Johnson, K.P. (2006). Pain, sleep disturbance, and fatigue in children with leukemia and their parents: A pilot study. Oncology Nursing Forum, 33, 641-646.

Gibson, F., Garnett, M., Richardson, A., Edwards, J., \& Sepion, B. (2005). Heavy to carry: a survey of parents' and healthcare professionals' perceptions of cancerrelated fatigue in children and young people. Cancer Nursing, 28, 27-35.

Glaus, A. (1993). Assessment of fatigue in cancer and non-cancer patients and in healthy individuals. Supportive Care in Cancer, 1(6), 305-315.

Goll, J.C., \& Shapiro, C.M. (2006). Sleep disorders presenting as common pediatric problems, Canadian Medical Association Journal, 174, 617-619.

Grigg-Damberger, M. (2004). Neurologic disorders masquerading pediatric sleep problem. Pediatric Clinic of North America 51, 89-115.

Harris, J.C., Carel, C.A., Rosenberg, L.A., Paramjit, J. \& Leventhal, B.G. (1986). Intermittent high dose corticosteroid treatment in childhood cancer. Journal of American Academy of Child Psychology, 25, 120-124. 
Heitkemper, M., Levy, R. \& Jarrett, M. (1995). Interventions for irritable bowel syndrome: A nursing model. Gastrointestinal Nursing, 18, 224-230.

Heitkemper, M. \& Shaver, J. (1989). Nursing research opportunities in enteral nutrition. Nursing Clinics of North America, 24, 415-426.

Heussler, H.S. (2005). Common causes of sleep disruption and daytime sleepiness: Childhood sleep disorders II. Medical Journal of Australia, 182, 484-489.

Hinds, C. (1985). The needs of families who care for patients with cancer at home: Are we meeting them? Journal of Advanced Nursing, 10(6), 575-581.

Hinds, P.S., Birenbaum, L.K., Clarke-Steffen, L., et al. (1996). Coming to terms: Parents response to a first cancer recurrence in their child. Nursing Research, 48, 148-153.

Hinds, P.S., Hockenberry, M.J., Gattuso, J.S., Kumar Srivastava, D., Tong, X., Jones, H., et al. (2007) Dexamethasone alters sleep and fatigue in pediatric patients with acute lympoblastic leukemia. Cancer, 110 (10), 2321-2330.

Hovey, J.K. (2005). Fathers parenting chronically ill children: Concerns and coping strategies. Issues in Comprehensive Pediatric Nursing, 28(2), 83-95.

Igarashi, S., Manabe, A., Ohara, A., Kumagai, M., Saito, T., Okimoto, Y., et al. (2005). No advantage of dexamethasone over prednisolone for the outcome of standardand intermediate-risk childhood acute lymphoblastic leukemia in the Tokyo Children's Cancer Study Group L95-14 protocol. Journal of Clinical Oncology 23, 6489-6498.

Iwasaki, M. (2005). Interventional study on fatigue relief in mothers caring for hospitalized children--effect of massage incorporating techniques from oriental medicine. Kurume Medical Journal, 52, 19-27.

James, K. \& Keegan-Wells, D. (2002). The care of my child with cancer: Parent's perceptions of caregiving demands. Journal of Pediatric Oncology Nursing. 19(6), 218-228.

Jean-Louis, G., Kripke, D.F. \& Ancoli-Isreal, S. (2000). Sleep quality and well- being. Sleep 23(8), 115-121.

Jean-Louis, G., Kripke, D.F., Mason W.J., Elliot J.A. \& Youngstedt, S.D. (2000). Sleep estimation from wrist movement quantified by different actigraphic modalities. Neuroscience Methods, 105(2), 185-191.

Jean-Louis, G., Mendlowicz, M.V., Gizycki, H.V., Zizi, F., \& Nunes, J. (1999). Assessment of physical activity and sleep by actigraphy: Examination of gender 
differences. Journal of Women's Health and Gender-Based Medicine. 8(8), 1113-1117.

Jensen, S. \& Given, B.A. (1991). Fatigue affecting family caregivers of cancer patients. Canadian Nursing, 14(4):181-7.

Kaspers, G.J., Pieters, R., \& Veerman, A.P. (1997). Glucocorticoid sensitivity and resistance in childhood leukemia. In R. Pieters, G. Kaspers \& A. Veerman (eds). Drug resistance in leukemia and lymphoma II. The Netherlands: Harwood Academic Publishers, 95-103.

Katz, S. (2002). When the child's illness is life threatening: Impact on the parents. Pediatric Nursing, 28, 453-463.

Keegan-Wells, D., James, K., Stewart, J.L. et al. (2002). The care of my child with cancer: A new instrument to measure caregiving demand in parents of children with cancer. Journal Pediatric Nursing, 17(3), 201-210.

Knafl, K.A., \& Deatrick, J.A. (1986). How families manage chronic conditions: An analysis of the concept of normalization. Research in Nursing \& Health, 9, 215222.

Knafl, K.A., Gailo, A.M., Zoeller, L. \& Breitmayer, B. (1993). Family response to a child's chronic illness: A description of major defining themes. In S.G. Funk (ed), Key Aspects of Caring for the Chronically Ill: Hospital and Home, New York: Springer, 290-303.

Knafl, K.A. \& Zoeller, L. (2000). Childhood Chronic Illness: A comparison of mothers' and fathers' experiences. Journal of Family Nursing, 6, 287-302.

Krupp, L., Jandorf, L., Coyle, P. \& Mendelson, W. (1993). Sleep disturbance in chronic fatigue syndrome. Journal of Psychosomatic Research, 37, 325-331.

Lahteenmaki, P.M., Sjoblom, J., Korhonen, T., \& Salmi, T.T. (2004). The life situation of parents over the first year after their child's cancer diagnosis. Acta Paediatrica, 93, 1654-1660.

Landis, C.A., (2002) Sleep and methods of assessment. Nursing Clinics of North America, 37(4), 583-97.

Lansky S.B., Cairns, N.U., Clark, G.M. et al. (1979). Childhood cancer: Nonmedical costs of the illness. Cancer, 43, 403-408.

Lauderdale, D.S., Knutson, K.L., Yan, L.L., Rathouz, P.J., Hulley, S.B., Sidney, S. et al. (2006). Objectively measured sleep characteristics among early-middle-aged adults: The CARDIA study. American Journal of Epidemiology, 164, 5-16. 
Lee, S.Y., Lee, K.A., Rankin, S.H., Weiss, S.J., \& Alkon, A. (2007). Sleep disturbance, fatigue, and stress among Chinese-American parents with ICU hospitalized infants. Issues Mental Health Nursing, 28, 593-605.

Leech, J.A., Onal, E., Dulberg C., et al. (1988). A comparison of men and women with occlusive sleep apnea syndrome. Chest, 94, 983-989.

Leininger, M. (1991). Culture care diversity to universality: a theory of nursing: New York: National League for Nursing Press.

Liang, H.F. (2002). Understanding culture care practices of caregivers of children with cancer in Taiwan. Journal Pediatric Oncology Nursing, 19, 205-217.

Lichstein, K.L., Means, M.K., Noe, S.L. \& Aguillard, R.N. (1997). Fatigue and sleep disorders. Behaviour Research and Therapy, 35(8), 733-740.

Litwin, M. (Eds.) (1995). How to measure survey reliability and validity. (pp. 21-31). Thousand Oaks, CA: Sage Publications, Inc.

Lockley S.W., Skene, D.J. \& Arendt, J. (1999). Comparison between subjective and actigraphic measurement of sleep and sleep rhythms. Journal of Sleep Research, $8(3), 175-183$.

Lutzky, S.M. \& Knight. B.G. (1994). Explaining gender differences in caregiver distress: The roles of emotional attentiveness and coping styles. Psychology \& Aging, 9(4), 513-519.

Marquis, P. (1996). Sleep disturbance: A component of health status. PharmacoEconomics, 10(1), 25-28.

Martin, J., Hiscock, H., Hardy, P., Davey, B., \& Wake, M. (2007). Adverse associations of infant and child sleep problems and parent health: An Australian population study. Pediatrics, 119, 947-955.

Martinson, I.M., Leavitt, M., Liu, C., Armstrong, V., Hornberger, L., Zhang, J. \& Han, X. (1999). Comparing of Chinese and Caucasian families caregiving to children with cancer at home: Part I. Journal of Pediatric Nursing, 14(2), 99-109.

McCorkle, R., Huges, L.C., Hodgson, N.A., Muller, P. \& Robinson, L.A. (2000). Information needs of elderly postsurgical cancer patients during the transition from the hospital to home. Journal of Nursing Scholarship, 32(1), 25-30.

McCubbin, H.I. \& Patterson, J.M. (1983). Family stress and adaptation to crisis: A Double ABCX Model of family behavior. In D. Olsen \& B. Miller (Eds.), Family studies review year-book. (p. 87). Beverly Hills, CA: Sage. 
McGarry, J. \& Arthur, A. (2001). Informal caring in late life: A qualitative study of the experiences of older careers. Journal Advanced Nursing, 33(2), 182-189.

Mcrath, P. (2001) Identifying support issues of parents of children with leukemia. Cancer Practice, 9, 198-205.

Meltzer, L.J. \& Mindell, J.A. (2006). Sleep and sleep disorders in children and adolescents. Psychiatric Clinics of North America, 29, 1059-1076.

Meltzer, L.J. \& Mindell, J.A. (2007). Relationship between child sleep disturbances and maternal sleep, mood, and parenting stress: A pilot study. Journal of Family Psychology, 21, 67-73.

Mendoza, T.R., Wang, X.S., Cleeland, C.S., Morrissey, M., Johnson, B.A., Wendt, J.K. \& Huber, S.L. (1999). The rapid assessment of fatigue severity in cancer patients: Use of the brief fatigue inventory. Cancer 85(5), 1186-1196.

Miles, M.S., D'Auria, J.P., Hart, E.M., Sedlack, D.A. \& Watral, M.A. (1993). Parental role alterations experienced by mothers of children with a life-threatening chronic illness. In S.G. Funk, E.M. Tornquist, M.T. Champagne \& R.A. Wiese (Eds.) Key Aspects of Caring for the Chronically Ill (pp. 281-289). New York: Springer Publishing, 281-289.

Miles, M.S. (2003). Parents of children with chronic health problems: Programs of nursing research and their relationship to nursing science. In J. Fitzpatrick (Ed.), The annual review of nursing research: Research on child health and pediatric issues. (Vol. 21). New York: Springer Publishing Company.

Mindell, J.A. \& Durand, V.M. (1993). Treatment of childhood sleep disorders: Generalizations across disorders and effects on family member. Journal of Pediatric Psychology, 18(6), 731-750.

Mitchell, C.D., Richards, S.M., Kinsev, S.E., Lilleyman, J., Vora, A., \& Eden, T.O. (2005). Benefit of dexamethasone compared with prednisolone for childhood acute lymphoblastic leukaemia: results of the UK Medical Research Council ALL97 randomized trial, British Journal of Haematology, 129, 734-745.

Morriss, R., Sharpe, M., Sharpley, A. Cowen, P., Hawton, K. \& Morris, J. (1993). Abnormalities of sleep in patients with chronic fatigue. British Medical Journal, 306, 1161-1164.

Moser, N.J., Phillips, B., Guthrie, G. \& Barnett G. (1996). Effects of dexamethasone on sleep. Basic \& Clinical Pharmacology \& Toxicology, 79(2), 100-102. 
National Sleep Foundation (2004a). Sleep in America poll. Retrieved January 31, 2006, from the World Wide Web: http://www.sleepfoundation.org.

National Sleep Foundation (2004b). Final Report: 2004 Sleep in American Poll. Retrieved June 23, 2006, from the World Wide Web: http://www.sleepfoundation. org/_content/ hottopics/2004 SleepPollFinalReport.pdf.

Navaie-Waliser, M., Feldman, P.H., Gould, D.A., Levine, C., Kuerbis, A.N. \& Donelan, K. (2002). When the caregiver needs care: the plight of vulnerable caregivers. American Journal of Public Health, 92(3), 409-413.

Nijboer, C., Tempelaar, R., Triemstra, M. et al. (2001). The role of social and psychologic resources in caregiving of cancer patients. Cancer, 91(5), 10291039.

Nijboer, C., Triemstra, M., Tempelaar, R. et al. (1999). Measuring both negative and positive reactions to giving care to cancer patients: Psychometric qualities of the Caregiver Reaction Assessment (CRA). Social Science Medical, 48, 1259-1269.

Oberst, M.T., Thomas, S.E., Gass, K.A. \& Ward, S.E. (1989). Caregiving demands and appraisal of stress among family caregivers. Cancer Nursing, 12(4), 209-215.

Ohayon, M., Carskadon, M.A., Guilleminault, C. et al. (2004) Meta-analysis of quantitative sleep parameters from childhood to old age in healthy individuals: Developing normative sleep values across the human lifespan. Sleep, 27, 12551273.

Ohayon, M.M. \& Shapiro, C.M. (2000). Sleep and fatigue. Seminars in Clinic Neuropsychiatry, 5(1), 56-57.

Patterson, J.M., \& Garwick, A.W. (1994). Levels of meaning in family stress theory. Family Process Journal, 33, 287-304.

Pearlin, L.I., Mullan, J.T., Semple, S.J. \& Skatt, M.M. (1990). Caregiving and the stress process: an overview of concepts and their measures. Gerontologist 30, 583-594.

Peck, B. \& Lillibridge, J. (2005). Normalization behaviors of rural fathers living with chronically-ill children: An australian experience. Journal of Child Health Care, 9, 31-45.

Phipps, S., Dunavant, M., Lensing, S., \& Rai, S.N. (2004). Patterns of distress in parents of children undergoing stem cell transplantation. Pediatric Blood \& Cancer, 43, 267-274. 
Pilcher, J.J., Ginter, D.R., \& Sadowsky, B. (1997). Sleep quality versus sleep quantity: relationship between sleep and measures of health, well-being and sleepiness in college students. Journal of Psychosometric Research, 42, 583-596.

Polit, D. \& Hungler, B. (1991). Nursing research: Principles and methods. (4 ${ }^{\text {th }}$ ed.). Philadelphia, PA: J.B. Lippincott Company.

Pradeep, R., Prakash, P., \& Shah H. (2004). Psychopathology and coping in parents of chronically ill children. The Indian Journal of Pediatrics, 71(8), 695-699.

Pui, C. (2006). Childhood Leukemias. ( $2^{\text {nd }}$ ed.). New York: Cambridge University Press.

Pui, C. \& Evan, W. (2007). Treatment of acute lymphoblastic leukemia. The New England Journal of Medicine, 354(2), 166-178.

Rahman, A., Harrington, R. \& Bunn, J. (2002). Can maternal depression increase infant risk of illness and growth impairment in developing countries? Child: Care, Health and Development, 28(1), 51-56.

Raina, P., O'Donnell, M., Schwellnus, H., Rosenbaum, P., King, G., Brehaut, J., Russell, D., Swinton, M., King, S., Wong, M., Walter, S. \& Wood, E. (2004). Caregiving process and caregiver burden: Conceptual models to guide research and practice. BMC Pediatrics, 4(1).

Richards, K.C. (1996). Sleep promotion. Critical Care Nursing Clinics of North America, 8, 39-52.

Riter, S. \& Willis, L. (2004). Sleep wars: research and opinion. Pediatric Clinics of North America, 51, 1-13.

Robinson, A.M. \& Richdale, A.L. (2004). Sleep problems in children with an intellectual disability: Parental perceptions of sleep problems, and views of treatment effectiveness. Child: Care, Health \& Development 30(2), 139-150.

Rodgers, A.E., Caruso, C.C. \& Aldrich, M. (1993). Reliability of sleep diaries for assessment of sleep/wake patterns. Nursing Research, 42(6), 368-372.

Rodrigues, N. \& Patterson, J.M. (2007). Impact of severity of a child's chronic condition on the functioning of two-parent families. Journal of Pediatric Psychology, 32, 417-426.

Sadeh, A., Hauri, P.J. Kripke, C.F., et al. (1995). The role of actigraphy in the evaluation of sleep disorders. Sleep, 18, 288-302. 
Schofield, H.L., Murphy, B., Herman, H.E., Bloch, S. \& Singh, B. (1997). Family caregiving: Measurement of emotional well-being and various aspects of the caregiving role. Psychological Medicine, 27(3), 647-657.

Schumacher. K.L., Dodd, M.J. \& Paul, S.M. (1993). The stress process in family caregivers of persons receiving chemotherapy. Research in Nursing \& Health, $16,395-404$.

Sepa, A., Frodi, A., \& Ludvigsson, J. (2004). Psychosocial correlates of parenting stress, lack of support and lace of confidence/security. Scandinavian Journal of Psychology, 45, 169-179.

Shapiro, C.M. (1998). Fatigue: How many types and how common? Journal of Psychosomatic Research 45(1 Spec No), 1-3.

Shaver, J. (2002). Women and sleep. Nursing Clinics of North America, 37, 707-718.

Sheeran, T., Marvin, R. \& Pianta, R. (1996). Mothers' resolution of their child's diagnosis and self-reported measures of parenting stress, marital relations and social support. Journal of Pediatric Psychology 22(2), 197-212.

Steele, R.G., Long, A., Reddy, K.A., Phipps, S., \& Luhr, M. (2003). Changes in maternal distress and child-rearing strategies across treatment for pediatric cancer. Journal of Pediatric Psychology, 28, 447-52.

Steen, G. \& Mirro, J. (2000). Childhood cancer: A handbook from St. Jude Children's Research Hospital with contributions from St. Jude clinicians and scientists. Cambridge, MA: Persus Publishing.

Stevens, B., Croxford, R., McKeever, P., Yamada, J., Booth, M., Daub, S., Gafni, A., Gammon, J. \& Greenberg, M. (2005). Hospital and home chemotherapy for children with leukemia: A randomized cross-over study. Pediatric Blood \& Cancer, pp. 1-8.

Stewart, M.J, Ritchie, J.A., McGrath, P., Thompson, D. \& Bruce, B. (1994). Mothers of children with chronic conditions: Supportive and stressful interactions with partners and professionals regarding caregiving burdens. Canadian Journal of Nursing Research, 26, 61-82.

Stuart, F.A., Segal, T.Y. \& Keady, S. (2005). Adverse psychological effects of corticosteroids in children and adolescents. Archives of Disease in Childhood, 90(5), 500-506.

Sullivan-Bolyai, S., Sadler, L., Knafl, K.A., \& Gilliss, C.L. (2003). Great expectations: A position description for parents as caregivers: Part I. Pediatric Nursing, 29, 457-461. 
Svavarsdottir, E.K. (2005a). Caring for a child with cancer: A longitudinal perspective. Journal of Advanced Nursing 50(2), 153-161.

Svavarsdottir, E.K. (2005b). Gender and emotions: Icelandic parents experiencing childhood cancer. International Journal of Nursing Studies 42, 531-538.

Svavarsdottir, E.K. (2005c). Surviving childhood cancer: parents' perceptions of their child's health. Journal of Pediatric Oncology Nursing, 22, 80-88.

Talbot, L. (1995). Principles and practice of nursing research. St. Louis, MO: Mosby.

Thoma, M.E., Hockenberry-Eaton, M. \& Kemp, V. (1993). Life change events and coping behaviors in families of children with cancer. Journal of Pediatric Oncology Nursing, 10, 105-111.

Tidwell, J. Unpublished Data. Measuring parental caregiver burden in parents of Children with Acute Lymphocytic Leukemia.

Tidwell, J. (2003). Seeking a Standard for Measuring Parental Sleep. Poster session presented at the University of Tennessee Health Science Center Annual Alumni Research Conference Memphis Tennessee.

Usui, A., Ishizuka, Y., Obinata, I., Okado,T., Fukuzawa, H. \& Kanba, S. (1998). Validity of sleep log compared with actigraphic sleep-wake state. Psychiatry and Clinical Neurosciences. 52(2), 161-163.

Usui, A., Ishizuka, Y., Obinata, I., Okado,T., Fukuzawa, H. \& Kanba, S. (1999). Validity of sleep log compared with actigraphic sleep-wake state II. Psychiatry and Clinical Neurosciences. 53(2), 183-184.

Van Brunt, D., Riedel, B.W. \& Lichstein, K.L. (1996). Validation of sleep diary. In Van Hasset and Hersen (Eds) Sourcebook of psychological treatment manuals of adults disorders (pp. 539-566) New York, NY: Plenum Press.

Van Riper, M. (2000). Family variables associated with well-being in families of children with Down Syndrome. Journal of Family Nursing, 6, 267-286.

Walsh, S.M., Martin, S.C. \& Schmidt, L.A. (2004). Testing the efficacy of a creative-arts intervention with family caregivers of patients with cancer. Journal of Nursing Scholarship, 36(3), 214-219.

Wang, X.I., Janjan, N.A., Guo, H., Johnson, B.A., Engstrom, M.C., Crane, C.H., et al. (2001). Fatigue during preoperative chemoradiation for resectable rectal cancer. Cancer 92 (6 Suppl), 1725-1732. 
Ware, J.E., Snow, K.K., Kosinski, M. \& Gandek, M.S. (1993). SF-36 health survey manual and interpretation guide. Boston, MA: Nimrod Press.

Watanabe, T.K., Sylvester, C.E. \& Manaligod, J.M. (1994). Mania or panic associated with dexamethasone chemotherapy in adolescents. Journal of Adolescent Health, 15(4), 345-347.

Wiener, L.S., Vasquez, M.J. \& Battles, H.B. (2001). Brief report: fathering a child living with HIV/AIDS: Psychosocial adjustment and parenting stress. Journal of Pediatric Psychology, 26, 353-358.

Wiggs, L. \& Stores, G. (1998). Behavioural treatment for sleep problems in children with sever learning disabilities and challenging daytime behavior: Effect on sleep patterns of mother and child. European Sleep Research Society, 7, 119-126.

Wingo, P.A., Tong, T. \& Bolden, S. (1995). Cancer statistics, 1995. CA: A Cancer Journal for Clinicians, 45(2), 127-128.

Yates, M.E., Tennstedt, S. \& Chang, B.H. (1999). Contributors to and mediators of psychological well-being for informal caregivers. Journal of Gerontology Series B: Psychological Sciences and Social Sciences, 54(1), 12-22.

Yeh, C.H. (2002). Gender differences of parental distress in children with cancer. Journal of Advanced Nursing 38(6), 598-606.

Yellen, S.B., Cella, D.F., Webster, K., Blendowski, C. \& Kaplan, E. (1997) Measuring fatigue and other anemia-related symptoms with the functional assessment of cancer therapy (FACT) measurement system. Journal of Pain and Symptom Management, 13(2), 63-74.

Zoorob, R.J. \& Cender, D. (1998). A different look at corticosteroids. American Family Physician, 58(2), 443-450. 
APPENDICES 
APPENDIX A

THE CARE OF MY CHILD WITH CANCER INSTRUMENT (CMCC) 


\section{THE CARE OF MY CHILD WITH CANCER}

PARENTS/GUARDIANS PUT TIME AND EFFORT INTO TAKING CARE OF THEIR CHILD WITH CANCER. WE WANT TO BETTER UNDERSTAND HOW MUCH TIME AND EFFORT CERTAIN CARE-GIVING TASKS REQUIRE. PLEASE INDICATE BELOW THE AMOUNT OF TIME AND THE AMOUNT OF EFFORT DURING THE PAST WEEK THAT

THESE TASKS HAVE REQUIRED OF YOU.

\section{PHYSICAL CARE}

1. PREPARING AND GIVING MEDICINES, FLUIDS AND TPN (NUTRITION) INTRAVENOUSLY (IV) (PREPARATION INCLUDES: TUBING, PUMPS, DRAWING UP MEDICATIONS)

\begin{tabular}{ll} 
TIME & $>5$ HOURSWEEK \\
& $>2$-5 HOURS WEEK \\
& $1-2$ HOURSNEEK \\
& $<1$ HOURNEEK \\
\hline & DID NOT DO
\end{tabular}

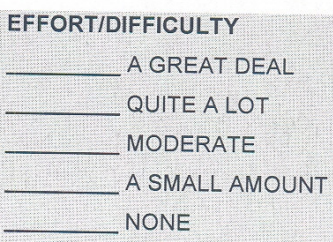

PREPARING AND GIVING MEDICINE AS A SHOT IN THE MUSCLE (IM) OR UNDER THE SKIN (SQ) (THIS INCLUDES: DRAWING UP MEDICATIONS, APPLYING EMLA CREAM).

\begin{tabular}{|c|c|}
\hline TIME & EFFORTIDIFFICULTY \\
\hline$\longrightarrow>5$ HOURMEEK & A GREAT DEAL \\
\hline$>2-5$ HOURSIWEEK & QUITE A LOT \\
\hline _-2 HOURSWEEK & - MODERATE \\
\hline$-<1$ HOURMEEK & A SMALL AMOUNT \\
\hline$\longrightarrow$ DID NOT DO & $\longrightarrow$ NONE \\
\hline
\end{tabular}

3. PREPARING AND GIVING MEDICATIONS BY MOUTH (EXAMPLES INCLUDE: BREAKING UP PILLS,
DISGUISING TASTE, ETC.)

\begin{tabular}{|c|c|}
\hline TIME & EFFORTIDIFFICULTY \\
\hline$\longrightarrow>5$ HOURMEEK & $\longrightarrow$ A GREAT DEAL \\
\hline$\longrightarrow 2-5$ HOURSMEEK & QUITE A LOT \\
\hline 1-2 HOURS WEEK & - MODERATE \\
\hline$\longrightarrow<1$ HOUR/WEEK & — A SMALL AMOUNT \\
\hline — DID NOT DO & $\longrightarrow$ NONE \\
\hline
\end{tabular}


4. PREPARING AND GIVING CATHETER FLUSHES

\begin{tabular}{|c|c|}
\hline TIME & EFFORT/DIFFICULTY \\
\hline$>5$ HOUR/WEEK & $\longrightarrow$ A GREAT DEAL \\
\hline$>2-5$ HOURSIWEEK & Q QUITE A LOT \\
\hline 1-2 HOURS/WEEK & MODERATE \\
\hline < 1 HOURNEEK & _ A SMALL AMOUNT \\
\hline DID NOT DO & NONE \\
\hline
\end{tabular}

5. CHANGING THE DRESSING ON YOUR CHILD'S CATHETER (I.E., BROVIAC, HICKMAN, PORT, ETC.) AND CHANGING CATHETER CAP(S).

\begin{tabular}{ll} 
TIME & EFFORTIDIFFICULTY \\
\hline$>5$ HOURMEEK & A GREAT DEAL \\
$>$ & $2-5$ HOURSNWEK \\
\hline 1-2 HOURSWWEK & QUITE A LOT \\
< & MODERATE HOURNEEK \\
\hline DID NOT DO & A SMALL AMOUNT \\
\hline
\end{tabular}

6. MANAGING SIDE EFFECTS OF CANCER OR ITS TREATMENT (EXAMPLES INCLUDE: VOMITING, MOUTH SORES, DIARRHEA, FREQUENT VOIDING/DIAPER CHANGING).

\begin{tabular}{|c|c|}
\hline TIME & EFFORT/DIFFICULTY \\
\hline$>5$ HOURMEEK & A GREAT DEAL \\
\hline$>2-5$ HOURS/WEEK & Q QUITE ALOT \\
\hline — 1-2 HOURS WEEK & _ MODERATE \\
\hline$<1$ HOURMEEK & - A SMALL AMOUNT \\
\hline DID NOT DO & $\longrightarrow$ NONE \\
\hline
\end{tabular}

7. KEEPING YOUR CHILD COMFORTABLE AND WITHOUT PAIN.

\begin{tabular}{lll} 
TIME & $>5$ HOURNWEK & EFFORTIDIFFICULTY \\
\hline & $>2-5$ HOURS/WEEK & A GREAT DEAL \\
& 1-2 HOURSNWEK & QUITE A LOT \\
& $<1$ HOURNWEK & MODERATE \\
\hline DID NOT DO & A SMALL AMOUNT \\
\hline
\end{tabular}


8. MANAGING OTHER CHILDHOOD ILLNESSES FOR YOUR CHILD WITH CANCER (EXAMPLES INCLUDE: COLD, FLU, EAR INFECTIONS, OTHER).

$\begin{array}{ll}\longrightarrow & >5 \text { HOURNWEK } \\ & >2-5 \text { HOURSNEEK } \\ & 1-2 \text { HOURSWEEK } \\ & <1 \text { HOURWEEK } \\ & \text { DID NOT DO }\end{array}$

EFFORTIDIFFICULTY
A GREAT DEAL
QUITEA LOT
MODERATE
A SMALL AMOUNT
NONE

9. MANAGING UNEXPECTED EVENTS RELATED TO YOUR CHILD'S ILLNESS (EXAMPLES INCLUDE: ADMISSION FOR FEVER, UNSCHEDULED APPOINTMENT FOR BLOOD TRANSFUSION, CHANGES IN TREATMENT SCHEDULE BECAUSE OF LOW BLOOD COUNTS).

\begin{tabular}{|c|c|}
\hline TIME & EFFORTIDIFFICULTY \\
\hline — $>5$ HOURNEEK & A GREAT DEAL \\
\hline$>2-5$ HOURS/WEEK & _ QUITE A LOT \\
\hline - 1-2 HOURSMEEK & MODERATE \\
\hline — $<1$ HOURMVEK & A SMALL AMOUNT \\
\hline DID NOT DO & NONE \\
\hline
\end{tabular}

10. ADDITIONAL HOUSEHOLD TASKS RELATED TO YOUR CHILD'S ILLNESS (EXAMPLES INCLUDE CLEANING AND MAINTENANCE OF EQUIPMENT, ETC.).

\begin{tabular}{|c|c|}
\hline TIME & EFFORT/DIFFICULTY \\
\hline$\longrightarrow 5$ HOURNEEK & A GREAT DEAL \\
\hline > 2-5 HOURS/WEEK & _ QUITE A LOT \\
\hline 1-2 HOURSNWEEK & MODERATE \\
\hline$Z_{1}<1$ HOURNEEK & A SMALL AMOUNT \\
\hline DID NOT DO & NONE \\
\hline
\end{tabular}

11. COORDINATING, ARRANGING, AND MANAGING MEDICAL SERVICES (EXAMPLES INCLUDE: SCHEDULING APPOINTMENTS, LOCATING EQUIPMENT AND NEGOTIATING SERVICES).

\begin{tabular}{|c|c|}
\hline TIME & EFFORT/DIFFICULTY \\
\hline$>5$ HOURMEEK & A GREAT DEAL \\
\hline$>2-5$ HOURSIWEEK & — QUITE A LOT \\
\hline 1-2 HOURSMEEK & _ MODERATE \\
\hline - $<1$ HOURMEEK & A SMALL AMOUNT \\
\hline DID NOT DO & NONE \\
\hline
\end{tabular}


12. TRAVEL TO AND FROM THE HOSPITAL FOR MEDICAL CARE.

\begin{tabular}{lll} 
TIME & $>5$ HOUR/WEEK & EFFORT/DIFFICULTY \\
& $>2$-5 HOURS WEEK & A GREAT DEAL \\
\hline & 1-2 HOURSWWEK & QUITE A LOT \\
& $<1$ HOURMEEK & MODERATE \\
\hline DID NOT DO & A SMALL AMOUNT \\
\hline
\end{tabular}

13. TIME SPENT AT THE HOSPITAL FOR APPOINTMENTS (EXAMPLES INCLUDE: ONCOLOGY, NEUROLOGY, RADIATION ONCOLOGY, SURGERY CLINIC, SCANS, AND OTHER TESTS).

\begin{tabular}{ll} 
TIME & \multicolumn{1}{c}{ EFFORTIDIFFICULTY } \\
\hline$>5$ HOURMEEK & A GREAT DEAL \\
$>2-5$ HOURS/WEEK & QUITEA AOT \\
$1-2$ HOURSWWEK & MODERATE \\
$<1$ HOURNWEK & A SMALL AMOUNT \\
\hline DID NOT DO & NONE
\end{tabular}

\section{EMOTIONAL CARE}

14. PROVIDING EMOTIONAL SUPPORT FOR YOUR CHILD WITH CANCER

\begin{tabular}{lll} 
TIME & $>5$ HOURIWEEK & EFFORTIDIFFICULTY \\
\hline$>$ & $2-5$ HOURSIWEEK \\
\hline & 1-2 HOURSNWEK & A GREAT DEAL \\
& $<1$ HOURIWEEK & MUITE A LOT \\
\hline DID NOT DO & MODERATE \\
\hline
\end{tabular}

15. PROVIDING EMOTIONAL SUPPORT FOR OTHER CHILDREN IN THE FAMILY

\begin{tabular}{|c|c|}
\hline TIME & EFFORT/DIFFICULTY \\
\hline$>5$ HOURWEEK & - A GREAT DEAL \\
\hline$>2-5$ HOURS/WEEK & QUITE A LOT \\
\hline — 1-2 HOURSWEEK & MODERATE \\
\hline$<1$ HOURNEEK & A SMALL AMOUNT \\
\hline DID NOT DO & - NONE \\
\hline
\end{tabular}


16. PROVIDING EMOTIOINAL SUPPORT FOR THE EXTENDED FAMILY (EXAMPLES INCLUDE: GRANDPARENTS, AUNTS, UNCLES, FRIENDS, ETC.).

\begin{tabular}{|c|c|}
\hline \multicolumn{2}{|l|}{ TIME } \\
\hline & - $>5$ HOURNEEK \\
\hline & $>2-5$ HOURS WEEK \\
\hline & - 1-2 HOURSMEEK \\
\hline & - $<1$ HOURMEEK \\
\hline & DID NOT DO \\
\hline
\end{tabular}

EFFORT/DIFFICULTY
A GREAT DEAL
QUITE A LOT
MODERATE
A SMALL AMOUNT
NONE

17. PROVIDING EMOTIONAL SUPPORT FOR YOUR SPOUSEIPARTNER.

\begin{tabular}{ll} 
TIME & $>5$ HOURMEEK \\
\hline & $>2-5$ HOURSWWEEK \\
\hline & $1-2$ HOURS WEEK \\
& $<1$ HOURWEEK \\
\hline & DID NOT DO
\end{tabular}

\begin{tabular}{l} 
EFFORT/DIFFICULTY \\
A GREAT DEAL \\
\hline QUITE ALOT \\
MODERATE \\
A SMALLAMOUNT \\
NONE
\end{tabular}

18. MEETING YOUR OWN EMOTIONAL SUPPORT NEEDS.

\begin{tabular}{lll} 
TIME & $>5$ HOURNWEEK & EFFORTIDIFFICULTY \\
& $>2-5$ HOURS/WEEK & A GREAT DEAL \\
\hline & 1-2 HOURSWEEK & QUITE A LOT \\
& $<1$ HOURNWEK & MODERATE \\
\hline & DID NOT DO & A SMALL AMOUNT \\
\hline
\end{tabular}

19. COMFORTING YOUR CHILD THROUGH THE PAIN OF THE CANCER AND ITS TREATMENT (EXAMPLES INCLUDE: PROCEDURES, MOUTH SORES, BONE PAIN, ETC.).

\begin{tabular}{|c|c|}
\hline TIME & EFFORT/DIFFICULTY \\
\hline$>5$ HOURNEEK & A GREAT DEAL \\
\hline$>2-5$ HOURSIWEEK & - QUITE A LOT \\
\hline 1-2 HOURSNEEK & MODERATE \\
\hline$<1$ HOURWEEK & A SMALL AMOUNT \\
\hline _ DID NOT DO & NONE \\
\hline
\end{tabular}


20. TAKING CARE OF DISCIPLINE AND/OR BEHAVIOR PROBLEMS OF THE CHILD WITH CANCER (CRYING, IRRITABILITY, MOODINESS).

\begin{tabular}{|c|c|}
\hline \multicolumn{2}{|l|}{ TIME } \\
\hline & $>5$ HOURWEEK \\
\hline & $>2-5$ HOURS/WEEK \\
\hline & 1-2 HOURSNEEK \\
\hline & $<1$ HOURWEEK \\
\hline & DID NOT DO \\
\hline
\end{tabular}

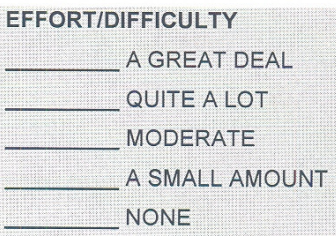

III. FINANCES

21. TAKING CARE OF FINANCES, BILLS, AND FORMS RELATED TO THE CHILD'S ILLNESS.

TIME

$>5$ HOURMEEK

$>2-5$ HOURS/WEEK

1-2 HOURSNEEK

$<1$ HOUR/WEEK

DID NOT DO
EFFORTIDIFFICULTY

A GREAT DEAL

QUITE A LOT

MODERATE

A SMALL AMOUNT

NONE

IV. FAMILYINTERPERSONAL RELATIONSHIPS

22. PLANNING ACTIVITIES FOR YOUR CHILD WITH CANCER AROUND THE TREATMENT AND ILLNESS (EXAMPLES INCLUDE: SCHOOL, PLAYTIME, REST, THINGS FOR THE CHILD TO DO, OTHER).

\begin{tabular}{ll}
$\frac{\text { TIME }}{\longrightarrow}$ & $>5$ HOURNWEK \\
& $>2-5$ HOURS/WEEK \\
& $1-2$ HOURSNWEK \\
& $<1$ HOURNEEK \\
\hline & DID NOT DO
\end{tabular}

\begin{tabular}{|c|c|}
\hline EFFORTIL & DIFFICULTY \\
\hline & A GREAT DEAL \\
\hline & QUITE A LOT \\
\hline & MODERATE \\
\hline & A SMALL AMOUNT \\
\hline & NONE \\
\hline
\end{tabular}

23. PLANNING ACTIVITIES FOR YOUR FAMILY AROUND THE TREATMENT AND ILLNESS (EXAMPLES INCLUDE: RECREATION, VACATION, SCHOOL FUNCTIONS, OTHER).

\begin{tabular}{lll} 
TIME & $>5$ HOURMEEK & EFFORTIDIFFICULTY \\
\hline & $>2-5$ HOURSWEEK & A GREAT DEAL \\
1-2 HOURSWEEK & QUITE A LOT \\
& $<1$ HOURNEEK & MODERATE \\
\hline DID NOT DO & A SMALL AMOUNT \\
\hline
\end{tabular}


24. GETTING CHILD CARE/BABYSITTING HELP FOR YOUR ILL CHILD.

\begin{tabular}{|c|c|}
\hline TIME & EFFORTIDIFFICULTY \\
\hline$>5$ HOURNEEK & A GREAT DEAL \\
\hline$\_>2-5$ HOURS $/$ WEEK & _ QUITE A LOT \\
\hline 1-2 HOURSMEEK & MODERATE \\
\hline$<1$ HOURNEEK & A SMALL AMOUNT \\
\hline DID NOT DO & _ NONE \\
\hline
\end{tabular}

25. OBTAINING CHILD CARE/BABYSITTING FOR BROTHERS AND SISTERS OF THE ILL CHILD.

\begin{tabular}{lll} 
TIME & $>5$ HOURMEEK & EFFORTIDIFFICULTY \\
\hline$>2-5$ HOURS WWEK & A GREAT DEAL \\
$1-2$ HOURSNWEK & QUITE A LOT \\
\hline & $<1$ HOURMEEK & MODERATE \\
\hline DID NOT DO & A SMALL AMOUNT \\
\hline
\end{tabular}

\section{COMMUNICATION}

26. COMMUNICATING INFORMATION ABOUT CANCER TO SCHOOLS, DAY CARE, BABYSITTERS, EXTENDED FAMILY AND FRIENDS.

\begin{tabular}{|c|c|}
\hline TIME & EFFORT/DIFFICULTY \\
\hline$\longrightarrow 5$ HOURMEEK & $\_$A GREAT DEAL \\
\hline _ $>2-5$ HOURSIWEEK & QUITE A LOT \\
\hline — 1-2 HOURSNEEK & MODERATE \\
\hline$<1$ HOURWEEK & A SMALL AMOUNT \\
\hline DID NOT DO & NONE \\
\hline
\end{tabular}

27. WATCHING FOR AND REPORTING YOUR CHILD'S PHYSICAL SYMPTOMS AND MEDICAL CONDITIOIN TO THE MEDICAL TEAM.

\begin{tabular}{lll} 
TIME & EFFORTIDIFFICULTY \\
\hline & $>5$ HOURNWEK & A GREAT DEAL \\
& $>2-5$ HOURSWWEK & QUITE A LOT \\
& $1-2$ HOURSNEEK & MODERATE \\
& $<1$ HOURMEEK & A SMALL AMOUNT \\
\hline DID NOT DO & NONE
\end{tabular}


28. GETTING INFORMATION ON YOUR CHILD'S ILLNESS AND THE TREATMENT (EXAMPLES INCLUDE: LIBRARY, MEDICAL TEAM, COMMUNITY AGENCIES).

\begin{tabular}{lll} 
TIME & $>5$ HOURMVEK & EFFORTIDIFFICULTY \\
& $>2$-5 HOURS WEEK & A GREAT DEAL \\
& 1-2 HOURSWWEEK & QUITE A LOT \\
& $<1$ HOURNWEEK & MODERATE \\
\hline DID NOT DO & A SMALL AMOUNT \\
\hline
\end{tabular}

29. IS THERE ANYTHING ELSE THAT YOU WISH TO TELL US ABOUT TAKING CARE OF YOUR CHILD WITH CANCER?

THANK YOU FOR HELPING US TO LEARN MORE ABOUT THE TIME AND EFFORT REQUIRED TO TAKE CARE OF A CHILD WHO IS RECEIVING TREATMENT FOR CANCER.

Reprint permission granted by Diane Keegan Wells, RN, MSN, CPNP, University of Maryland Medical Center, Baltimore, MD. 


\section{APPENDIX B}

REVISED INFORMAL CAREGIVERS SURVEY FOR PARENTS (RICS) 
Number

\section{Revised Informal Caregivers Survey (ICS) for Parents}

(Place an $X$ in the box that best represents you have felt over the past 5 days)

\begin{tabular}{|c|c|c|c|c|}
\hline Items & Very often & Often & Sometimes & Never \\
\hline \multicolumn{5}{|l|}{ 1. My child forgets things } \\
\hline \multicolumn{5}{|l|}{$\begin{array}{l}\text { 2. My child becomes } \\
\text { confused }\end{array}$} \\
\hline \multicolumn{5}{|l|}{$\begin{array}{l}\text { 3. My child refuses to } \\
\text { cooperate }\end{array}$} \\
\hline \multicolumn{5}{|l|}{ 4. My child becomes upset } \\
\hline \multicolumn{5}{|l|}{ 5. My child yells at me } \\
\hline \multicolumn{5}{|l|}{$\begin{array}{l}\text { 6. My child's behavior } \\
\text { embarrasses me }\end{array}$} \\
\hline \multicolumn{5}{|l|}{$\begin{array}{l}\text { 7. My child's behavior } \\
\text { embarrasses others }\end{array}$} \\
\hline \multicolumn{5}{|l|}{$\begin{array}{l}\text { 8. Taking care of my child } \\
\text { limits my free time }\end{array}$} \\
\hline \multicolumn{5}{|l|}{$\begin{array}{l}\text { 9. Taking care of my child } \\
\text { limits my social life }\end{array}$} \\
\hline \multicolumn{5}{|l|}{$\begin{array}{l}\text { 10. I don't have as much } \\
\text { privacy when I take care of } \\
\text { my child }\end{array}$} \\
\hline \multicolumn{5}{|l|}{$\begin{array}{l}\text { 11. I have to give my child } \\
\text { almost constant attention }\end{array}$} \\
\hline \multicolumn{5}{|l|}{$\begin{array}{l}\text { 12. Taking care of my child } \\
\text { has caused my health to get } \\
\text { worse }\end{array}$} \\
\hline \multicolumn{5}{|l|}{$\begin{array}{l}\text { 13. I have to take care of my } \\
\text { child when I don't feel well } \\
\text { enough }\end{array}$} \\
\hline \multicolumn{5}{|l|}{$\begin{array}{l}\text { 14. Taking care of my child } \\
\text { is hard on me emotionally }\end{array}$} \\
\hline \multicolumn{5}{|l|}{$\begin{array}{l}\text { 15. I do tasks my child could } \\
\text { be doing by himself/herself }\end{array}$} \\
\hline \multicolumn{5}{|l|}{$\begin{array}{l}\text { 16. I can't provide needed } \\
\text { medical care for my child }\end{array}$} \\
\hline $\begin{array}{l}17 \text { My sleep is interrupted } \\
\text { because I have to care for } \\
\text { my child }\end{array}$ & & & & \\
\hline
\end{tabular}

Source. Cafferta, G.L. \& Stone, R. (1989). The caregiving role: Dimensions of burden and benefits. Comprehensive Gerentology 2 Suppl., 57-64. Permission to modify granted by Joseph Ploanik, Webmaster, Center for Demographic Studies, Duke University Durham, NC. 
APPENDIX C

SLEEP DIARY 
SLEEP DIARY

Day 2 \& Day 5 before DEXAMETHASONE

Study ID Date

Instructions: Record about the previous day and night. Complete this sleep diary on day 3 about events on day 2 and on day 6 about events on day 5 before your child begins Dexamethasone.(See instructions for filling out sleep diary)

\begin{tabular}{|c|c|c|c|}
\hline \multicolumn{4}{|l|}{ Date } \\
\hline & Example & Day 2 & Day 5 \\
\hline $\begin{array}{l}\text { 1. I napped from __ to _ _ (note times of } \\
\text { all naps) }\end{array}$ & $2: 00$ to $2: 45$ & & \\
\hline $\begin{array}{l}\text { 2. I took mg of over-the- } \\
\text { counter/prescription medication and/or } \\
\text { oz of alcohol as a sleep aid. }\end{array}$ & Ambien $10 \mathrm{mg}$ & & \\
\hline 3. I smoked ___ \# of cigarettes & 2 & & \\
\hline $\begin{array}{l}\text { 4. I exercised (including vigorous house } \\
\text { cleaning for }\end{array}$ & $20 \mathrm{~min}$ & & \\
\hline $\begin{array}{l}\text { 5. I did the following activity and /or drank } \\
\text { and/or ate to help me sleep. }\end{array}$ & $\begin{array}{l}\text { Hot bath and } \\
\text { drank hot tea }\end{array}$ & & \\
\hline 6. I went to bed at _ o'clock. & $10: 30$ & & \\
\hline 7. I turned the lights out at ___o'clock. & $11: 15$ & & \\
\hline $\begin{array}{l}\text { 8. After turning the lights out I fell asleep in } \\
\text { minutes. }\end{array}$ & $10 \mathrm{~min}$ & & \\
\hline 9. My sleep was interrupted __ times & 2 & & \\
\hline $\begin{array}{l}\text { 10. My sleep was interrupted for } \\
\text { minutes. (specify duration of each } \\
\text { awakening) }\end{array}$ & $\begin{array}{l}30 \\
20\end{array}$ & & \\
\hline $\begin{array}{l}\text { 11. My sleep was interrupted by my child } \\
(0=\text { not at all, } 1,2,3,4=\text { a great deal }\end{array}$ & 1 & & \\
\hline $\begin{array}{l}\text { 12. I woke up at _o'clock (time of last } \\
\text { awakening) }\end{array}$ & $7: 15$ & & \\
\hline 13. I got out of bed at ___ o'clock & $7: 30$ & & \\
\hline $\begin{array}{l}\text { 14. When I got up this morning I felt } \\
\text { (1= exhausted } 1,2,3,4=\text { refreshed })\end{array}$ & 3 & & \\
\hline $\begin{array}{l}\text { 15. Overall my sleep last night was } \\
(1=\text { very restless, } 1,2,3,4=\text { very sound })\end{array}$ & 3 & & \\
\hline 16. I did not sleep sound because: & I had a cold. & & \\
\hline 17. My sleep was not good because: & $\begin{array}{l}\text { My child was } \\
\text { on IV meds }\end{array}$ & & \\
\hline
\end{tabular}




\section{SLEEP DIARY}

Day 2 \& Day 5 during DEXAMETHASONE

Study ID Date

Instructions: Record about the previous day and night. Complete this sleep diary on day 3 about events on day 2 and on day 6 about events on day 5 during your child's Dexamethasone. (See instructions for filling out sleep diary)

\begin{tabular}{|c|c|c|c|}
\hline Date & & & \\
\hline & Example & Day 2 & Day 5 \\
\hline $\begin{array}{l}\text { 1. I napped from } \\
\text { all naps) }\end{array}$ & $2: 00$ to $2: 45$ & & \\
\hline $\begin{array}{l}\text { 2. I took mg of over-the- } \\
\text { counter/prescription medication and/or } \\
\text { oz of alcohol as a sleep aid. }\end{array}$ & Ambien $10 \mathrm{mg}$ & & \\
\hline 3. I smoked ___ \# of cigarettes & 2 & & \\
\hline $\begin{array}{l}\text { 4. I exercised (including vigorous house } \\
\text { cleaning for } \quad \text { of minutes }\end{array}$ & $20 \mathrm{~min}$ & & \\
\hline $\begin{array}{l}\text { 5. I did the following activity and /or drank } \\
\text { and/or ate to help me sleep. }\end{array}$ & $\begin{array}{l}\text { Hot bath and } \\
\text { drank hot tea }\end{array}$ & & \\
\hline 6. I went to bed at _ _ o'clock. & $10: 30$ & & \\
\hline 7. I turned the lights out at ___o'clock. & $11: 15$ & & \\
\hline $\begin{array}{l}\text { 8. After turning the lights out I fell asleep in } \\
\text { minutes. }\end{array}$ & $10 \mathrm{~min}$ & & \\
\hline 9. My sleep was interrupted __ times & 2 & & \\
\hline $\begin{array}{l}\text { 10. My sleep was interrupted for } \\
\text { minutes. } \\
\text { (specify duration of each awakening) }\end{array}$ & $\begin{array}{l}30 \\
20\end{array}$ & & \\
\hline $\begin{array}{l}\text { 11. My sleep was interrupted by my child } \\
(0=\text { not at all, } 1,2,3,4=\text { a great deal }\end{array}$ & 1 & & \\
\hline $\begin{array}{l}\text { 12. I woke up at _o'clock (time of last } \\
\text { awakening) }\end{array}$ & $7: 15$ & & \\
\hline 13. I got out of bed at __ o'clock & $7: 30$ & & \\
\hline $\begin{array}{l}\text { 14. When I got up this morning I felt } \\
\text { (1= exhausted } 1,2,3, \underline{4=\text { refreshed })}\end{array}$ & 3 & & \\
\hline $\begin{array}{l}\text { 15. Overall my sleep last night was } \\
(1=\text { verv restless, } 1,2,3, \underline{4=\text { very sound })}\end{array}$ & 3 & & \\
\hline 16. My sleep was not good because: & $\begin{array}{l}\text { My child was on } \\
\text { IV meds during } \\
\text { the night. }\end{array}$ & & \\
\hline
\end{tabular}




\begin{tabular}{|l|l|l|l|}
\hline & during the night & & \\
\hline 18. My child slept with me: Yes or No & No & & \\
\hline $\begin{array}{l}\text { 19. Was this a typical night? If no please } \\
\text { explain. }\end{array}$ & Yes & & \\
\hline
\end{tabular}

20. Please answer the following questions thinking of the $\mathbf{1 0}$ days. Indicate the amount of caffeine you consume daily. Caffeine is found in coffee, tea cola drinks, Mountain Dew, chocolate, chocolate flavored drinks and anything else flavored with chocolate such as ice cream, cake, brownies, etc.

Number of beverages consumed containing caffeine

$\square \quad 1-2$ a day
$\square \quad 3-4$ a day
greater than 4
$\quad$ a day
$\square \quad$ None

Number of foods consumed containing caffeine

$\square \quad 1-2$ a day
$\square \quad 3-4$ a day
$\quad \quad \quad$ greater than 4
a day
$\quad$ None

Indicate the amount of alcohol you generally consume.

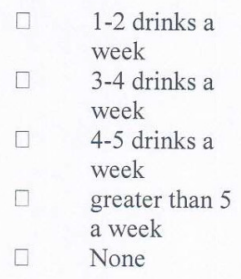

21. Identify medications you use now. Include prescribed medicines, vitamins, supplements, birth control pills, and any over-the-counter medicine.

Medication

Amount Taken

Time of day taken 
APPENDIX D

BRIEF FATIGUE INVENTORY (BFI) 


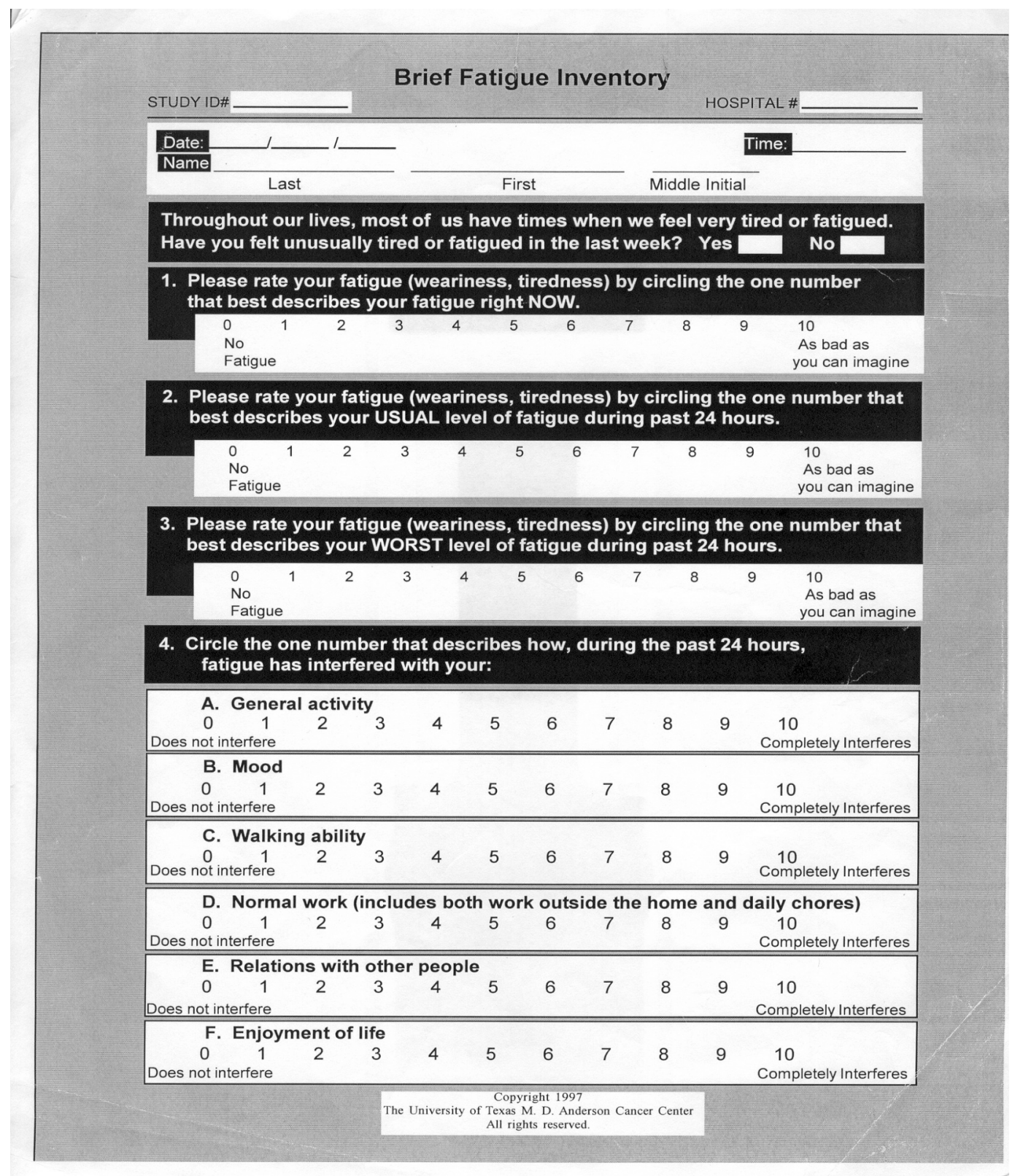

Reprint permission granted by Charles S. Cleeland, Ph.D., Pain Research Group. University of Texas M.D. Anderson, Houston, TX. 
APPENDIX E

PARENT DEMOGRAPHIC FORM 


$\begin{array}{cll} & \square \quad \text { Grandfather } \\ & \square \quad \text { Other } \\ \text { Employment } & \square \quad \begin{array}{l}\text { Officials and Managers } \\ \text { Professional and Related Occupations } \\ \square \quad\end{array} \\ \square \quad \text { Technologist and Technicians } \\ \square \quad \text { Sales and Related Occupations } \\ \square \quad \text { Construction and Maintenance and Repair } \\ \square \quad \text { Production and Transportation } \\ \square \quad \text { Laborers, Helpers and Material Handlers } \\ \square \quad \text { Homemakers } \\ \square \quad \text { Health Professional } \\ \square \quad \text { Farmers } \\ \square \quad \text { Retired } \\ \square \quad \text { Unemployed } \\ \square \quad \text { Student } \\ \square \quad \text { Self-Employed }\end{array}$

Are you currently employed?

$\square \quad$ Yes

$\square \quad$ No

Have you continued to work since your $\quad \square \quad$ Yes child was diagnosed with cancer? $\quad \square \quad$ No

If no, would you have continued working if $\square \quad$ Yes you were not taking care of your child? $\quad \square \quad$ No

If yes, then do you work shifts? $\quad \square \quad$ Yes

What shift do you work? $\quad \square \quad$ On call

$\square \quad$ Days

$\square \quad$ Evening

Nights

How many hours a week do you work? $\quad \square \quad$ 1-12 hours

13-24 hours

25-36 hours

$37-40$ hours

Greater than 40 hours

Are you working fewer hours a week

Yes

because you are caring for your child? $\quad \square \quad$ No 


\begin{tabular}{|c|c|c|}
\hline Education & $\begin{array}{l}\square \\
\square \\
\square \\
\square \\
\square \\
\square\end{array}$ & $\begin{array}{l}\text { Less that High School } \\
\text { High School Graduate } \\
\text { Some College } \\
\text { College Graduate } \\
\text { Post College Graduate } \\
\text { Vo-Tech or Trade School }\end{array}$ \\
\hline Usual Level of Activity & $\begin{array}{l}\square \\
\square \\
\square\end{array}$ & $\begin{array}{l}\text { High } \\
\text { Moderate } \\
\text { Low }\end{array}$ \\
\hline Do you usually exercise? & $\begin{array}{l}\square \\
\square\end{array}$ & $\begin{array}{l}\text { Yes } \\
\text { No }\end{array}$ \\
\hline If yes, type of exercise? & $\begin{array}{l}\square \\
\square \\
\square \\
\square \\
\square \\
\square\end{array}$ & $\begin{array}{l}\text { Running/ Jogging } \\
\text { Aerobics } \\
\text { Swimming } \\
\text { Bicycling } \\
\text { Weight Training } \\
\text { Other }\end{array}$ \\
\hline How many times per week? & $\begin{array}{l}\square \\
\square \\
\square\end{array}$ & $\begin{array}{l}\text { 1-2 times a week } \\
3-4 \text { times a week } \\
4 \text { or more times a week }\end{array}$ \\
\hline How long do you exercise? & $\begin{array}{l}\square \\
\square \\
\square\end{array}$ & $\begin{array}{l}15-30 \text { minutes } \\
30 \text { minutes- } 1 \text { hour } \\
\text { Greater than } 1 \text { hour }\end{array}$ \\
\hline House Hold Income & $\begin{array}{l}\square \\
\square \\
\square\end{array}$ & $\begin{array}{l}\text { Less than } \$ 20,000 \\
20,000 \text { to } \$ 40,000 \\
\text { Over } \$ 40,000\end{array}$ \\
\hline Residence & $\begin{array}{l}\square \\
\square \\
\square \\
\square\end{array}$ & $\begin{array}{l}\text { Own Home } \\
\text { Rent Home } \\
\text { Rent Apartment } \\
\text { Other }\end{array}$ \\
\hline Tennessee Resident & $\begin{array}{l}\square \\
\square\end{array}$ & $\begin{array}{l}\text { Yes } \\
\text { No }\end{array}$ \\
\hline $\begin{array}{l}\text { Health } \\
\text { Are you in good health? }\end{array}$ & $\square$ & Yes \\
\hline
\end{tabular}


If no, please describe your health problems.

\section{Sleep Patterns}

According to your usual routine, what time do you generally:

Wake up

Go to Sleep

Do you have any sleeping problems?

Does your child sleep in the bed with you? $\quad \square \quad$ No

If yes, please describe your sleeping problems?

Indicate the amount of caffeine you consume daily. Caffeine is found in coffee, tea cola drinks, Mountain Dew, chocolate, chocolate flavored drinks and anything else flavored with chocolate such as ice cream, cake, brownies, etc.

Number of beverages consumed containing caffeine

Indicate the amount of alcohol you generally consume.
1-2 a day

3-4 a day

greater than 4 a day

$1-2$ a day

3-4 a day

greater than 4 a day

1-2 drinks a week

3-4 drinks a week

$4-5$ drinks a week 
If no, please describe your health problems.

\section{Sleep Patterns}

According to your usual routine, what time do you generally:

Wake up

Go to Sleep

Do you have any sleeping problems?

Does your child sleep in the bed with you? $\quad \square \quad$ No

If yes, please describe your sleeping problems?

Indicate the amount of caffeine you consume daily. Caffeine is found in coffee, tea cola drinks, Mountain Dew, chocolate, chocolate flavored drinks and anything else flavored with chocolate such as ice cream, cake, brownies, etc.

Number of beverages consumed containing caffeine

Indicate the amount of alcohol you generally consume.
1-2 a day

3-4 a day

greater than 4 a day

$1-2$ a day

3-4 a day

greater than 4 a day

1-2 drinks a week

3-4 drinks a week

$4-5$ drinks a week 
Greater than 5 a week none

Identify medications you use now. Include prescribed medicines, vitamins, supplements, birth control pills, and any over-the-counter medicine.
Medication
Amount Taken
Time of day taken

Please indicate if you would like to be reminded of collection times or complete forms via telephone $\quad \square \quad$ Yes

Comments:

Thank-you! 
APPENDIX F

FEASIBILITY STUDY LETTER 
APPENDIX G

FEASIBILITY STUDY DEMOGRAPHIC FORM 
Were the directions for the Informal Caregiver Survey easy to follow?

Yes or No

Did you find that the format of the Informal Caregiver Survey was easy to use?

Yes or No

Is there an additional item that you feel should be included on the Informal Caregiver Survey Revised for parents?

Yes or No

Comments:

Using the following page, please read each item and circle the number on this sheet that most accurately reflects how easy it was to understand each item.

$1=$ The item was not easy to understand

$2=I$ can understand the item but it needs minor revisions to make it clearer

$3=$ The item was easy to understand 
APPENDIX H

FEASIBILITY RELEVANT FORM 


\section{Feasibility Relevant Form}

Using the following page, please read each item and circle the number on this sheet that most accurately reflects how you feel each item represents "subjective burden: for parents with regard to caring for your child who is ill. Subjective Burden is defined as feelings, emotions and attitudes related to the caregiving experience.

$1=$ The item was not relevant

$2=\mathrm{I}$ was unable to determine relevancy

$3=$ The item was relevant, but it needs minor revisions to make it more

relevant

4= The item was very Relevant 


\section{RELEVANT}

\begin{tabular}{|l|c|c|c|c|}
\hline Items & $\begin{array}{c}\text { Not } \\
\text { Relevant }\end{array}$ & $\begin{array}{c}\text { Unable to } \\
\text { Assess }\end{array}$ & $\begin{array}{c}\text { Relevant with } \\
\text { Revisions }\end{array}$ & $\begin{array}{c}\text { Very } \\
\text { Relevant }\end{array}$ \\
\hline 1. My child forgets things & 1 & 2 & 3 & 4 \\
\hline 2. My child becomes confused & 1 & 2 & 3 & 4 \\
\hline 3. My child refuses to cooperate & 1 & 2 & 3 & 4 \\
\hline 4. My child becomes upset & 1 & 2 & 3 & 4 \\
\hline 5. My child yells at me & 1 & 2 & 3 & 4 \\
\hline 6. My child's behavior embarrasses me & 1 & 2 & 3 & 4 \\
\hline 7. My child's behavior embarrasses others & 1 & 2 & 3 & 4 \\
\hline $\begin{array}{l}\text { 8. Taking care of my child limits my free time } \\
\text { 9. Taking care of my child limits my social life }\end{array}$ & 1 & 2 & 3 & 4 \\
\hline $\begin{array}{l}\text { 10. I don't have as much privacy because I } \\
\text { care of my child }\end{array}$ & 1 & 2 & 3 & 4 \\
\hline $\begin{array}{l}\text { 11. I have to give my child almost constant } \\
\text { attention }\end{array}$ & 1 & 2 & 3 & 4 \\
\hline $\begin{array}{l}\text { 12. Taking care of my child has caused my } \\
\text { health to get worse }\end{array}$ & 1 & 2 & 3 & 4 \\
\hline $\begin{array}{l}\text { 13. I have to take care of my child when I } \\
\text { don't feel well enough }\end{array}$ & 1 & 2 & 3 & 4 \\
\hline $\begin{array}{l}\text { 14. Taking care of my child is hard on me } \\
\text { emotionally }\end{array}$ & 1 & 2 & 3 & 4 \\
\hline $\begin{array}{l}\text { 15. I do tasks my child could be doing by } \\
\text { himself/herself }\end{array}$ & 1 & 2 & 3 & 4 \\
\hline $\begin{array}{l}\text { 16. I can't provide needed medical care for } \\
\text { my child }\end{array}$ & 1 & 2 & 3 & 3 \\
\hline $\begin{array}{l}\text { 17. My sleep is interrupted because I have to } \\
\text { care for my child }\end{array}$ & 1 & 2 & & 4 \\
\hline
\end{tabular}


Were the directions for the Informal Caregiver Survey easy to follow?

Yes or No

Did you find that the format of the Informal Caregiver Survey was easy to use?

Yes or No

Is there an additional item that you feel should be included on the Informal Caregiver Survey Revised for parents?

Yes or No

Comments: 
APPENDIX I

FEASIBILITY EASY TO UNDERSTAND FORM 


\section{Feasibility Easy to Understand Form}

Using the following page, please read each item and circle the number on this sheet that most accurately reflects how easy it was to understand each item.

$\mathbf{1}=$ The item was not easy to understand

$\mathbf{2}=\mathrm{I}$ can understand the item but it needs minor revisions to make it clearer

$\mathbf{3}=$ The item was easy to understand 


\section{EASY TO UNDERSTAND}

\begin{tabular}{|c|c|c|c|}
\hline Items & $\begin{array}{l}\text { Not Easy to } \\
\text { Understand }\end{array}$ & $\begin{array}{c}\text { Easy to } \\
\text { Understand with } \\
\text { Revisions } \\
\end{array}$ & $\begin{array}{l}\text { Very Easy to } \\
\text { Understand }\end{array}$ \\
\hline 1. My child forgets things & 1 & 2 & 3 \\
\hline $\begin{array}{l}\text { 2. My child becomes } \\
\text { confused }\end{array}$ & 1 & 2 & 3 \\
\hline $\begin{array}{l}\text { 3. My child refuses to } \\
\text { cooperate }\end{array}$ & 1 & 2 & 3 \\
\hline 4. My child becomes upset & 1 & 2 & 3 \\
\hline 5. My child yells at me & 1 & 2 & 3 \\
\hline $\begin{array}{l}\text { 6. My child's behavior } \\
\text { embarrasses me }\end{array}$ & 1 & 2 & 3 \\
\hline $\begin{array}{l}\text { 7. My child's behavior } \\
\text { embarrasses others }\end{array}$ & 1 & 2 & 3 \\
\hline $\begin{array}{l}\text { 8. Taking care of my child } \\
\text { limits my free time }\end{array}$ & 1 & 2 & 3 \\
\hline $\begin{array}{l}\text { 9. Taking care of my child } \\
\text { limits my social life }\end{array}$ & 1 & 2 & 3 \\
\hline $\begin{array}{l}\text { 10. I don't have as much } \\
\text { privacy when I take care of } \\
\text { my child }\end{array}$ & 1 & 2 & 3 \\
\hline $\begin{array}{l}\text { 11. I have to give my child } \\
\text { almost constant attention }\end{array}$ & 1 & 2 & 3 \\
\hline $\begin{array}{l}\text { 12. Taking care of my child } \\
\text { has caused my health to get } \\
\text { worse }\end{array}$ & 1 & 2 & 3 \\
\hline $\begin{array}{l}\text { 13. I have to take care of my } \\
\text { child when I don't feel well } \\
\text { enough }\end{array}$ & 1 & 2 & 3 \\
\hline $\begin{array}{l}\text { 14. Taking care of my child } \\
\text { is hard on me emotionally }\end{array}$ & 1 & 2 & 3 \\
\hline $\begin{array}{l}\text { 15. I do tasks my child could } \\
\text { be doing by himself/herself }\end{array}$ & 1 & 2 & 3 \\
\hline $\begin{array}{l}\text { 16. I can't provide needed } \\
\text { medical care for my child }\end{array}$ & 1 & 2 & 3 \\
\hline $\begin{array}{l}\text { 17. My sleep is interrupted } \\
\text { because I have to care for } \\
\text { my child }\end{array}$ & 1 & 2 & 3 \\
\hline
\end{tabular}


APPENDIX J

INSTRUCTIONS FOR SLEEP DIARY 

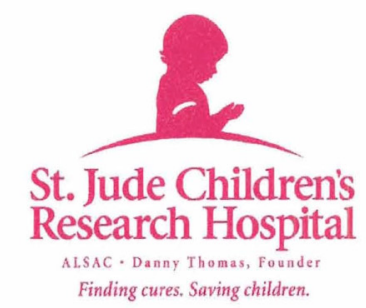

Date

Dear [actual name of parent]

We are writing to parents of children, who are currently enrolled on Total XV in continuation therapy approaching or past week 50, asking them to consider participating in a new research study. We will describe the study in this letter and we welcome any questions you may have.

As a parent of a child with ALL, we realize that you are very involved in the day-to-day care of your child. We want to be as helpful to you as possible because we believe that proper attention to the needs of the parents is essential to the care and health outcome of the patient. We want to learn more about the symptoms that you experience during the time that your child is taking dexamethasone. Parents often report that their child experiences altered sleep patterns, fatigue, irritability and other behavioral changes. We want to know if these types of changes in your child's behavior have any affect on your sleep pattern, fatigue, and caregiving duties. We believe that the best ways to learn about these symptoms is to invite parents to share your experience with us by completing several questionnaires regarding these symptoms and by wearing a wrist actigraph (a watch-like instrument used to measure sleep and activity) before and during the time your child is on dexamethasone.

Enclosed you will find forms and instructions for this study. We are happy that you have decided to participate in this study. Please know that you may change your mind at any time and that your responses to the questionnaires will be kept confidential. If you so desire we will be glad to contact you via telephone to remind you of data collection times or to complete your forms over the phone

Please feel free to contact either of us at the hospital with any questions about this study at (901) 495-3679. We would be very glad to answer any questions that you may have regarding this study. You will receive a $\$ 25.00$ meal certificate as compensation for your participation in this study.

Sincerely,

Pam Hinds, PhD, RN CS

Jerithea Tidwell, MSN, RN, CNS 


\section{INSTRUCTIONS FOR FILLING OUT SLEEP DAIRY}

To better understand sleep efficiency of parents with children who are on Dexamethasone for ALL therapy, we would like to collect some important information on your sleep pattern. After you get up in the morning please answer all 12 questions on the sleep diary. It is important that you complete this diary every morning. For example, when you get up on day 3 before your child's dexamethasone is scheduled to begin, complete the column under Day 2 about the day before. When you get up on day 1 that your child's dexamethasone is scheduled to begin complete the column under day 5 (before dexamethasone) about the day before. It is difficult to estimate how long you take to fall asleep or how long you are awake at night. However, we want only your best estimates. If some unusual events occurs on a given night (E.g. illness, emergency, phone call) make a note of it. Below are the guidelines to help you answer each question. An example is also provided on the diary.

1. Napping: This should include all naps even though they were not intentional. For instance, $f$ you dozed off in front of the TV for 10 minutes, please write this down. Make sure to specify a.m. or p.m.

2. Sleep Medication: Please include both prescribed and over-the-counter medications as ell as alcohol used specifically for a sleep aid including supplements and vitamins.

3. Exercise: Write down the amount of time you spent exercising in minutes

4. Sleep Aids: Write any activity, food or drink that you may have done or consumed to help you sleep.

5. Bedtime: This is the time you go to bed. If you go to bed at 10:45 but turn the lights off at 11:15, you should write 10:45 in the "I went to bed at block".

6. Lights Off: This is the time you go to bed and actually turn the lights off. If you go to bed at 10:45 but turn the lights off at 11:15, you should write 11:15 in the "I turned the lights out at block.

7. Sleep-Onset Latency: Provide the best estimate of how long it took you to fall asleep after you turned the lights off ants intended to fall to sleep.

8. Number of Awakenings: This is the number of times you remember waking up during the night.

9. Duration of Awakenings: Please estimate to the best of your knowledge, how many minutes you spent awake for each awakening. If this is impossible then estimate the number of minutes you spent awake for all awakenings combined.

10. Sleep Interruption by My Child: Please indicate if your sleep was interrupted by your child with cancer during the night.

11. Morning awakening: This is the last time you awaken in the morning. If you awoke at 4:00 and never went back to sleep this is the time to write. However, if you wake at 4:00 but went back to sleep for a brief period of time (for example from 6:00 to 6:20) then your last awakening would be $6: 20$.

12. Out of Bed Time: This is the time you actually got out of bed for the day.

13. Feeling upon Rising: $1=$ Exhausted, $2=$ Tired, $3=$ Average, $4=$ Refreshed).

14. Sleep Quality: $1=$ Very restless, $2=$ Restless, $3=$ Average quality $4=$ Sound)

15. Reason for Overall Sleep: If you indicated a 1 or 2 to describe your overall sleep please describe reason sleep was not sound. 
APPENDIX K

SAMPLE PARENT PARTICIPANT LETTER 


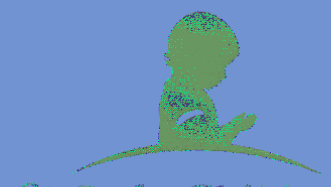

St Jude Children's

Research Hospital

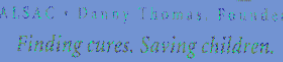

Date

Dear Research Participant

We are writing to parents of children, who are currently enrolled on Total $X V$ in continuation therapy approaching or past week 50 , asking them to consider participating in a new research study. We will describe the study in this lever and we welcome any questions you may have.

As a parent of a child with ALi.. we realize that you are very involved in the day -today care of your child. We want to be as helpful to you as possible because we believe that proper attention to the needs of the parents is essential to the care and heal outcome of the patient. We want to lean more about the symptoms that you experience during the time that your child is taking dexamethasone. Parents often report that their child experiences altered sleep patters, fatigue, initability and other behavioral changes. We want to know if these types of changes in your child's behavior have any affect on your sleep patter, fatigue, and caregiving duties. We believe that the best ways to lean about these symptoms is to invite parents to shat your experience with us by completing several questionnaires regarding these symptoms and by wearing a wrist actigraph (a watch-like instrument used to measure sleep and activity) before and during the time jour child is on dexamethasone.

Enclosed you will find forms and instructions for this study. We are happy that you have decided to participate in this study. Please know that you may change your mind at any time and that your responses to the questionnaires will be kept confidential. If you so desire we will be glad to contact you via telephone to remind you of data collection times or to complete your. forms over the phone

Please feel free to contact either of us at the hospital with any questions about this study at (901) 495-3679. We would be very glad to answer any questions that you may have regarding this study. You will receive a $\$ 25,00$ meal certificate as compensation for your participation in this study.

Sincerely,

Pan Hinds, PhD, RY CS

Jerithea Tidwei!, MSN, RN, CNS

141 
APPENDIX L

INSTRUCTIONS FOR COMPLETING WRITTEN FORMS 


\section{INSTRUCTIONS FOR COMPLETING WRITTEN FORMS}

All of the yellow forms are to be filled out before your child's Dexamethasone therapy begins. The purple forms are to be filled out during your child's Dexamethasone therapy.

1. Complete the blue demographic form.

2. Complete Brief Fatigue Inventory on Day $\mathbf{5}$ before your child begins Dexamethasone and on Day $\mathbf{5}$ during your child' Dexamethasone therapy. Fatigue is defined as a feeling of physical and or mental weariness or tiredness resulting from exertion or stress. Please complete this form thinking of your experiences and feelings during the past week. Please complete all the items on the Brief Fatigue Inventory

3. Complete the Care of My Child with Cancer Instrument on Day $\mathbf{5}$ before your child's Dexamethasone begins and then again on Day $\mathbf{5}$ during your child's Dexamethasone therapy thinking about the last week. This instrument will be used to learn more about the time, tasks, and effort that is needed in caring for a child with cancer. Please complete all the items on this instrument.

4. Complete the Revised informal caregiver's Survey for Parents on Day $\mathbf{5}$ before your child's Dexamethasone begins and then again on Day $\mathbf{5}$ during your child's Dexamethasone therapy thinking about the last week. This survey will be used to learn more about subjective burden. Subjective burden is the defined as feelings, emotions and attitudes related to the caregiving experience. Make sure that you complete all the items on the Survey.

5. Complete the Sleep Diary on day 2 and 5 before Dexamethasone therapy and on days 2 and 5 during Dexamethasone therapy. Instructions are found on the INSTRUCTIONS FOR FILLING OUT SLEEP DIARY. Please answer all the questions about your sleep during the night on the next morning.

6. Wear the Wrist Actigraph on your non-dominant arm at all times for 10-days and nights. DO NOT GET THE WATCH WET! You should take it off when you shower or take a bath, work in water that may splash on you or go swimming. Avoid very humid conditions such as the bathroom (leave it on your dresser during a shower or bath). Please where the Actigraph at all times except during shower or bath.

If you have any questions please call:

Jerithea Tidwell RN MSN 495-5462; pager 4953578 enter 2473, 372-2940 (H) 


\section{APPENDIX M}

DATA COLLECTION TIME LINE 


\begin{tabular}{|c|c|c|c|c|c|c|c|c|c|c|}
\hline Instruments & $\begin{array}{l}\text { Day } \\
1\end{array}$ & $\begin{array}{l}\text { Day } \\
2\end{array}$ & \begin{tabular}{|l|} 
Day \\
\end{tabular} & $\begin{array}{l}\text { Day } \\
4\end{array}$ & $\begin{array}{l}\text { Day } \\
5\end{array}$ & $\begin{array}{l}\text { Day } \\
1\end{array}$ & $\begin{array}{l}\text { Day } \\
2\end{array}$ & \begin{tabular}{|l|} 
Day \\
\end{tabular} & $\begin{array}{l}\text { Day } \\
4\end{array}$ & $\begin{array}{l}\text { Day } \\
5\end{array}$ \\
\hline $\begin{array}{l}\text { Demographic } \\
\text { Form }\end{array}$ & $\sqrt{ }$ & & & & & & & & & \\
\hline $\begin{array}{l}\text { Brief Fatigue } \\
\text { Inventory }\end{array}$ & & $\sqrt{ }$ & & & $\sqrt{ }$ & & $\sqrt{ }$ & & & $\sqrt{ }$ \\
\hline Sleep Diary & & $\sqrt{ }$ & & & $\sqrt{ }$ & & $\sqrt{ }$ & & & $\sqrt{ }$ \\
\hline Actigraph & $\sqrt{ }$ & $\sqrt{ }$ & $\sqrt{ }$ & $\sqrt{ }$ & $\sqrt{ }$ & $\sqrt{ }$ & $\sqrt{ }$ & $\sqrt{ }$ & $\sqrt{ }$ & $\sqrt{ }$ \\
\hline $\begin{array}{l}\text { Care of My } \\
\text { child with } \\
\text { Cancer } \\
\text { Instrument }\end{array}$ & & & & & $\sqrt{ }$ & & & & & $\sqrt{ }$ \\
\hline $\begin{array}{l}\text { Revised ICS } \\
\text { for Parents }\end{array}$ & & & & & $\sqrt{ }$ & & & & & $\sqrt{ }$ \\
\hline
\end{tabular}


APPENDIX N

INFORMED CONSENT STATEMENT 


\section{SLEEP, FATIGUE, AND CAREGIVER BURDEN IN PARENTS OF CHILDREN WITH ACUTE LYMPHOBLASTIC LEUKEMIA (ALL)}

Caring for a child with cancer can be physically and mentally challenging. The health status of a parent can be influenced by the health of the child. Parents often report that their child diagnosed with ALL and receiving steroids, experience symptoms such as altered quality of sleep, irritability, and decreased cooperation with health procedures such as taking medications. Therefore, care of a child with these symptoms may disrupt parents' sleep and may also contribute to an increase in fatigue. Giving care to a child with ALL who is taking dexamethasone can be more difficult if the parents are tired and sleep deprived. Although these are common complaints, altered sleep quality, fatigue, and caregiver burden have not been studied in parents during their child's treatment with dexamethasone.

In this study we are interested in discovering how parent's sleep and their level of fatigue are affected during the time five days before and during their child with ALL treatment with dexamethasone. We also want to learn if these symptoms have any influence on care giving practices. As healthcare providers, we want to better understand these events so that we can decrease fatigue and develop a plan to help parents cope so that they can continue to provide appropriate care to their child during this treatment period.

We are inviting you to participate in this research study. This consent form gives you information about the study, which will be discussed with you in order to help you make an informed decision on whether or not to participate. If you have any questions, do not hesitate to ask. Once you understand the study, and if you agree to participate, you will be asked to sign this consent form. You will be given a copy of the consent form to keep.

Before you learn about the study, it is important that you know the following:

- Whether or not you take part in this study is entirely up to you.

- If you decide not to participate in the study, or to withdraw from the study at any time your child will not lose the benefits to routine medical care.

- $\quad$ You may choose to participate in other studies regarding fatigue, sleep, and caregiver burden, if available.

- This study is being sponsored by National Institute of Nursing Research, National Institute of Health (NINR, NIH)

\section{Why is this study being done?}

The purpose of this study is to measure parents' sleep quality, level of fatigue, and caregiver burden 5 days before the child begins dexamethasone and then again for 5 days during the time the child is on dexamethasone.

The study has the following goals: 
1. To explore whether there is a change in parental sleep quality during two consecutive 5-day periods (when their child is off versus on dexamethasone).

2. To explore whether there is a difference in parent's level of fatigue during two consecutive 5-day periods (when their child is off versus on dexamethasone).

3. To learn about the parent's caregiver burden (time, effort, strain, and difficulty of care responsibilities) during the two consecutive 5-day periods (when their child is off versus on dexamethasone).

4. To learn if there is any relationship among parent sleep quality, fatigue, and caregiver burden within each of the 5-day periods (when their child is off versus on dexamethasone).

5. To learn about the relationships among parents' sleep efficiency, sleep duration, fatigue and caregiver burden within each of the 5-day periods

6. To compare the sleep quality and level of fatigue of parents with that of their child who is receiving dexamethasone for treatment of ALL during the two consecutive 5-day periods (off versus on dexamethasone).

\section{How many patients will take part in the study?}

We hope that about 39 parents or guardians of children treated at St. Jude Children's Research Hospital will take part in this study.

\section{What is involved in this study?}

You will be given a study packet. The study packet includes an information letter, an instruction sheet, which contains detail instructions on completing the surveys, the Brief Fatigue Surveys, Sleep Diary Forms, the Care of My Child with Cancer Instrument, the Revised Informal Caregiver's Survey for Parents, and a Parent Demographic Form. You will also get a wrist actigraph to wear on your non-dominant hand for the 10 days of this study. A wrist actigraph is similar to a watch. It has a computer chip in it. The computer chip will keep track of the way you move when you are awake or asleep. The wristband is light and it can be taken off if necessary (for example, if you prefer not to get the wristband wet, you may remove the actigraph when bathing, swimming, or showering).

You will be asked to complete different questionnaires. It will take about 25 minutes of your time per week to complete them. The following table shows a timeline of when you will fill out the questionnaires.

With your permission, we will contact you via telephone to remind you of data collection time points. Please initial and date your choice at the end of this consent form. 


\begin{tabular}{|c|c|c|c|c|c|c|c|c|c|c|c|}
\hline Instruments & $\begin{array}{l}\text { Study } \\
\text { enrollment }\end{array}$ & $\begin{array}{l}\text { Day } \\
1\end{array}$ & \begin{tabular}{|l} 
Day \\
2
\end{tabular} & $\begin{array}{l}\text { Day } \\
3\end{array}$ & $\begin{array}{l}\text { Day } \\
4\end{array}$ & $\begin{array}{l}\text { Day } \\
5\end{array}$ & $\begin{array}{l}\text { Day } \\
1\end{array}$ & $\begin{array}{l}\text { Day } \\
2\end{array}$ & $\begin{array}{l}\text { Day } \\
3\end{array}$ & $\begin{array}{l}\text { Day } \\
4\end{array}$ & $\begin{array}{l}\text { Day } \\
5\end{array}$ \\
\hline $\begin{array}{l}\text { Parent } \\
\text { Demographic } \\
\text { Form }\end{array}$ & $\sqrt{ }$ & & & & & & & & & & \\
\hline $\begin{array}{l}\text { Brief Fatigue } \\
\text { Inventory }\end{array}$ & & & $\sqrt{ }$ & & & $\sqrt{ }$ & & $\sqrt{ }$ & & & $\sqrt{ }$ \\
\hline Sleep Diary & & & $\sqrt{ }$ & & & $\sqrt{ }$ & & $\sqrt{ }$ & & & $\sqrt{ }$ \\
\hline Actigraph & & $\sqrt{ }$ & $\sqrt{ }$ & $\sqrt{ }$ & $\sqrt{ }$ & $\sqrt{ }$ & $\sqrt{ }$ & $\sqrt{ }$ & $\sqrt{ }$ & $\sqrt{ }$ & $\sqrt{ }$ \\
\hline $\begin{array}{l}\text { Care of My } \\
\text { child with } \\
\text { Cancer } \\
\text { Instrument }\end{array}$ & & & & & & $\sqrt{ }$ & & & & & $\sqrt{ }$ \\
\hline $\begin{array}{l}\text { Revised ICS } \\
\text { for Parents }\end{array}$ & & & & & & $\sqrt{ }$ & & & & & $\sqrt{ }$ \\
\hline
\end{tabular}

\section{How long will I be in the study?}

You will be receiving a study form packet at the beginning of the study. You will answer the questions as so stated in the instructions on days 2 and 5 before your child begins his or her dexamethasone treatment and then again on days 2 and 5 during your child's dexamethasone treatment. After this 10-day period you will return your wrist actigraph and completed parent packet. You will be provided with self-addressed envelope to mail back the completed forms. You will also be given a box with a pre-paid Fed-Ex form addressed to St Jude for returning the wrist actigraph. This will end your participation in this study.

\section{Can I be taken out of the study without my consent?}

You may be taken out of the study without your consent for the following reasons:

- New information is learned that a better study/intervention/observation is available, or that the study is not in your best interest.

- The study sponsor decides to end the study.

\section{What are the consequences of withdrawing from this study?}

You can choose to stop the study at any time. There are no consequences for withdrawing from the study. 


\section{What are the risks of the study?}

We believe that the risks of the study are very small. One possible risk may be that some of the questions about caregiver burden may make you feel uncomfortable. The word "burden" can cause a negative or guilt-like feeling. It is not our intention to make you feel uncomfortable or to make you feel as though you are complaining about the care you have to give to your child with cancer. Another possible risk or discomfort that you could experience during this study includes discomfort from wearing the actigraph and fatigue when completing the questionnaires. It is expected that you will get used to the actigraph quickly. Every attempt will be made to help make participating in this study easy for you.

\section{What are the benefits of the study?}

Taking part in this study may not benefit you. However, the information we learn from this study may help other parents/guardians and patients in the future.

\section{What other options are there?}

You can choose not to take part. If you choose not to take part, your child will still receive the normal care given to all patients.

\section{What about new information?}

You will be told of any new information learned during the course of the study, which might cause you to change your mind about staying in the study. You have the right to learn about the results of the study. If you are interested in learning more about when and how to get the results of this research study, you may contact Dr. Pam Hinds at 901/4953679 .

\section{What about confidentiality?}

No information other than what is needed for the study will be recorded. Every effort is made to protect your privacy. Your answers and other study data will be kept in locked files in the Nursing Research department at St. Jude. This information will not be shared with anyone outside of the study staff. The information we get from you will be entered into the computer with a research participant study number only. Any reports or publications that result from this data will have no information that could be used to identify study subjects.

Government agencies oversee research studies involving people. Your research records may be reviewed by such agencies if you take part in this research study. These agencies include the Food and Drug Administration (FDA), the National Cancer Institute (NCI), the National Institute of Nursing Research (NINR), an oversight committee from within St. Jude By signing this consent form, you are allowing your research records to be reviewed by these persons. 


\section{SUMMARY OF RESEARCH AND \\ PRIVACY RIGHTS \\ Non-Therapeutic Research}

IRB version: November 4, 2003

\section{I have been told the following information:}

1) I may talk as much as I want to with Dr. Hinds about the reasons for this study and about its risks.

2) This study may have risks that the researchers do not know about now.

3) There will be no additional costs to me from taking part in this research study. It is the policy of St. Jude Children's Research Hospital not to bill research participants or their families for the cost of medical care. This includes any costs associated with taking part in research studies.

4) I/my family will receive a $\$ 25.00$ certificate from McDonald's or Wal-Mart as compensation for being in this study.

5) The hospital policy is not to provide payment if I am injured or damaged by being in this study. I have been told that St. Jude will treat any such injury only to the extent the hospital decides is reasonably possible, using the staff, supplies, and physical facilities of the hospital. When treatment is necessary beyond what St. Jude can provide, St. Jude will help me locate suitable medical care elsewhere.

6) If I choose not to enroll on this research study, I may still be eligible to participate in other research studies at St. Jude Children's Research Hospital. I have been told that I can withdraw from this study at any time. If this happens, my child may still be treated at St. Jude Children's Research Hospital.

7) I received a copy of the St. Jude Notice of Privacy Practices at the time of registration. That document tells me how my medical information may be used or disclosed (given to someone outside the hospital). I have been told I have the right to review the Notice of Privacy Practices before I sign this form. I have been told that I may request another copy of the Notice of Privacy Practices and that it is posted in the Hospital and on our website (www.stjude.org).

8) I have the right to inspect, copy and/or amend (change) my protected health information that is to be used or disclosed. I have been told in this consent form about any limitations to this right, such as research information that I will not have access to until the end of the study or that will be used strictly for research purposes.

9) My records may also be reviewed by agencies such as the Food and Drug Administration or the National Institutes of Health, or other agencies as required by state or federal regulations.

10) Information about me that may be disclosed includes the following:

- Complete medical record including information regarding diagnosis, illness, treatment, and information that may be recorded about previous diagnosis or treatment. 
- Information gathered as a part of this research study as explained in the informed consent/authorization.

11) I have been told that once my records are disclosed to or used by others, St. Jude Children's Research Hospital cannot guarantee that information will not be further disclosed. Also, the released information may no longer be protected by federal privacy regulations.

12) I have been told that this authorization for the use and disclosure of my protected health information does not expire.

13) I have been told that I may withdraw my authorization for the disclosure or use of my records at anytime, for any reason, with the following exceptions:

- When that information has already been disclosed or used based on my permission

- When the information is required to maintain the integrity of the study

14) To withdraw my authorization, I must complete a Revocation of Release of Authorization form. I have been told I may request this form at St. Jude Children's Research Hospital by calling the Privacy Officer at 901-495-2341. The form must be returned by mail or hand delivery to:

\author{
HIPAA Privacy Officer \\ St. Jude Children's Research Hospital \\ 332 N. Lauderdale \\ Memphis, TN 38105
}

15) I know that if I have more questions about this study or about any injury from the treatment, I can call the Principal Investigator of this study, Dr. Pamela Hinds, at 901/495-3300.

16) I know that I can get more information about my rights as a study participant by calling the Chairman of the Institutional Review Board at 901/495-4357 or the Research Subject Advocate (Ombudsman) at 495-4644. If I am outside of the Memphis area, I may call 1-866583-3472 (1-866-JUDE IRB). This is a toll-free call.

17) I will receive a copy of this signed statement.

18) Telephone contact to remind me of data collection time points. Please initial and date your choice below:

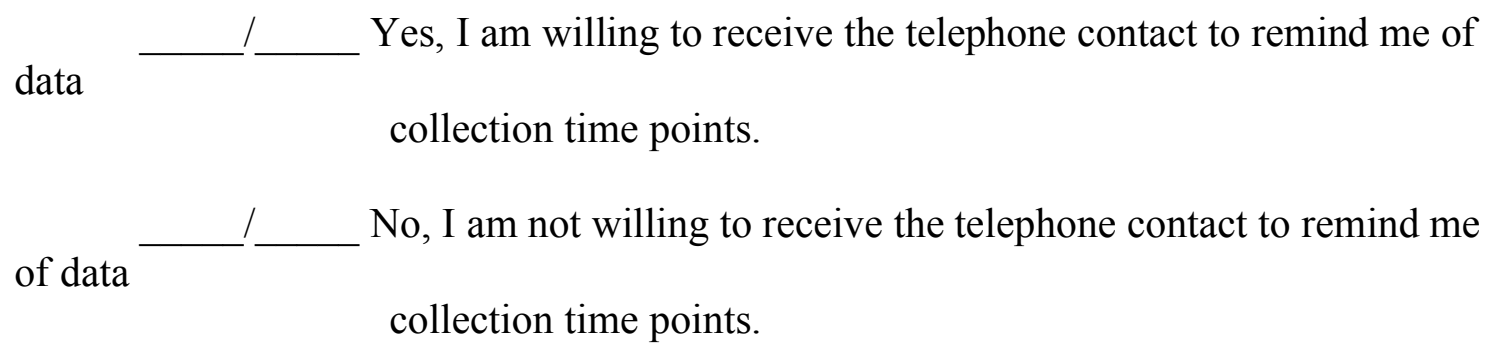

Please fax this page along with the signature page to CPDMO. 


\section{RESEARCH PARTICIPANT STATEMENT}

I have read (or have had read to me) the contents of this document and have been encouraged to ask questions. I have received answers to my questions. I give consent to take part in this research study and authorize the disclosure and use of my child's protected health information for the purposes of that research.

Research Participant

Date

Time (AM/PM)

\section{PHYSICIAN/INVESTIGATOR/DESIGNEE STATEMENT}

I hereby certify that I have discussed the research project with the subject/participant. I have explained all the information contained in the informed consent document, including any risks that may be reasonably expected to occur. I further certify that the research participant was encouraged to ask questions and that all questions were answered.

$\overline{\text { Physician/Investigator/Designee }} \quad \overline{\text { Date }} \quad \overline{\text { Time (AM/PM) }}$

\section{WITNESS STATEMENT}

I have observed the Informed Consent process and certify that the research study, treatment, risks, benefits, and options have been presented to the research participant.

\begin{tabular}{|c|c|c|}
\hline Witness & Date & Time (AM/PM) \\
\hline Interpreter (if needed) & Date & Time (AM/PM) \\
\hline
\end{tabular}

In case of questions or emergencies in reference to this protocol, please contact:

St. Jude Children's Research Hospital

332 North Lauderdale

Memphis, TN 38105

(901) 495-3300 - FAX Interdepartmental \#6265

PLEASE FAX CONSENT FORM TO PROTOCOL OFFICE \#6265 
APPENDIX O

INSTITUTIONAL REVIEW BOARD RESPONSE 


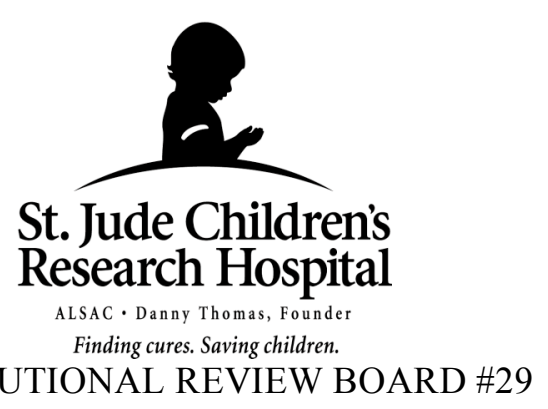

August 11, 2004

Pamela Hinds, RN, PhD

Nursing

Dear Dr. Hinds:

Re: SLEEP3 - Sleep, Fatigue, Caregiver Burden of Parents of Children with ALL

This is to certify on $\mathbf{8 / 1 0 / 0 4}$, the

\section{Response to 7/13/04 IRB review of new protocol; initial version dated 3/25/04, protocol document dated 7/21/04; consent documents dated 07/21/04}

submitted to the Institutional Review Board for consideration was reviewed by the IRB with respect to the study of

human subjects as adequately protecting the rights and welfare of the individuals involved, employing adequate

methods of securing informed consent from these individuals and not involving undue risk in the light of potential

benefits to be derived therefrom.

IRB Review Status:

Approved under 45CFR46.404 and 21CFR50.51 for a period of one year. This approval is effective 8/10/04 through 8/09/05.

Signatory Official

Institutional Review Board

\section{STATEMENT OF PRINCIPAL INVESTIGATOR:}

As previously signed and certified, I understand that approval of this research involving human subjects is contingent upon my agreement:

1. To report to the Institutional Review Board for Human Research (IRB) any adverse effect or research related injuries which might occur in

relation to the human experimentation. I have read and will comply with IRB reporting guidelines.

2. To submit in writing for prior IRB approval any alterations to the plan of human research.

3. To submit timely continuing review reports of this research as requested by the IRB.

4. To maintain copies of all pertinent information related to the research activities in this project, including copies of informed consent 
agreements obtained from all participants.

5. To notify the IRB immediately upon the termination of this project, and/or the departure of the principal investigator from this institution and the project.

\section{CC: CPDMO \\ IRB SLEEP3 File}




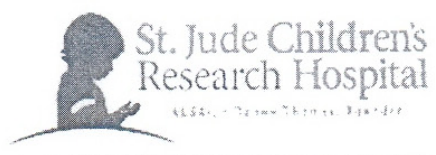

INSTTYUTIONAL REVIEW BOARD \#29

March 28, 2005

Pamela S Hinds, $\mathrm{PhD}, \mathrm{RN}$

Nursing

Dear Dr. Hinds:

RE: SLEEP3 - Sleep, Fatigue, Caregiver Burden of Parents of Children with ALI

This is to certify on March 25, 2005 the

Revision $\$ 1.1$ dated $3 / 9 / 05$ and informed cousent document dated $3 / 9 / 05$

submitted to the Institutional Review Board for consideration was reviewed by the IRB Chair, utilizing expedited review procedures, with respect to the study of human subjects as adequately protecting the rights and welfare of the individuals involved, employing adequate methods of securing informed consent from these individuals and not involving undue risk in the light of potential benefits to be derived therefrom.

IRB Review Status:

Approved as submitted under 45CFR46.110(b)(2) and 21CFR56.110(b)(2) minor modifications to previous approved research.

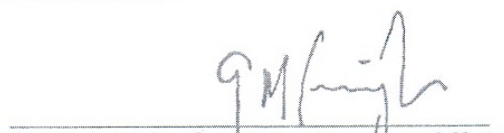

Institutional Review/Board Signawory Official

\section{STATEMENT OF PRINCIPAL INYESTIGATOR:}

As previously signed and certified, I understand that approval of this research involving human subjects is contingent upon my agreement:

1. To report to the Institutional Review Board for Human Research (IRB) any adverse effect or research related injuries which might occur in relation to the human experimentation. I have read and will comply with IRB reporting guidelines.

To submit in writing for prior IRB approval any alterations to the plan of buman research

To submit timely continuing review reports of this research as requested by the IRB.

4. To maintain copies of all pertinent information related to the reseanch activities in this project, including copies of informed consent

agreements obtained from all participants.

To notify the IRB immediately upon the temination of this project, and/or the departure of the principal investigator from this institution and the project.

CC: $\quad$ CPDMO

IRB SLEEP3 File 


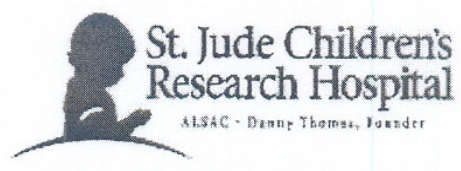

Institutional Review Board \#29

July 26,2006

Pamela S Hinds, PhD, RN

Nursing Research

Dear Dr. Hinds:

RE: SLEEP3 - Sleep, Fatigue, Caregiver Burden of Parents of Children with ALL

This is to certify on $7 / 25 / 2006$ the

Continuing Review Report dated $07 / 10 / 06$, Requesting study be closed to participant accrual, but remain open for follow-up (request study closure to be effective 7/15/06)

submitted to the Institutional Review Board for consideration was reviewed by the IRB with respect to the study of human subjects as adequately protecting the rights and welfare of the individuals involved, employing adequate methods of securing informed consent from these individuals and not involving undue risk in the light of potential benefits to be derived there from.

IRB Review Status:

- Approved under 45CFR46.404 and 21CFR50.51 for a period of one year. This approval is effective 08/10/06 through 08/09/07.

- Study is closed to participant accrual, effective 7/15/06.

- The convened IRB determined that this minimal risk study is eligible for expedited review on its next continuing review under 45 CFR 46.110 (b), category (8)(a).

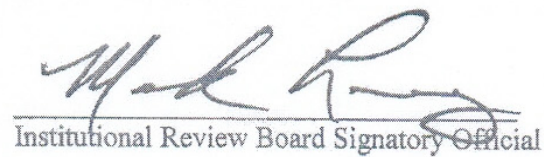

\section{STATEMENT OF PRINCIPAL INVESTIGATOR:}

As previously signed and certified, I understand that approval of this research involving human subjects is contingent upon my agreement:

1. To report to the Institutional Review Board for Human Research (IRB) any adverse effect or research related injuries which might occur in relation to the human experimentation. I have read and will comply with IRB reporting guidelines.

2. To submit in writing for prior IRB approval any alterations to the plan of human research.

3. To submit timely continuing review reports of this research as requested by the IRB.

4. To maintain copies of all pertinent information related to the research activities in this project, including copies of informed consent agreements obtained from all participants.

5. To notify the IRB immediately upon the termination of this project, and/or the departure of the principal investigator from this institution and the project

C: $\quad$ CPDMO

TRB SLEEP3 File

/ms 
April 3, 2007

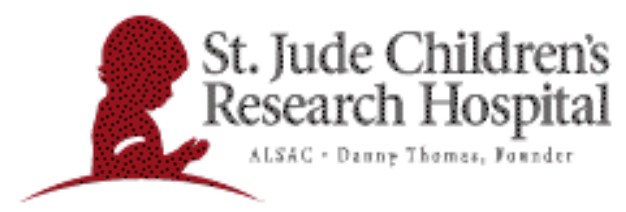

Institutional Review Board \#29

FWA00004775

Pamela S Hinds, PhD, RN

Nursing Research

Dear Dr. Hinds:

RE: SLEEP3 - Sleep, Fatigue, Caregiver Burden of Parents of Children with ALL

This is to certify that, on March 30, 2007, the

Continuing Review Report dated 3/21/07; study to remain closed to participant accrual, but open for follow-up

submitted to the Institutional Review Board for consideration was reviewed by an IRB member using expedited procedures with respect to the adequacy of protecting the rights and welfare of participants, the use of appropriate methods of securing informed consent, the measures to be taken to minimize risk and the degree of risk relative to the potential benefits of the proposed research.

IRB Review Status:

This continuing review report is approved as submitted and the research is re-approved for a period of one year under 45CFR46.110 and 21CFR56.110, Research Category \#8. This study is approved $3 / 30 / 07$ through $3 / 29 / 08$ as proposing human studies, which will be conducted in a manner conforming to ethical principles and regulatory policies of the SJCRH, DHHS and those of the FDA. This study remains closed to patient accrual, but open for follow-up.

Mark Long, MPA, CIP, OHSP Director

\section{STATEMENT OF PRINCIPAL INVESTIGATOR:}

As previously signed and certified, I understand that approval of this research involving human subjects is contingent upon my agreement:

1. To report to the Institutional Review Board for Human Research (IRB) any adverse effect or research related injuries which might occur in relation to the human experimentation. I have read and will comply with IRB reporting guidelines.

2. To submit in writing for prior IRB approval any alterations to the plan of human research.

3. To submit timely continuing review reports of this research as requested by the IRB.

4. To maintain copies of all pertinent information related to the research activities in this project, including copies of informed consent agreements obtained from all participants.

5. To notify the IRB immediately upon the termination of this project, and/or the departure of the principal investigator from this institution and the project.

\section{CC: CPDMO}

IRB SLEEP3 File/cc 


\section{APPENDIX $\mathbf{P}$}

OUTLINE OF PROPOSED TRAINING WORKSHOP 
I. Overview of the Background of the Study
A. Review of Feasibility Study
B. Review of Relevant Literature

II. Overview of the Study Design, Methods, and Instruments
A. Eligibility Criteria
B. Specific enrollment and data collection procedures

\section{Contact CPDMO}

III. Principles of Informed Consent
A. No obligation to participate
B. May withdraw at anytime
C. Honorarium

IV. Confidentiality
A. Promise to parents to be anonymous
B. Research Participants will not be identified
C. Staff

V. Techniques of Interviewing
A. Telephone
1. Inquiring about whether call interrupted parent sleep
B. Face to Face
C. Review of questionnaires
D. Demonstration

1. Permission to tape record, thanks for participating

VI. Techniques for dealing with expression of emotion
A. Reporting adverse events to IRB

VII. Ending Study
A. Expressing gratitude for participation
B. Follow-up Contact 
VIII. Documentation

IX. Data Handling
A. Data Entry
1. Data Bases
B. Actigraph Data
C. Participants' file initiation/storage
D. Transcription process 
APPENDIX Q

SLEEP QUALITY, FATIGUE, AND CAREGIVER BURDEN STATISTICAL RESULTS FOR MOTHERS 
Table Q-1: Descriptive Sleep Quality Results by Actigraph for Mothers ( $n=13)$.

\begin{tabular}{lllllll}
\hline Sleep Variables & Week & Mean & SD & Mdn & Min & Max \\
\hline Efficiency Scores & 1 & 73.85 & 16.12 & 81.29 & 45.01 & 90.52 \\
& 2 & 73.34 & 14.49 & 74.53 & 38.65 & 89.29 \\
Actual Sleep Time & 1 & 332.86 & 89.93 & 335.60 & 182.00 & 466.60 \\
& 2 & 383.04 & 94.47 & 392.75 & 208.67 & 511.50 \\
Sleep Duration & 1 & 475.31 & 51.73 & 472.20 & 369.60 & 547.40 \\
& 2 & 545.66 & 87.93 & 537.80 & 438.60 & 722.00 \\
Nocturnal Awakenings & 1 & 13.92 & 5.30 & 15.20 & 5.00 & 21.75 \\
& & & & & & \\
WASO Scores & 1 & 117.99 & 72.01 & 86.60 & 44.80 & 260.60 \\
& & & & & & \\
& 2 & 134.32 & 75.61 & 134.20 & 48.00 & 334.00 \\
\hline
\end{tabular}


Table Q-2: Difference in Average Sleep Quality Results between Week 1 and Week 2 for Mothers by Actigraph $(n=13)$.

\begin{tabular}{lccccccc}
\hline Sleep Variables & Mean & SD & Mdn & Min & Max & T value & $p$ \\
\hline Efficiency Scores & -0.514 & 9.164 & -1.232 & -11.432 & 17.217 & -0.202 & 0.843 \\
Actual Sleep Time & 50.178 & 81.689 & 34.600 & -25.250 & 224.900 & 2.215 & $0.047^{*}$ \\
Sleep Duration & 70.350 & 80.903 & 77.400 & -82.800 & 201.600 & 3.135 & $0.009^{*}$ \\
Nocturnal Awakenings & 1.588 & 3.978 & 1.800 & -4.350 & 10.000 & 1.440 & 0.176 \\
WASO Scores & 16.329 & 52.045 & 18.200 & -74.550 & 91.800 & 1.131 & 0.280 \\
\hline
\end{tabular}

$* p=<0.05$

Table Q-3: Summary of Longitudinal Proc Mixed Regression Model Findings for Mothers' Actigraphy Scores.

\begin{tabular}{llll}
\hline Response Variable & Covariate & Coefficient & P-value \\
\hline Sleep Efficiency & Week & 0.5 & 0.82 \\
Actual Sleep Time & Week & -51.0 & $0.047^{*}$ \\
Sleep Duration & Week & -70.1 & $0.008^{*}$ \\
Nocturnal Awakenings & Week & -1.6 & 0.21 \\
WASO & Week & -16.7 & 0.23 \\
& & & \\
$* p=<0.05$ & & &
\end{tabular}


Table Q-4: Sleep Duration Results by Actigraph and Sleep Diary Report for Mothers.

\begin{tabular}{|c|c|c|c|c|c|c|c|}
\hline Instrument & Time & $\mathrm{n}$ & Mean & Std & Median & Min & Max \\
\hline \multirow[t]{4}{*}{ Sleep Diary } & Week 1-Day 2 & 12 & 575.42 & 113.41 & 547.50 & 420.00 & 780.00 \\
\hline & Week 1-Day 5 & 11 & 511.82 & 129.25 & 440.00 & 390.00 & 755.00 \\
\hline & Week 2-Day 2 & 12 & 501.67 & 103.84 & 472.50 & 375.00 & 750.00 \\
\hline & Week 2-Day 5 & 8 & 487.50 & 79.64 & 497.50 & 375.00 & 575.00 \\
\hline \multirow[t]{4}{*}{ Actigraph } & Week 1-Day 2 & 12 & 458.83 & 161.67 & 469.00 & 47.00 & 631.00 \\
\hline & Week 1-Day 5 & 11 & 414.73 & 137.17 & 363.00 & 246.00 & 679.00 \\
\hline & Week 2-Day 2 & 12 & 495.33 & 117.38 & 513.50 & 299.00 & 675.00 \\
\hline & Week 2-Day 5 & 8 & 501.88 & 159.00 & 469.50 & 288.00 & 721.00 \\
\hline
\end{tabular}


Table Q-5: Sleep Quality Results for Matched Mother and Child by Actigraphy $(n=12)$.

\begin{tabular}{|c|c|c|c|c|c|c|c|}
\hline Sleep Variables & Parent/Child & Week & Mean & SD & Mdn & Min & Max \\
\hline \multirow[t]{4}{*}{ Efficiency Scores } & Mother & 1 & 77.87 & 14.03 & 85.29 & 45.01 & 90.52 \\
\hline & & 2 & 73.01 & 15.46 & 73.55 & 38.65 & 89.29 \\
\hline & Child & 1 & 82.78 & 12.06 & 85.52 & 68.39 & 98.26 \\
\hline & & 2 & 82.53 & 11.87 & 85.25 & 56.47 & 96.97 \\
\hline \multirow[t]{4}{*}{ Actual Sleep } & Mother & 1 & 350.51 & 82.70 & 373.60 & 205.00 & 466.60 \\
\hline & & 2 & 364.88 & 93.02 & 376.93 & 208.67 & 493.20 \\
\hline & Child & 1 & 399.93 & 98.15 & 435.00 & 229.40 & 533.60 \\
\hline & & 2 & 435.42 & 148.30 & 440.00 & 120.00 & 641.40 \\
\hline \multirow[t]{4}{*}{ Sleep Duration } & Mother & 1 & 474.09 & 54.28 & 475.80 & 369.60 & 547.40 \\
\hline & & 2 & 528.31 & 73.16 & 529.20 & 432.00 & 672.20 \\
\hline & Child & 1 & 500.93 & 45.69 & 502.80 & 401.60 & 553.00 \\
\hline & & 2 & 544.05 & 102.38 & 532.00 & 333.00 & 688.60 \\
\hline \multirow[t]{4}{*}{ Nocturnal Awakenings } & Mother & 1 & 14.26 & 4.72 & 16.00 & 5.00 & 19.40 \\
\hline & & 2 & 15.91 & 6.00 & 15.61 & 5.50 & 25.00 \\
\hline & Child & 1 & 16.80 & 8.18 & 16.90 & 3.80 & 26.40 \\
\hline & & 2 & 13.69 & 6.49 & 11.70 & 5.25 & 23.80 \\
\hline \multirow[t]{4}{*}{ WASO Scores } & Mother & 1 & 101.55 & 65.82 & 71.80 & 44.80 & 260.60 \\
\hline & & 2 & 134.89 & 78.53 & 146.20 & 48.00 & 334.00 \\
\hline & Child & 1 & 79.16 & 49.39 & 72.40 & 19.40 & 149.20 \\
\hline & & 2 & 90.32 & 54.17 & 89.67 & 24.00 & 226.40 \\
\hline
\end{tabular}


Table Q-6: Summary of Proc Mixed Model Regression Findings for Matched Mother and Child.

\begin{tabular}{llll}
\hline $\begin{array}{l}\text { Mother } \\
\text { Response Variable }\end{array}$ & Covariate & & \\
\hline Mother Sleep Efficiency & Chdependent Variable & Coefficient & P-value \\
& Week & 0.02 & 0.84 \\
Mother Actual Sleep & Child Actual Sleep Time & 0.35 & 0.47 \\
Time & Week & -40.3 & 0.12 \\
Mother Sleep Duration & Child Sleep Duration & 0.43 & $0.0001^{*}$ \\
& Week & -63.9 & $0.017^{*}$ \\
Mother Nocturnal & Child Nocturnal & 0.18 & 0.053 \\
Awakenings & Awakenings Week & -2.18 & 0.096 \\
Mother WASO & Child WASO & -0.013 & 0.92 \\
& Week & -25.7 & 0.095 \\
\hline$* p=<0.05$ & & &
\end{tabular}

Table Q-7: Descriptive Brief Fatigue Inventory Results for Mothers.

\begin{tabular}{lcccccc}
\hline Variables & $\mathrm{n}$ & Mean & SD & Mdn & Min & Max \\
\hline Week 1 Day 2 & 15 & 2.87 & 1.75 & 2.67 & 0.44 & 5.56 \\
Week 1 Day 5 & 15 & 2.51 & 1.73 & 2.67 & 0.00 & 6.89 \\
Week 2 Day 2 & 13 & 2.98 & 1.44 & 2.78 & 1.00 & 5.11 \\
Week 2 Day 5 & 14 & 3.37 & 1.78 & 3.17 & 1.00 & 7.67 \\
\hline
\end{tabular}


Table Q-8: Differences in Average Brief Fatigue Inventory Results between Week 1 and Week 2 for Mothers.

\begin{tabular}{|c|c|c|c|c|c|c|c|c|}
\hline Difference of Average & $\mathrm{n}$ & Mean & SD & Mdn & Min & Max & $t$ & $p$ \\
\hline Day 2 (Week $2-$ Week 1$)$ & 13 & 0.26 & 1.03 & 0.44 & -1.11 & 1.78 & 0.93 & 0.37 \\
\hline Day 5 (Week $2-$ Week 1) & 14 & 0.98 & 1.36 & 0.50 & -0.11 & 4.11 & 2.70 & $0.02 *$ \\
\hline Week 2 - Week 1 & 13 & 0.50 & 0.92 & 0.22 & -0.56 & 2.56 & 1.98 & 0.07 \\
\hline
\end{tabular}

Table Q-9: Descriptive Revised Informal Caregiver Survey Results for Mothers.

\begin{tabular}{lccccccc}
\hline Week & Mean & Std & Mdn & Min & Max & $t$ & $p$ \\
\hline Week 1 & 13.07 & 6.35 & 14.00 & 1.00 & 21.00 & & \\
Week 2 & 15.50 & 7.56 & 16.00 & 4.00 & 27.00 & & \\
Difference of Average & 2.43 & 4.15 & 2.50 & -5.00 & 11.00 & 2.19 & 0.05 \\
\hline
\end{tabular}


Table Q-10: Descriptive Care of My Child with Cancer Instrument Results for Mothers $(n=12)$.

\begin{tabular}{|c|c|c|c|c|c|c|}
\hline Domain & Week & Mean & SD & Mdn & Min & Max \\
\hline \multirow[t]{2}{*}{ Physical Care (13 items) } & 1 & 18.98 & 7.51 & 17.28 & 9.00 & 40.08 \\
\hline & 2 & 19.12 & 6.38 & 17.93 & 10.00 & 34.93 \\
\hline \multirow[t]{2}{*}{ Emotional Care (7 Items) } & 1 & 12.82 & 4.15 & 12.32 & 3.00 & 22.81 \\
\hline & 2 & 15.04 & 5.29 & 14.39 & 10.02 & 29.91 \\
\hline \multirow[t]{2}{*}{ Finances (1 item) } & 1 & 1.53 & 0.48 & 1.41 & 1.00 & 2.00 \\
\hline & 2 & 1.59 & 0.48 & 1.41 & 1.00 & 2.45 \\
\hline \multirow{3}{*}{$\begin{array}{l}\text { Family Relationships } \\
\text { (4 items) }\end{array}$} & 1 & 9.94 & 2.46 & 10.11 & 6.66 & 15.22 \\
\hline & & & & & & \\
\hline & 2 & 10.68 & 3.77 & 10.51 & 1.00 & 17.46 \\
\hline \multirow[t]{2}{*}{ Communication (2 items) } & 1 & 2.87 & 1.03 & 2.83 & 1.73 & 5.46 \\
\hline & 2 & 3.21 & 1.31 & 3.22 & 1.73 & 5.91 \\
\hline \multirow[t]{2}{*}{ Education (1 item) } & 1 & 1.75 & 0.96 & 1.41 & 1.00 & 4.00 \\
\hline & 2 & 2.14 & 1.29 & 2.00 & 1.00 & 5.00 \\
\hline
\end{tabular}


Table Q-11: Differences in Care of My Child with Cancer Instrument Results between Week 1 and Week 2 for Mothers.

\begin{tabular}{lccccccc}
\hline Domain & Mean & SD & Mdn & Min & Max & $t$ & $p$ \\
\hline Physical Care (13 items) & 1.77 & 6.73 & 0.18 & -9.54 & 18.52 & 0.95 & 0.36 \\
Emotional Care (7 Items) & 0.54 & 6.45 & 0.00 & -11.11 & 17.40 & 0.30 & 0.77 \\
Finances (1 item) & 0.11 & 0.47 & 0.00 & -0.59 & 1.45 & 0.78 & 0.45 \\
Family Relationships & 1.55 & 3.35 & 1.35 & -2.60 & 10.58 & 1.60 & 0.14 \\
$\begin{array}{l}\text { (4 items) } \\
\text { Communication (2 items) }\end{array}$ & 0.35 & 1.46 & 0.00 & -1.46 & 3.91 & 0.84 & 0.42 \\
Education (1 item) & 0.32 & 0.86 & 0.00 & -0.54 & 2.55 & 1.30 & 0.22 \\
\hline
\end{tabular}


APPENDIX R

SLEEP QUALITY, FATIGUE, AND CAREGIVER BURDEN STATISTICAL RESULTS FOR FATHERS 
Table R-1: Descriptive Sleep Quality Results by Actigraphy for Fathers $(n=5)$.

\begin{tabular}{lllllll}
\hline Sleep Variables & Week & Mean & SD & Mdn & Min & Max \\
\hline Efficiency Scores & 1 & 63.31 & 20.95 & 56.91 & 43.27 & 85.99 \\
& 2 & 75.74 & 19.02 & 87.21 & 54.85 & 92.22 \\
Actual Sleep Time & 1 & 281.10 & 87.60 & 286.50 & 188.80 & 384.40 \\
& 2 & 342.87 & 122.69 & 350.00 & 193.75 & 528.00 \\
Sleep Duration & 1 & 504.44 & 67.19 & 482.80 & 418.20 & 592.80 \\
& 2 & 561.23 & 182.66 & 514.50 & 422.40 & 866.67 \\
Nocturnal Awakenings & 1 & 14.13 & 3.84 & 15.20 & 9.20 & 18.60 \\
& 2 & 10.06 & 4.89 & 12.00 & 1.33 & 12.75 \\
WASO Scores & 1 & 160.99 & 90.70 & 198.75 & 63.20 & 244.40 \\
& 2 & 111.69 & 78.88 & 81.33 & 43.60 & 228.80 \\
\hline
\end{tabular}


Table R-2: Sleep Duration Results by Actigraph and Sleep Diary Report for Fathers $(n=5)$.

\begin{tabular}{lllllll}
\hline Instrument & Time & Mean & Std & Mdn & Min & Max \\
& Week 1-Day 2 & 462.14 & 51.55 & 480.00 & 360.00 & 510.00 \\
Sleep Diary & Week 1-Day 5 & 400.83 & 50.83 & 402.50 & 315.00 & 465.00 \\
& Week 2-Day 2 & 416.67 & 74.81 & 430.00 & 305.00 & 495.00 \\
& Week 2-Day 5 & 420.00 & 51.96 & 435.00 & 330.00 & 465.00 \\
& Week 1-Day 2 & 517.40 & 62.31 & 527.00 & 443.00 & 588.00 \\
Actigraph & Week 1-Day 5 & 488.25 & 145.81 & 454.50 & 350.00 & 694.00 \\
& Week 2-Day 2 & 540.40 & 185.45 & 444.00 & 372.00 & 820.00 \\
& Week 2-Day 5 & 494.33 & 129.09 & 475.00 & 376.00 & 632.00 \\
\hline
\end{tabular}


Table R-3: Difference in Sleep Duration Results between Actigraph and Sleep Diary for Fathers $(\mathrm{n}=5)$.

\begin{tabular}{lccccc}
\hline $\begin{array}{l}\text { Sleep Diary - } \\
\text { Actigraph }\end{array}$ & Mean & SD & Mdn & \multicolumn{1}{l}{ Min } & Max \\
\hline Week 1-Day 2 & 29.40 & 55.86 & 17.00 & -37.00 & 108.00 \\
Week 1-Day 5 & 74.50 & 175.05 & 52.00 & -115.00 & 309.00 \\
Week 2-Day 2 & 101.40 & 158.11 & 84.00 & -88.00 & 340.00 \\
Week 2-Day 5 & 204.33 & 267.08 & 197.00 & -59.00 & 475.00 \\
\hline
\end{tabular}


Table R-4: Sleep Quality Results for Matched Father and Child by Actigraphy ( $\mathrm{n}=4)$.

\begin{tabular}{|c|c|c|c|c|c|c|c|}
\hline Sleep Variables & Parent/Child & Week & Mean & SD & Mdn & Min & Max \\
\hline Efficiency & Father & 1 & 59.60 & 17.56 & 53.61 & 46.08 & 85.08 \\
\hline \multirow[t]{3}{*}{ Scores } & & 2 & 81.71 & 15.73 & 89.28 & 63.63 & 92.22 \\
\hline & Child & 1 & 71.63 & 27.21 & 81.15 & 40.93 & 92.80 \\
\hline & & 2 & 65.67 & 25.07 & 65.67 & 47.94 & 83.39 \\
\hline \multirow[t]{4}{*}{ Actual Sleep } & Father & 1 & 265.77 & 67.46 & 262.10 & 190.67 & 348.20 \\
\hline & & 2 & 423.84 & 91.22 & 385.33 & 358.20 & 528.00 \\
\hline & Child & 1 & 343.95 & 169.85 & 399.00 & 153.40 & 479.44 \\
\hline & & 2 & 369.10 & 161.08 & 369.10 & 255.20 & 483.00 \\
\hline \multirow[t]{4}{*}{ Sleep Duration } & Father & 1 & 499.03 & 57.15 & 515.45 & 418.20 & 547.00 \\
\hline & & 2 & 643.54 & 222.14 & 641.56 & 422.40 & 866.67 \\
\hline & Child & 1 & 490.20 & 52.58 & 513.50 & 430.00 & 527.11 \\
\hline & & 2 & 587.20 & 11.03 & 587.20 & 579.40 & 595.00 \\
\hline Nocturnal & Father & 1 & 13.23 & 4.92 & 13.46 & 7.40 & 18.60 \\
\hline \multirow[t]{3}{*}{ Awakenings } & & 2 & 7.56 & 5.55 & 9.33 & 1.33 & 12.00 \\
\hline & Child & 1 & 12.07 & 4.23 & 14.00 & 7.22 & 15.00 \\
\hline & & 2 & 16.77 & 5.04 & 16.77 & 13.20 & 20.33 \\
\hline \multirow[t]{4}{*}{ WASO Scores } & Father & 1 & 176.96 & 77.67 & 203.33 & 63.20 & 238.00 \\
\hline & & 2 & 99.76 & 67.29 & 81.33 & 43.60 & 174.33 \\
\hline & Child & 1 & 129.27 & 105.50 & 91.75 & 47.67 & 248.40 \\
\hline & & 2 & 180.27 & 117.29 & 180.27 & 97.33 & 263.20 \\
\hline
\end{tabular}


Table R-5: Descriptive Brief Fatigue Inventory Results for Fathers.

\begin{tabular}{lcccccc}
\hline Variables & $\mathrm{n}$ & Mean & SD & Mdn & Min & Max \\
\hline Week 1 Day 2 & 6 & 4.35 & 0.59 & 4.17 & 3.78 & 5.33 \\
Week 1 Day 5 & 5 & 2.91 & 0.64 & 2.78 & 2.33 & 4.00 \\
Week 2 Day 2 & 6 & 3.43 & 2.10 & 3.78 & 0.00 & 6.00 \\
Week 2 Day 5 & 6 & 2.98 & 2.16 & 2.00 & 1.00 & 5.89 \\
\hline
\end{tabular}

Table R-6: Descriptive Revised Informal Caregiver Survey Results for Fathers.

\begin{tabular}{lcccccc}
\hline Week & $\mathrm{n}$ & Mean & SD & Mdn & Min & Max \\
\hline Week 1 & 7 & 11.00 & 3.74 & 10.00 & 7.00 & 19.00 \\
Week 2 & 6 & 12.50 & 7.82 & 10.00 & 7.00 & 28.00 \\
\hline
\end{tabular}


Table R-7: Descriptive Care of My Child with Cancer Instrument Results for Fathers $(n=5)$.

\begin{tabular}{|c|c|c|c|c|c|c|}
\hline Domain & Week & Mean & SD & Mdn & Min & Max \\
\hline \multirow[t]{2}{*}{ Physical Care (13 items) } & 1 & 18.79 & 4.60 & 17.81 & 13.00 & 25.35 \\
\hline & 2 & 16.17 & 4.67 & 15.02 & 11.00 & 23.00 \\
\hline \multirow[t]{2}{*}{ Emotional Care (7 Items) } & 1 & 14.69 & 2.45 & 14.12 & 12.24 & 18.02 \\
\hline & 2 & 12.65 & 7.72 & 12.41 & 1.00 & 24.91 \\
\hline \multirow[t]{2}{*}{ Finances (1 item) } & 1 & 1.53 & 0.90 & 1.00 & 1.00 & 3.16 \\
\hline & 2 & 1.07 & 0.17 & 1.00 & 1.00 & 1.41 \\
\hline \multirow{3}{*}{$\begin{array}{l}\text { Family Relationships } \\
\text { (4 items) }\end{array}$} & 1 & 9.67 & 3.18 & 9.08 & 6.16 & 13.93 \\
\hline & & & & & & \\
\hline & 2 & 9.01 & 4.65 & 6.90 & 5.45 & 17.00 \\
\hline \multirow[t]{2}{*}{ Communication (2 items) } & 1 & 3.55 & 1.94 & 2.93 & 2.00 & 7.00 \\
\hline & 2 & 3.34 & 1.03 & 3.00 & 2.00 & 4.69 \\
\hline \multirow[t]{2}{*}{ Education (1 item) } & 1 & 2.05 & 0.38 & 2.00 & 1.41 & 2.45 \\
\hline & 2 & 2.32 & 0.73 & 2.45 & 1.41 & 3.00 \\
\hline
\end{tabular}




\section{APPENDIX S}

SLEEP QUALITY, FATIGUE, AND CAREGIVER BURDEN STATISTICAL RESULTS FOR COUPLES 
Table S-1: Descriptive Sleep Quality Results by Actigraphy for Couples ( $\mathrm{n}=4)$.

\begin{tabular}{|c|c|c|c|c|c|c|c|}
\hline Sleep Variables & Week & Parent & Mean & SD & Mdn & Min & Max \\
\hline \multicolumn{8}{|c|}{ Efficiency Scores } \\
\hline & 1 & Mother & 69.84 & 20.94 & 70.89 & 47.04 & 90.52 \\
\hline & & Father & 58.19 & 24.10 & 45.31 & 43.27 & 85.99 \\
\hline & 2 & Mother & 78.15 & 11.51 & 79.87 & 63.59 & 89.29 \\
\hline & & Father & 65.73 & 18.61 & 55.13 & 54.85 & 87.21 \\
\hline \multicolumn{8}{|c|}{ Actual Sleep Time } \\
\hline & 1 & Mother & 331.24 & 123.84 & 338.18 & 182.00 & 466.60 \\
\hline & & Father & 256.93 & 110.48 & 197.60 & 188.80 & 384.40 \\
\hline & 2 & Mother & 426.58 & 68.43 & 412.40 & 370.00 & 511.50 \\
\hline & & Father & 276.05 & 78.46 & 284.40 & 193.75 & 350.00 \\
\hline \multicolumn{8}{|l|}{ Sleep Duration } \\
\hline & 1 & Mother & 490.51 & 35.79 & 494.58 & 451.50 & 521.40 \\
\hline & & Father & 519.00 & 63.92 & 482.80 & 481.40 & 592.80 \\
\hline & 2 & Mother & 567.45 & 117.45 & 554.60 & 438.60 & 722.00 \\
\hline & & Father & 505.70 & 76.28 & 514.50 & 425.40 & 577.20 \\
\hline \multicolumn{8}{|c|}{ Nocturnal Awakenings } \\
\hline & 1 & Mother & 11.78 & 8.01 & 10.18 & 5.00 & 21.75 \\
\hline & & Father & 13.60 & 3.86 & 15.20 & 9.20 & 16.40 \\
\hline & 2 & Mother & 11.85 & 6.30 & 12.00 & 6.00 & 17.40 \\
\hline & & Father & 12.32 & 0.39 & 12.20 & 12.00 & 12.75 \\
\hline \multicolumn{8}{|l|}{ WASO Scores } \\
\hline & 1 & Mother & 135.03 & 84.09 & 127.65 & 54.80 & 230.00 \\
\hline & & Father & 181.00 & 101.78 & 235.00 & 63.60 & 244.40 \\
\hline & 2 & Mother & 103.76 & 57.59 & 86.33 & 58.20 & 184.20 \\
\hline & & Father & 144.50 & 89.72 & 154.50 & 50.20 & 228.80 \\
\hline
\end{tabular}


Table S-2: Sleep Duration Results by Actigraph and Sleep Diary Report for Couples $(\mathrm{n}=4)$.

\begin{tabular}{|c|c|c|c|c|c|c|c|}
\hline Instrument & Time & Parent & Mean & SD & Mdn & Min & Max \\
\hline \multicolumn{8}{|l|}{ Sleep Diary } \\
\hline & Week 1 Day 2 & Mother & 618.33 & 85.05 & 615.00 & 535.00 & 705.00 \\
\hline & & Father & 498.33 & 16.07 & 505.00 & 480.00 & 510.00 \\
\hline & Week 1 Day 5 & Mother & 435.00 & 14.57 & 423.00 & 405.00 & 435.00 \\
\hline & & Father & 396.67 & 10.41 & 400.00 & 385.00 & 405.00 \\
\hline & Week 2 Day 2 & Mother & 470.00 & 100.42 & 447.50 & 375.00 & 610.00 \\
\hline & & Father & 418.33 & 69.34 & 400.00 & 360.00 & 495.00 \\
\hline & Week 2 Day 5 & Mother & 475.00 & 141.42 & 475.00 & 375.00 & 575.00 \\
\hline & & Father & 435.00 & 52.01 & 415.00 & 335.00 & 435.00 \\
\hline \multicolumn{8}{|l|}{ Actigraph } \\
\hline & Week 1 Day 2 & Mother & 459.00 & 87.49 & 486.00 & 332.00 & 532.00 \\
\hline & & Father & 511.33 & 62.00 & 527.00 & 443.00 & 564.00 \\
\hline & Week 1 Day 5 & Mother & 507.67 & 148.54 & 429.00 & 415.00 & 679.00 \\
\hline & & Father & 534.33 & 138.38 & 460.00 & 449.00 & 694.00 \\
\hline & Week 2 Day 2 & Mother & 531.25 & 86.31 & 513.50 & 448.00 & 650.00 \\
\hline & & Father & 503.33 & 116.00 & 444.00 & 429.00 & 637.00 \\
\hline & Week 2 Day 5 & Mother & 479.67 & 209.86 & 378.00 & 340.00 & 721.00 \\
\hline & & Father & 504.00 & 181.02 & 504.00 & 376.00 & 632.00 \\
\hline
\end{tabular}


Table S-3: Sleep Quality Results for Matched Couple and Child ( $\mathrm{n}=4)$.

\begin{tabular}{|c|c|c|c|c|c|c|c|}
\hline Sleep Variables & Week & $\begin{array}{l}\text { Parent/ } \\
\text { Child }\end{array}$ & Mean & SD & Mdn & Min & Max \\
\hline \multirow[t]{6}{*}{ Efficiency Scores } & 1 & Mother & 69.84 & 20.94 & 70.89 & 47.04 & 90.52 \\
\hline & \multirow[t]{2}{*}{1} & Father & 58.19 & 24.10 & 45.31 & 43.27 & 85.99 \\
\hline & & Child & 93.19 & 0.55 & 93.19 & 92.80 & 93.58 \\
\hline & 2 & Mother & 78.15 & 11.51 & 79.87 & 63.59 & 89.29 \\
\hline & \multirow[t]{2}{*}{2} & Father & 65.73 & 18.61 & 55.13 & 54.85 & 87.21 \\
\hline & & Child & 86.66 & 4.83 & 88.52 & 81.18 & 90.28 \\
\hline \multirow[t]{6}{*}{ Actual Sleep Time } & 1 & Mother & 331.24 & 123.84 & 338.18 & 182.00 & 466.60 \\
\hline & \multirow[t]{2}{*}{1} & Father & 256.93 & 110.48 & 197.60 & 188.80 & 384.40 \\
\hline & & Child & 464.82 & 20.68 & 464.82 & 450.20 & 479.44 \\
\hline & 2 & Mother & 426.58 & 68.43 & 412.40 & 370.00 & 511.50 \\
\hline & \multirow[t]{2}{*}{2} & Father & 276.05 & 78.46 & 284.40 & 193.75 & 350.00 \\
\hline & & Child & 464.84 & 76.31 & 430.20 & 412.00 & 552.33 \\
\hline \multirow[t]{6}{*}{ Sleep Duration } & 1 & Mother & 490.51 & 35.79 & 494.58 & 451.50 & 521.40 \\
\hline & \multirow[t]{2}{*}{1} & Father & 519.00 & 63.92 & 482.80 & 481.40 & 592.80 \\
\hline & & Child & 505.36 & 30.77 & 505.36 & 483.60 & 527.11 \\
\hline & 2 & Mother & 567.45 & 117.45 & 554.60 & 438.60 & 722.00 \\
\hline & \multirow[t]{2}{*}{2} & Father & 505.70 & 76.28 & 514.50 & 425.40 & 577.20 \\
\hline & & Child & 543.93 & 83.53 & 532.00 & 467.00 & 632.78 \\
\hline \multirow{6}{*}{$\begin{array}{l}\text { Nocturnal } \\
\text { Awakenings }\end{array}$} & 1 & Mother & 11.78 & 8.01 & 10.18 & 5.00 & 21.75 \\
\hline & \multirow[t]{2}{*}{1} & Father & 13.60 & 3.86 & 15.20 & 9.20 & 16.40 \\
\hline & & Child & 11.54 & 4.14 & 12.07 & 6.80 & 15.22 \\
\hline & 2 & Mother & 11.85 & 6.30 & 12.00 & 6.00 & 17.40 \\
\hline & \multirow[t]{2}{*}{2} & Father & 12.32 & 0.39 & 12.20 & 12.00 & 12.75 \\
\hline & & Child & 12.17 & 0.24 & 12.17 & 12.00 & 12.33 \\
\hline \multirow[t]{6}{*}{ WASO Scores } & 1 & Mother & 135.03 & 84.09 & 127.65 & 54.80 & 230.00 \\
\hline & \multirow[t]{2}{*}{1} & Father & 181.00 & 101.78 & 235.00 & 63.60 & 244.40 \\
\hline & & Child & 39.13 & 12.07 & 39.13 & 30.60 & 47.67 \\
\hline & 2 & Mother & 103.76 & 57.59 & 86.33 & 58.20 & 184.20 \\
\hline & \multirow[t]{2}{*}{2} & Father & 144.50 & 89.72 & 154.50 & 50.20 & 228.80 \\
\hline & & Child & 75.21 & 29.55 & 80.44 & 43.40 & 101.80 \\
\hline
\end{tabular}


Table S-4: Descriptive Brief Fatigue Inventory Results for Couples $(n=4)$.

\begin{tabular}{lllllll}
\hline Variables & Parent & Mean & SD & Mdn & Min & Max \\
\hline Week 1 Day 2 & & & & & & \\
& Mother & 3.33 & 2.06 & 3.67 & 0.78 & 5.22 \\
& Father & 3.89 & 1.83 & 4.56 & 1.33 & 5.56 \\
Week 1 Day 5 & & & & & & \\
& Mother & 2.98 & 2.25 & 2.33 & 1.33 & 6.89 \\
Week 2 Day 2 & Father & 3.09 & 1.34 & 2.78 & 1.22 & 4.56 \\
& & & & & & \\
Meek 2 Day 5 & Mother & 2.92 & 1.53 & 2.61 & 1.44 & 5.00 \\
& Father & 3.17 & 1.69 & 3.11 & 1.56 & 4.89 \\
& & & & & & \\
& Mother & 3.87 & 2.73 & 2.67 & 1.44 & 7.67 \\
\hline
\end{tabular}

Table S-5: Descriptive Revised Informal Caregiver Survey Results for Couples (n=4).

\begin{tabular}{lllllll}
\hline Week & Parent & Mean & SD & Mdn & Min & Max \\
\hline Week 1 & & & & & & \\
& Mother & 10.20 & 7.26 & 9.00 & 1.00 & 21.00 \\
& Father & 11.40 & 4.51 & 10.00 & 7.00 & 19.00 \\
Week 2 & & & & & & \\
& Mother & 15.00 & 9.03 & 15.00 & 4.00 & 27.00 \\
& Father & 13.75 & 9.64 & 10.00 & 7.00 & 28.00 \\
Difference of Average & & & & & & \\
& Mother & 4.80 & 3.90 & 3.00 & 1.00 & 11.00 \\
& Father & 2.25 & 4.57 & 0.50 & -1.00 & 9.00 \\
\hline
\end{tabular}


Table S-6: Descriptive Care of My Child with Cancer Results for Couples ( $\mathrm{n}=4)$.

\begin{tabular}{|c|c|c|c|c|c|c|c|}
\hline Domain & Week & Parent & Mean & SD & Mdn & Min & Max \\
\hline \multicolumn{8}{|c|}{ Physical Care } \\
\hline & Week 1 & Mother & 21.11 & 10.78 & 17.45 & 13.00 & 40.08 \\
\hline & & Father & 19.40 & 4.86 & 18.16 & 13.00 & 25.35 \\
\hline & Week 2 & Mother & 16.31 & 2.24 & 17.16 & 13.00 & 17.93 \\
\hline & & Father & 17.25 & 5.06 & 16.50 & 13.00 & 23.00 \\
\hline \multicolumn{8}{|c|}{ Emotional Care } \\
\hline & Week 1 & Mother & 11.37 & 4.19 & 12.72 & 3.00 & 14.11 \\
\hline & & Father & 15.08 & 2.53 & 15.46 & 12.24 & 18.02 \\
\hline & Week 2 & Mother & 13.32 & 2.06 & 13.45 & 11.14 & 15.24 \\
\hline & & Father & 15.61 & 6.51 & 13.67 & 10.18 & 24.91 \\
\hline \multicolumn{8}{|l|}{ Finances } \\
\hline & Week 1 & Mother & 1.48 & 0.50 & 1.41 & 1.00 & 2.00 \\
\hline & & Father & 1.63 & 0.96 & 1.00 & 1.00 & 3.16 \\
\hline & Week 2 & Mother & 1.21 & 0.24 & 1.21 & 1.00 & 1.41 \\
\hline & & Father & 1.00 & 0.00 & 1.00 & 1.00 & 1.00 \\
\hline \multicolumn{8}{|c|}{ Family Relationships } \\
\hline & Week 1 & Mother & 9.11 & 1.51 & 9.35 & 7.46 & 10.93 \\
\hline & & Father & 10.21 & 3.23 & 10.16 & 6.16 & 13.93 \\
\hline & Week 2 & Mother & 9.94 & 0.93 & 9.92 & 9.00 & 10.93 \\
\hline & & Father & 9.59 & 5.15 & 7.95 & 5.45 & 17.00 \\
\hline \multicolumn{8}{|c|}{ Communication } \\
\hline & Week 1 & Mother & 2.57 & 0.52 & 2.83 & 2.00 & 3.00 \\
\hline & & Father & 3.58 & 2.17 & 2.41 & 2.00 & 7.00 \\
\hline & Week 2 & Mother & 2.86 & 1.16 & 2.50 & 2.00 & 4.45 \\
\hline & & Father & 3.67 & 0.82 & 3.50 & 3.00 & 4.69 \\
\hline \multicolumn{8}{|c|}{ Education } \\
\hline & Week 1 & Mother & 1.28 & 0.44 & 1.00 & 1.00 & 2.00 \\
\hline & & Father & 2.18 & 0.25 & 2.00 & 2.00 & 2.45 \\
\hline & Week 2 & Mother & 1.75 & 0.96 & 1.50 & 1.00 & 3.00 \\
\hline & & Father & 2.55 & 0.60 & 2.72 & 1.73 & 3.00 \\
\hline \multicolumn{8}{|c|}{ CMCC Total Score } \\
\hline & Week 1 & Mother & 48.60 & 12.53 & 43.93 & 38.16 & 70.29 \\
\hline & & Father & 52.07 & 10.11 & 53.93 & 37.80 & 63.33 \\
\hline & Week 2 & Mother & 45.39 & 4.06 & 44.97 & 41.24 & 50.37 \\
\hline & & Father & 49.66 & 16.54 & 46.85 & 34.36 & 70.60 \\
\hline
\end{tabular}




\section{VITA}

Jerithea Doronice Tidwell was born in San Diego, California, on June 5, 1972. She was awarded a Bachelor of Science in Nursing from the University of Arkansas, Pine Bluff in 1995. She attended the University of Nebraska Medical Center where she received her Master of Science in Nursing in Pediatrics in 1999. She has worked in the area of maternal child and pediatric nursing for over 10 years.

She began her career in Pine Bluff, Arkansas as a post-partum nurse at Jefferson Regional Medical Center. She later worked at Omaha Children's Hospital on the medical surgical and neonatal step down units, at University Medical Center on the pediatric liver bowel transplant unit and at Union University in Lincoln Nebraska as a clinical instructor. After her graduate education, she served as the Leukemia Clinical Nurse Specialist, Hematology/Oncology Unit Educator and Bone Marrow Transplant Staff nurse at St. Jude Children's Research Hospital in Memphis TN. She also worked as an assistant professor at Union University at Germantown, TN, where she instructed graduate and undergraduate students and helped develop the Pediatric Advance Practice Program. Jerithea was certified as a Pediatric Clinical Nurse Specialist and Pediatric Nurse Practitioner by ANCC in 1995 and 1996, respectively. She has presented on various pediatric topics at several national conferences. In 2007 she served as a test reviewer for the ANCC Pediatric Clinical Nurse Specialist Certification Exam.

Jerithea Tidwell received a Doctor of Philosophy in nursing degree from the University of Tennessee Health Science Center in May, 2008. She remains employed at St Jude Children's Research Hospital. 